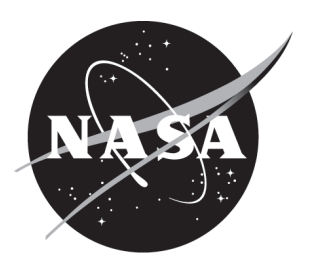

\title{
Three-Dimensional Numerical Modeling of Magnetohydrodynamic Augmented Propulsion Experiment
}

M.W. Turner and C.W. Hawk

The University of Alabama in Huntsville, Huntsville, Alabama

R.J. Litchford

Marshall Space Flight Center, Marshall Space Flight Center, Alabama 


\section{The NASA STI Program....in Profile}

Since its founding, NASA has been dedicated to the advancement of aeronautics and space science. The NASA Scientific and Technical Information (STI) Program Office plays a key part in helping NASA maintain this important role.

The NASA STI program operates under the auspices of the Agency Chief Information Officer. It collects, organizes, provides for archiving, and disseminates NASA's STI. The NASA STI program provides access to the NASA Aeronautics and Space Database and its public interface, the NASA Technical Report Server, thus providing one of the largest collections of aeronautical and space science STI in the world. Results are published in both nonNASA channels and by NASA in the NASA STI Report Series, which includes the following report types:

- TECHNICAL PUBLICATION. Reports of completed research or a major significant phase of research that present the results of NASA programs and include extensive data or theoretical analysis. Includes compilations of significant scientific and technical data and information deemed to be of continuing reference value. NASA's counterpart of peerreviewed formal professional papers but has less stringent limitations on manuscript length and extent of graphic presentations.

- TECHNICAL MEMORANDUM. Scientific and technical findings that are preliminary or of specialized interest, e.g., quick release reports, working papers, and bibliographies that contain minimal annotation. Does not contain extensive analysis.

- CONTRACTOR REPORT. Scientific and technical findings by NASA-sponsored contractors and grantees.
- CONFERENCE PUBLICATION. Collected papers from scientific and technical conferences, symposia, seminars, or other meetings sponsored or cosponsored by NASA.

- SPECIAL PUBLICATION. Scientific, technical, or historical information from NASA programs, projects, and missions, often concerned with subjects having substantial public interest.

- TECHNICAL TRANSLATION. Englishlanguage translations of foreign scientific and technical material pertinent to NASA's mission.

Specialized services also include creating custom thesauri, building customized databases, and organizing and publishing research results.

For more information about the NASA STI program, see the following:

- Access the NASA STI program home page at $<$ http://www.sti.nasa.gov>

- E-mail your question via the Internet to $<$ help@sti.nasa.gov>

- Fax your question to the NASA STI Help Desk at 301-621-0134

- Phone the NASA STI Help Desk at 301-621-0390

- Write to: NASA STI Help Desk NASA Center for AeroSpace Information 7115 Standard Drive Hanover, MD 21076-1320 
NASA/TM-2009-216113

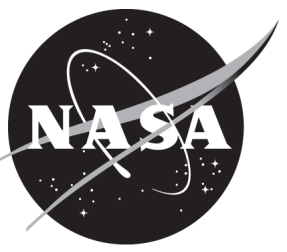

\section{Three-Dimensional Numerical Modeling of Magnetohydrodynamic Augmented Propulsion Experiment}

M.W. Turner and C.W. Hawk

The University of Alabama in Huntsville, Huntsville, Alabama

R.J. Litchford

Marshall Space Flight Center, Marshall Space Flight Center, Alabama

National Aeronautics and

Space Administration

Marshall Space Flight Center • MSFC, Alabama 35812 


\section{Acknowledgments}

This work was sponsored by the NASA Graduate Student Research Program under the direction of NASA Marshall Space Flight Center. The authors express the sincerest thanks to Robert Rhodes, Nobuomi Sakamoto, Nob Harada, John Lineberry, Jason Cassibry, and Jonathan Jones for their expert advice and assistance during the course of this work.

Available from:

NASA Center for AeroSpace Information

7115 Standard Drive

Hanover, MD 21076-1320

$301-621-0390$

This report is also available in electronic form at $<$ https://www2.sti.nasa.gov> 


\section{TABLE OF CONTENTS}

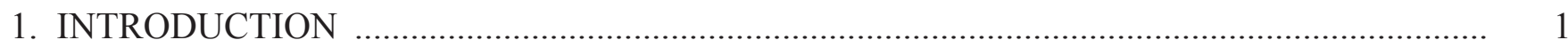

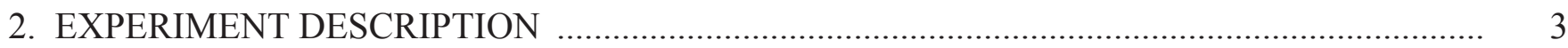

2.1 Hot Gas Source and Entrance Path ...................................................................................... 3

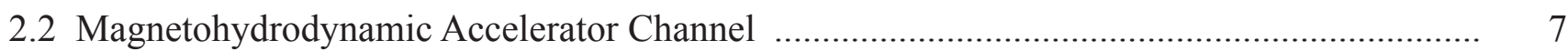

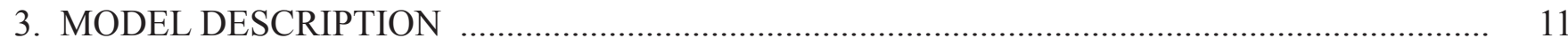

3.1 Baseline Modeling Capabilities .......................................................................................... 11

3.2 Extended Modeling Capabilities ....................................................................................... 18

3.3 Universal MGMHD Model Modifications and Improvements ............................................... 24

4. MAGNETOHYDRODYNAMIC AUGMENTED PROPULSION EXPERIMENT REGIME AND ANALYSIS PARAMETERS …………….................................................... 29

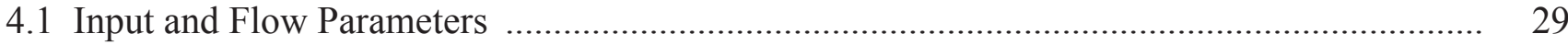

4.2 Thermodynamic/Electrophysical Regime ………………................................................. $\quad 30$

5. PARAMETRIC TRADE STUDY AND DESIGN OPTIMIZATION ANALYSES ....................... 32

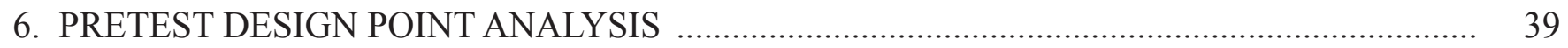

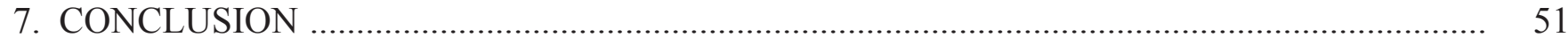

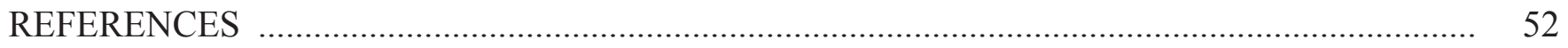





\section{LIST OF FIGURES}

1. Schematic of the NASA MSFC MAPX facility. The major flow path components are (1) $1.5-\mathrm{MW}_{\mathrm{e}}$ arc-heater, (2) seed injector and mixing chamber, (3) primary expansion nozzle, (4) 2-MW $\mathrm{e}$ MHD accelerator channel and 2-T electromagnet, (5) secondary nozzle, (6) windowed test section, and (7) nitrogen-driven ejector pump

2. Segmentation and assembly detail of the MSFC 1-MW $\mathrm{e}_{\mathrm{e}}$ multigas arc-heater

3. Exploded schematic of MAPX entrance flow path assembly ............................................. 5

4. Electric-to-thermal conversion efficiency with assumed nozzle cooling loses ...................... 6

5. Primary nozzle performance characteristics....................................................................... 6

6. Alternative design configuration for linear MHD accelerator channels: (a) Linear hall accelerator, (b) segmented Faraday accelerator, (c) series connected diagonal accelerator, and (d) diagonal conducting wall accelerator

7. Measured centerline magnetic field strength profiles at 2,400-A applied current

8. Schematic representation of MHD generator configurations in Cartesian coordinates:

(a) Diagonal conducting wall, (b) diagonal insulating sidewall, and (c) Faraday

9. Orientation of field vectors in a diagonally-connected MHD generator channel

10. Structural coding blocks for the UMM three-dimensional numerical model

11. Flowchart of omega.f routine for execution of the Gauss-Laguerre quadrature procedure

12. Structure of the thermoelectric property table created by the thermodynamic and electrical transport property submodels in the UMM code

13. Flowchart for the thermhd.f program-includes the thermodynamic calculations made by ceb.f and the electrical calculations made by econ.h

14. Illustration of UMM MHD block using the thermoelectrical data in three-dimensional calculations

15. Physical characteristics of the MAPX accelerator 


\section{LIST OF FIGURES (Continued)}

16. Major thermodynamic/thermoelectric parameters over regime of interest: (a) Temperature as a function of pressure and enthalpy, (b) electrical conductivity as a function of temperature and pressure, (c) electron number density as a function of temperature and pressure, and (d) electron mobility as a function of temperature and pressure

17. Axial variation of diagonalization angle, $\theta_{d}$, for axial current neutralized case with 100-A applied current. Definition of $\theta$ and $\theta_{d}$ illustrated above

18. Cross-sectional averaged (a) velocity and (b) temperature as a function of axial distance in the MAPX accelerator. Levels of applied current are indicated

19. Centerline total pressure as a function of axial position. Levels of applied current are indicated

20. Cross-sectional averaged transverse current density, $J_{y}$, as a function of axial position. Levels of applied current are indicated

21. Axial current density, $J_{x}$, as a function of axial position: (a) Cross-sectional averaged $J_{x}$ and (b) centerline $J_{x}$. Levels of applied current are indicated

22. Various power levels along the channel: (a) Accumulated total power, (b) push power, and (c) joule dissipated power

23. Cumulative global efficiency for the MAPX accelerator. Levels of applied current are indicated

24. Three-dimensional axial velocity, $u$, plotted at (a) 0.2, (b) 10, (c) 25, (d) 60, (e)80, and (f) $90.5 \mathrm{~cm}$ along the MAPX accelerator channel. The last position corresponds to the end of the powered region in the channel. The positive $J_{y}$ current density flows from the bottom electrode (background) to the top electrode (foreground) in a direction out of the page. All velocities are in $\mathrm{m} / \mathrm{s}$

25. Vector plots of cross-sectional secondary flow velocities for (a) $0.2 \mathrm{~cm}$, (b) $10 \mathrm{~cm}$, (c) $25 \mathrm{~cm}$, (d) $60 \mathrm{~cm}$, (e) $80 \mathrm{~cm}$, and (f) $90.5 \mathrm{~cm}$ with the axial velocity, u, shown as a background contour plot. Positive $J_{y}$ current flows from the bottom electrode to the top electrode. The length of the vectors is representative of their relative intensities. All velocities are in $\mathrm{m} / \mathrm{s}$

26. Three-dimensional temperature plots at various downstream locations in the MAPX accelerator channel: (a) $0.2 \mathrm{~cm}$, (b) $10 \mathrm{~cm}$, (c) $25 \mathrm{~cm}$, (d) $60 \mathrm{~cm}$, (e) $80 \mathrm{~cm}$, and (f) $90.5 \mathrm{~cm}$. Positive $J_{y}$ current density flows from the bottom electrode (background) to the top electrode (foreground) in accelerator mode 


\section{LIST OF FIGURES (Continued)}

27. Three-dimensional total pressure plots at various downstream locations: (a) $0.2 \mathrm{~cm}$, (b) $10 \mathrm{~cm}$, (c) $25 \mathrm{~cm}$, (d) $60 \mathrm{~cm}$, (e) $80 \mathrm{~cm}$, and (f) $90.5 \mathrm{~cm}$. Positive $J_{y}$ current flow is from the background to the foreground

28. Contour plots of the $J_{y}$ current density $\left(\mathrm{A} / \mathrm{cm}^{2}\right)$ at various downstream locations: (a) $0.2 \mathrm{~cm}$, (b) $10 \mathrm{~cm}$, (c) $25 \mathrm{~cm}$, (d) $60 \mathrm{~cm}$, (e) $80 \mathrm{~cm}$, and (f) $90.5 \mathrm{~cm}$. Positive $J_{y}$ current flow goes from the bottom electrode to the top electrode

29. Vector plots of cross-sectional current densities $J_{y}$ and $J_{z}$ at various downstream locations in the MAPX accelerator channel for the 100-A case: (a) $0.2 \mathrm{~cm}$, (b) $10 \mathrm{~cm}$, (c) $25 \mathrm{~cm}$, (d) $60 \mathrm{~cm}$, (e) $80 \mathrm{~cm}$, and (f) $90.5 \mathrm{~cm}$. Positive $J_{y}$ current flow is from the bottom to top electrodes

30. Vector plots of the cross-sectional current flow at various downstream locations for the 175-A case: (a) $45 \mathrm{~cm}$, (b) $60 \mathrm{~cm}$, (c) $65 \mathrm{~cm}$, (d) $70 \mathrm{~cm}$, (e) $80 \mathrm{~cm}$, and (f) $90.5 \mathrm{~cm}$. The positive $J_{y}$ current density is from the bottom to top electrode when in accelerator mode

31. Contour plot of channel temperature $(\mathrm{K})$ at various downstream locations for the 175-A case: (a) $25 \mathrm{~cm}$, (b) $45 \mathrm{~cm}$, (c) $60 \mathrm{~cm}$, (d) $70 \mathrm{~cm}$, (e) $80 \mathrm{~cm}$, and (f) $90.5 \mathrm{~cm}$. Positive $J_{y}$ current flow occurs from the bottom to top electrode when in accelerator mode

32. Three-dimensional axial current, $J_{x}$, plots at various downstream locations:

(a) $0.2 \mathrm{~cm}$, (b) $10 \mathrm{~cm}$, (c) $25 \mathrm{~cm}$, (d) $60 \mathrm{~cm}$, (e) $80 \mathrm{~cm}$, and (f) $90.5 \mathrm{~cm}$. The positive $J_{y}$ current flows from the bottom electrode (background) to top electrode (foreground) in accelerator mode 


\section{LIST OF TABLES}

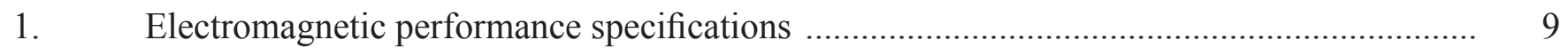

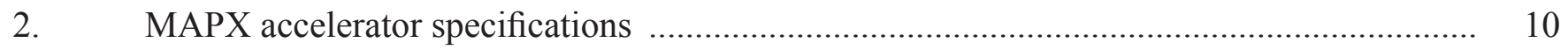

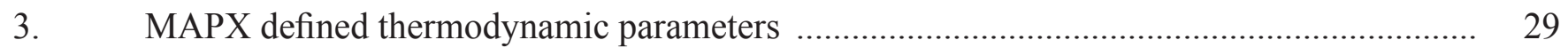

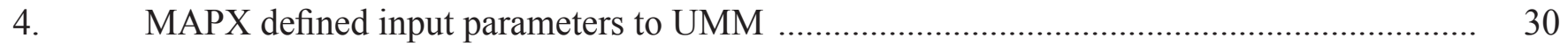




\section{LIST OF ACRONYMS AND SYMBOLS}

CEA Chemical Equilibrium Applications (computer code)

CFD computational fluid dynamics

DCW diagonal conducting wall

FAS full approximation storage

$\mathrm{I} / \mathrm{O} \quad$ input/output

MAPX Magnetohydrodynamic Augmented Propulsion Experiment

MGMHD multigrid magnetohydrodynamic (numerical model)

MHD magnetohydrodynamic

MSFC Marshall Space Flight Center

$\mathrm{NaK} \quad$ sodium potassium

PNS parabolized Navier-Stokes

TDMHD three-dimensional magnetohydrodynamic (computer code)

TM Technical Memorandum

UMM universal MGMHD model

UTSI University of Tennessee Space Institute 



\section{NOMENCLATURE}

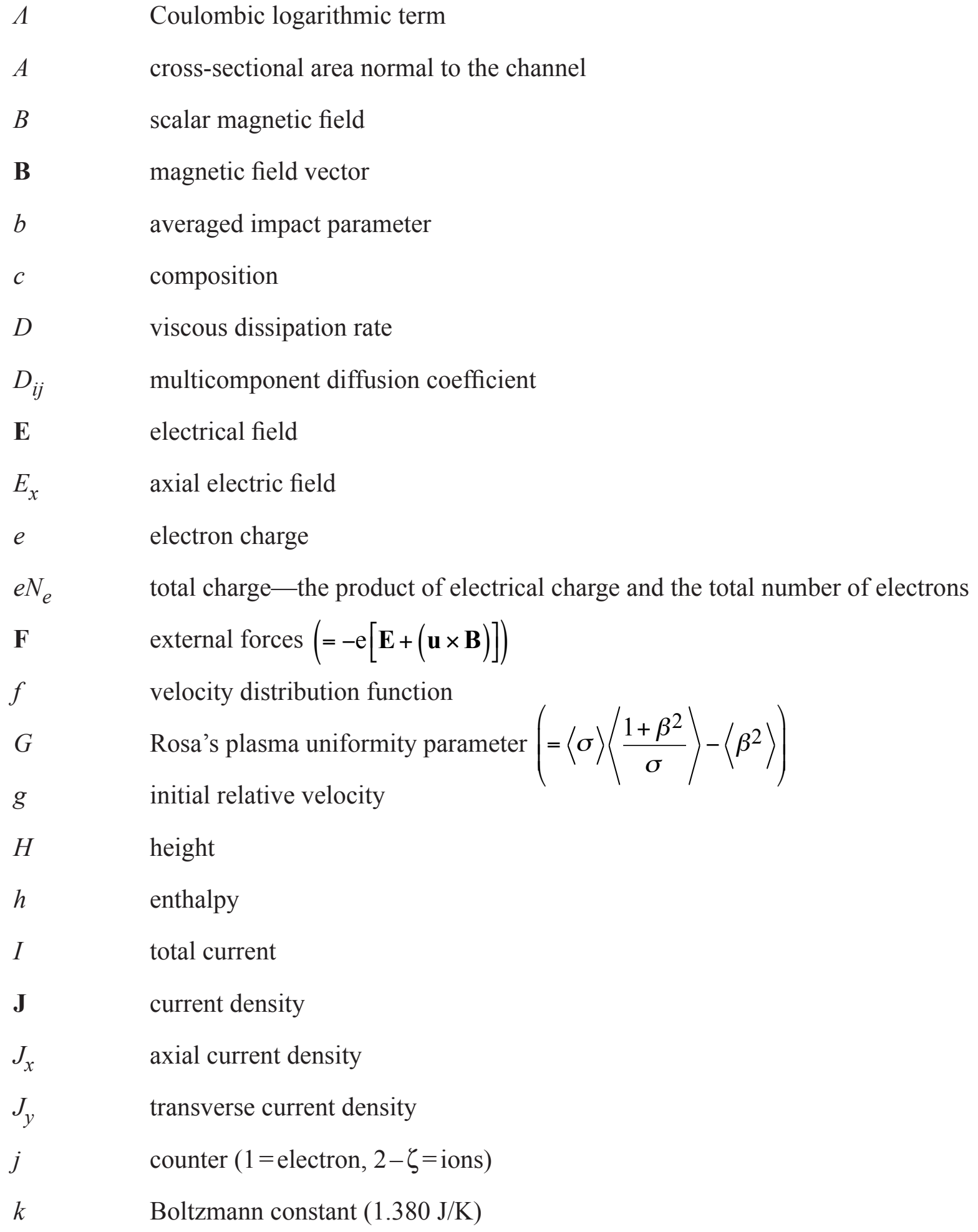




\section{NOMENCLATURE (Continued)}

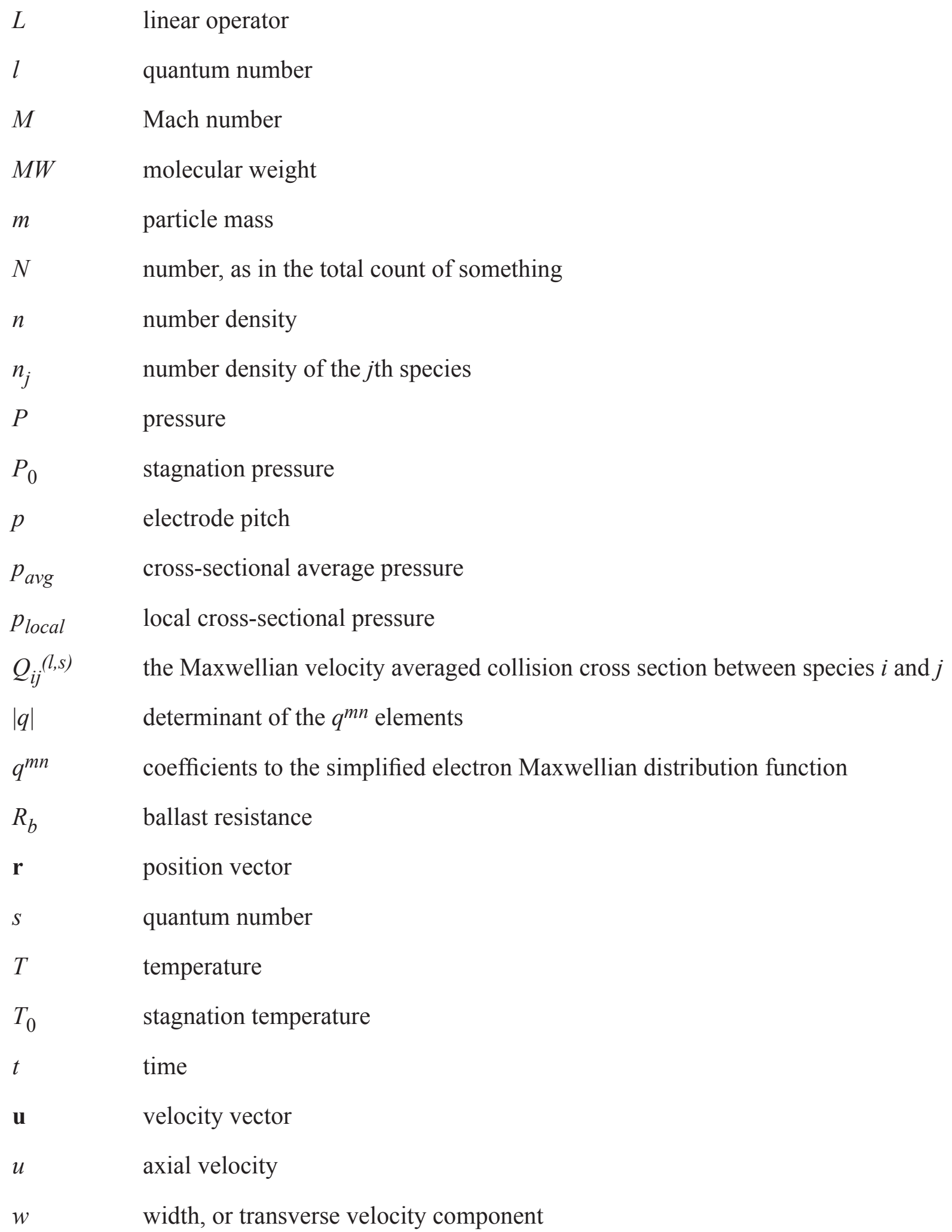




\section{NOMENCLATURE (Continued)}

$v$

Z

$Z_{j}$

$\beta$

$\gamma$

$\delta$

$\varepsilon$

$\eta$

$\theta$

$\theta_{d}$

$\theta_{w}$

$\kappa$

$\mu_{1}$

$\mu_{i j}$

$\rho$

$\sigma$

$\tau$

$\psi$

$\Omega_{i j}(l, s)$ transverse velocity component

atomic number

atomic number of species $j$

Hall parameter

ratio of specific heat

collisional energy parameter

dissipation rate, or small perturbation parameter

global electrical efficiency

electric field angle

diagonalization angle

wall angle

turbulence energy

laminar viscosity

reduced mass of the colliding species $i$ and $j$

mass density

electrical conductivity

shear stress

electric potential

collision integral of species $i$ and $j$ 

TECHNICAL MEMORANDUM

\section{THREE-DIMENSIONAL NUMERICAL MODELING OF MAGNETOHYDRODYNAMIC AUGMENTED PROPULSION EXPERIMENT}

\section{INTRODUCTION}

Magnetohydrodynamic (MHD) augmentation of thermal propulsion systems has been suggested as a plausible means of boosting exhaust velocity, and possibly improving overall specific energy attributes. In this way, one might hope to reduce fuel fraction and shrink vehicle size without sacrificing payload delivery capability. To obtain a meaningful improvement in fuel fraction, however, it can be shown that the electrical augmentation power must be greater in magnitude than the thermal power of the unaugmented source. Such considerations lead to some extremely daunting technical challenges, particularly in relation to the development of an onboard electrical power source with adequate power density characteristics. Nevertheless, several technological avenues can be identified that someday may lead to innovative, compact, high-power electrical energy sources possessing the required attributes, and exploratory pursuit of fundamental technical feasibility is not without credible justification. Moreover, MHD accelerator technology has potential dual use application in ground-based hypersonic wind tunnel facilities where the electrical power source weight is of little or no concern.

The essential requirement for using electromagnetic acceleration techniques is that the exhaust jet from the thermal propulsion source be electrically conductive. In practice, this can be accomplished by seeding the combustor flow of a chemical rocket with an alkali metal vapor, such as cesium, rubidium, potassium, and associated compounds. Because alkali metals have a relatively low ionization potential, the energy consumed in fully ionizing the seed is only a small fraction of the available thermal energy. Furthermore, the relatively low plasma working temperature is compatible with existing materials and regenerative cooling techniques. Using energetic rocket fuels, this method is known to produce supersonic plasma flows with an electrical conductivity on the order of $102 \mathrm{~S} / \mathrm{m}$, which is sufficient for evoking significant MHD interaction. At this level of MHD interaction, steady plasma acceleration is best invoked through externally imposed crossed electric and magnetic fields. This configuration gives rise to the so-called "crossed field MHD accelerator" in which the imposed Lorentz body force accelerates the flow.

Small prototypes for this class of plasma accelerator have been designed and built, but almost exclusively from the standpoint of producing a hypersonic wind tunnel rather than a propulsive device. Testing with these prototype devices has clearly demonstrated flow acceleration, but diagnostic limitations have prevented complete delineation of the fundamental physical phenomena. Many uncertainties remain including the relative importance of electromagnetic versus electrothermal effects, achievable accelerator efficiencies, achievable current densities, maximum sustainable axial electric field without interelectrode arcing, effect of near-wall velocity overshoot phenomena, effect of microarcing in the cold 
electrode boundary layer, multiterminal loading of a segmented Faraday channel versus twoterminal loading of a diagonal wall configuration, and thermal loading and erosive effects with respect to long-term channel survivability, to name a few.

Several years ago, NASA Marshall Space Flight Center (MSFC) initiated development of the Magnetohydrodynamic Augmented Propulsion Experiment (MAPX) for the purpose of resolving some of the critical technical issues associated with the use of MHD accelerators as thrust augmentation devices. A summary description of the project was previously published that included a thorough historical account of preceding MHD accelerator research programs, a detailed description of the experiment configuration, and results from a preliminary quasi-one-dimensional engineering performance analysis and design study. ${ }^{1}$ A recent status update for the project has summarized hardware design and development progress over the intervening time period. ${ }^{2}$ In parallel with this work, a three-dimensional numerical model based on the parabolized Navier-Stokes (PNS) approximation was developed to provide a comprehensive analysis of MHD accelerator performance. This new model was evolved from a preexisting three-dimensional numerical model previously validated for MHD generator performance, ${ }^{3}$ and was modified to incorporate the NASA Chemical Equilibrium with Applications (CEA) code ${ }^{4}$ as a means of calculating thermodynamic and species concentrations properties for the estimation of thermoelectric properties of partially ionized gases using a fundamental technique based on electron-neutral momentum transfer cross sections. ${ }^{5}$ The purpose of this Technical Memorandum (TM) is to summarize development of the parabolic three-dimensional numerical model, present pretest analyses and performance optimization results, and provide a recommended test configuration for initial MAPX tests. 


\section{EXPERIMENT DESCRIPTION}

The MAPX facility adapts a traditional linear MHD flow path configuration, as shown schematically in figure 1. First, the working fluid (i.e., nitrogen) is heated in a $1.5-\mathrm{MW}_{\mathrm{e}}$ segmented multigas archeater to a stagnation temperature, $T_{0} \approx 4,000-4,500 \mathrm{~K}$ at a stagnation pressure, $P_{0} \approx 10 \mathrm{~atm}$. The hot gas then enters a mixing chamber where alkali metal seed (i.e., NaK) is injected into the flow stream after which it is expanded through a primary nozzle to a Mach number in the range of $M \approx 1.25$ to 1.5. A 2- $\mathrm{MW}_{\mathrm{e}} \mathrm{MHD}$ accelerator directly increases the energy and momentum of the flow, which is further diffused in a secondary nozzle to obtain the maximum possible jet velocity. The secondary nozzle exhausts into a large windowed test section equipped with a stinger mounted stagnation probe or aero model. The test section is attached to a nitrogen-driven ejector pump designed to maintain a backpressure in the range of 1 to 3 psia.

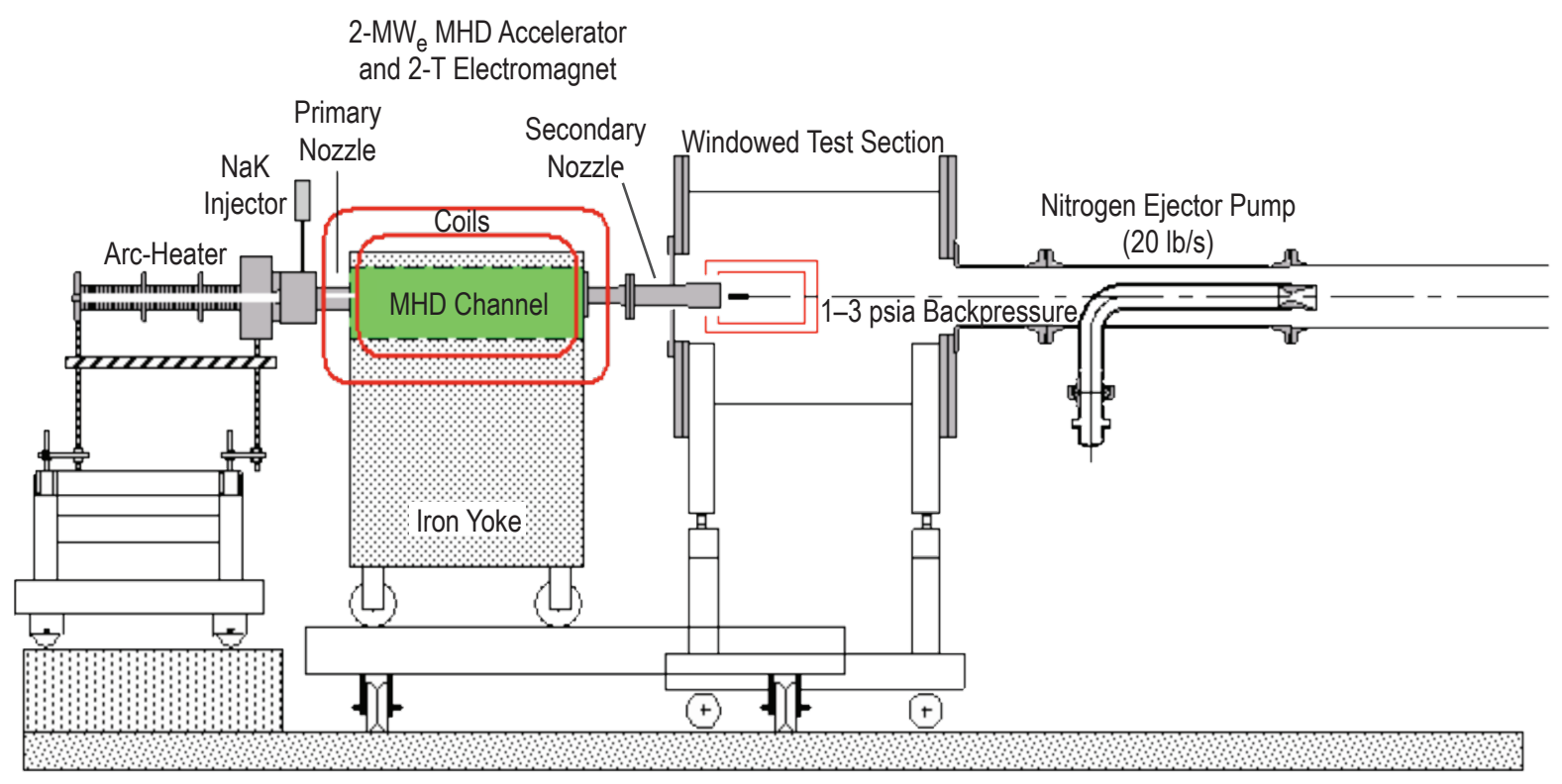

Figure 1. Schematic of the NASA MSFC MAPX facility. The major flow path components are (1) 1.5- $\mathrm{MW}_{\mathrm{e}}$ arc-heater, (2) seed injector and mixing chamber, (3) primary expansion nozzle, (4) 2-MW $\mathrm{MHD}$ accelerator channel and 2-T electromagnet, (5) secondary nozzle, (6) windowed test section, and (7) nitrogen-driven ejector pump.

\subsection{Hot Gas Source and Entrance Path}

The hyperthermal stagnation conditions are generated by a $1.5-\mathrm{MW}_{\mathrm{e}}$ (nominal) segmented multigas arc-heater, which operates in a wall-stabilized, constricted arc, dc discharge mode. The arc-heater is energized by a saturable reactor dc power supply that can sustain a continuous operating power of 
$0.75 \mathrm{MW}_{\mathrm{e}}$ on an indefinite basis and can deliver an intermittent power burst of $1.5 \mathrm{MW}_{\mathrm{e}}$ for 5 to $10 \mathrm{~min}$. The purpose of the thermal driver is to maximize the MHD interaction by delivering the highest possible mass throughput and maximizing the accelerator channel size.

This particular arc-heater has a 1-in internal bore diameter and follows the traditional segmentation design philosophy, whereby alternating conductor/insulator wafers are stacked together to form the full length assembly, as illustrated in figure 2. The 3/8-in-thick, heat-conducting copper segments are water cooled and are separated by boron nitride insulators in stacked pack subassemblies, which are held securely together by four INCONEL ${ }^{\circledR}$ tie rods. These subassembly packs are then attached in a sequential manner to form the full arc-heater column, which spans an overall length of $\approx 1 \mathrm{~m}$ in the three-pack configuration shown. The working gas is injected tangentially through four 0.048 -in jets in a primary gas injection segment near the rear of the arc-heater, and a dc arc discharge is established between a tungsten cathode button in the rear sealing flange and a copper anode ring at the arc-heater exhaust. A magnetic spin coil is located around the anode ring to induce continuous rotation of the arc attachment point.
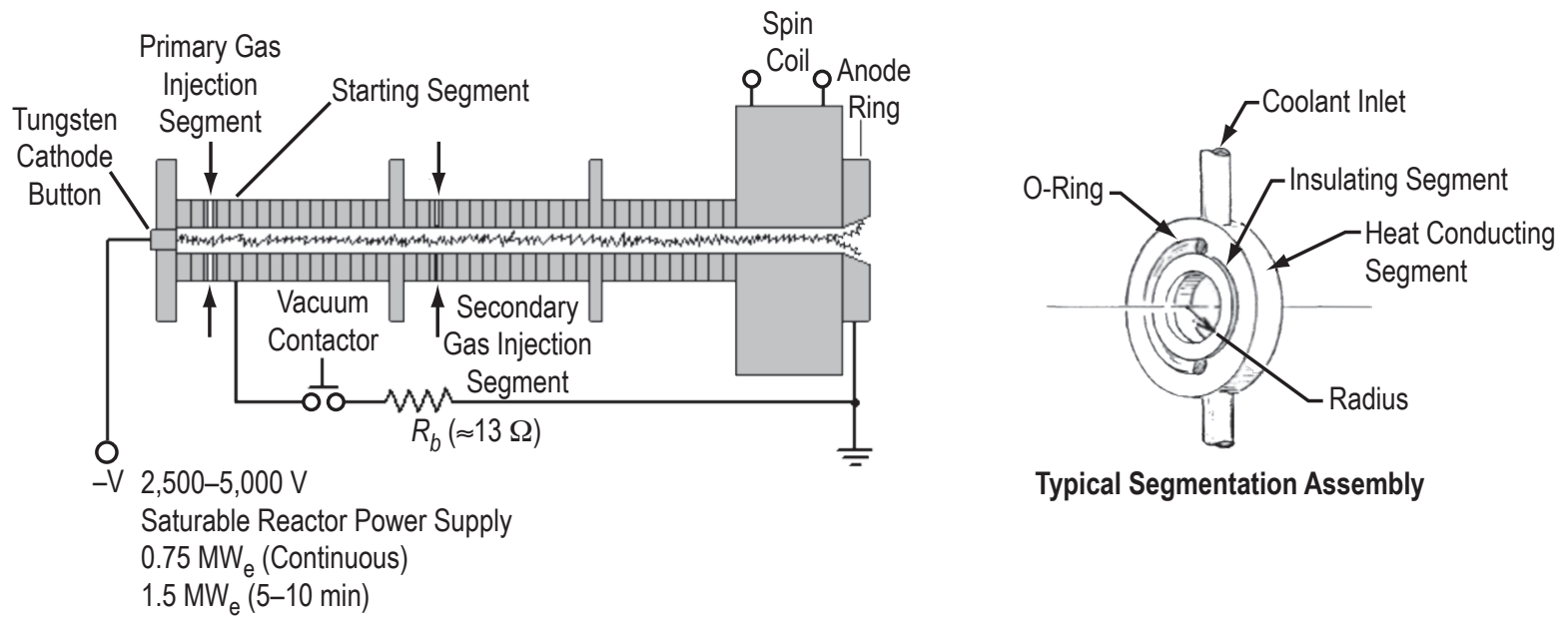

Figure 2. Segmentation and assembly detail of the MSFC 1-MW $\mathrm{e}_{\mathrm{e}}$ multigas arc-heater.

An exploded schematic of the entrance flow path assembly is shown in figure 3. This assembly accomplishes the following primary functions: (1) Electrical isolation of the arc-heater from the MHD accelerator and circular-to-square flow path transition, (2) NaK seed injection and mixing, and (3) flow acceleration via the nozzle. The isolation flange is designed for heat sink operation and is an expendable item, with the other components being water-cooled copper pieces intended for extended service. An alumina insert is located inside the isolation flange (as shown in fig. 3) that provides the geometric transition of the flow passage from the circular geometry of the arc-heater exit to the rectangular geometry of the MHD accelerator. This geometric transition is facilitated by the internal loft (flow area distribution) of the alumina insert that gradually transforms from circular to square. It is also worth noting that, because the circular-to-square transition occurs along the length of the isolation flange assembly without a change in diameter (width), the cross-sectional area increases by a factor of $4 / \pi$. 


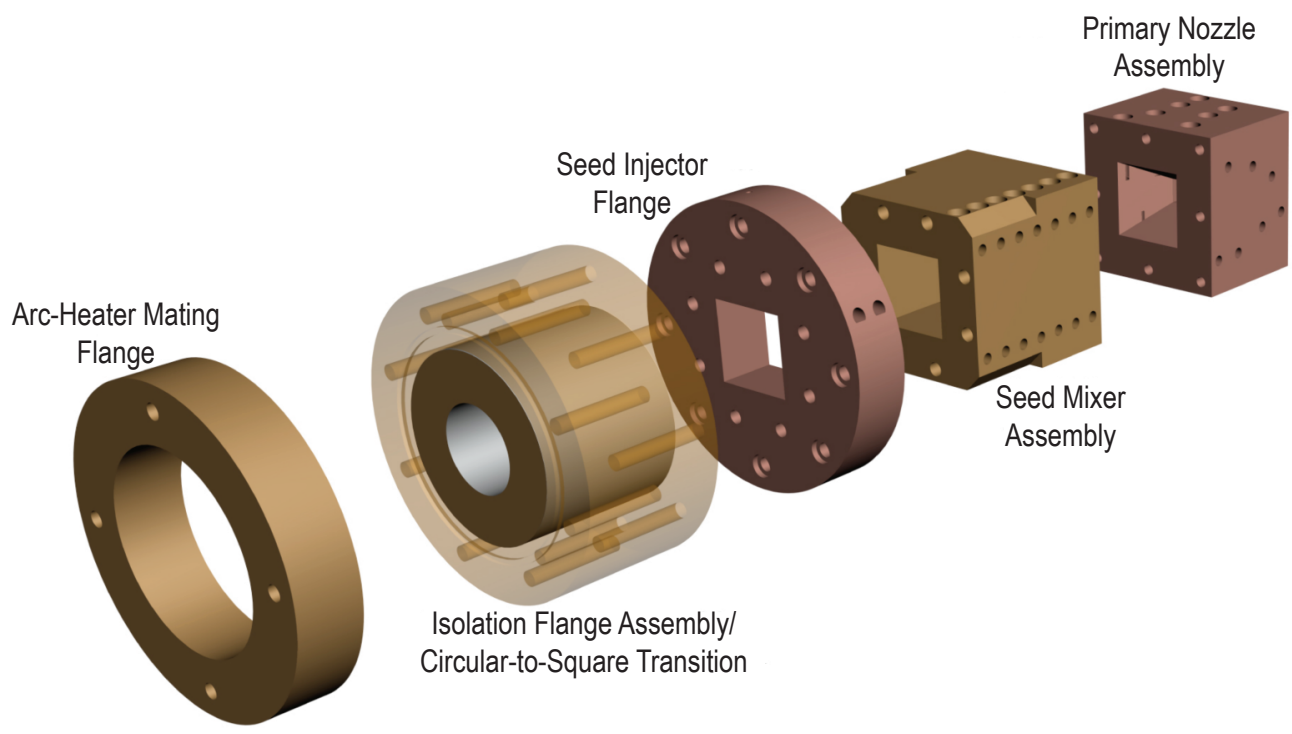

Figure 3. Exploded schematic of MAPX entrance flow path assembly.

Over the years, a considerable amount of historical performance data have been accumulated and cataloged for this particular arc-heater, and the resulting database can be used to project performance characteristics at representative MAPX test conditions. Projected variation in electric-to-thermal conversion efficiency with applied electrical power, for instance, is shown in figure 4, along with some recently acquired experimental data. These experimental efficiencies were inferred from calorimeter measurements obtained during high flow rate nitrogen runs using an uncooled graphite nozzle with a 5/8-in $\varnothing$ throat. In general, the projected efficiencies were within one to two percentage points of the experimentally observed values over the examined power range.

Preliminary analysis, assuming an applied electrical power of $1.1 \mathrm{MW}_{\mathrm{e}}$ and nozzle losses of no more than $20 \%$ of the available total enthalpy, indicated that satisfactory conditions could be achieved with $130 \mathrm{~g} / \mathrm{s}$ of nitrogen and $1.5 \% \mathrm{NaK}$ (by weight) using a $0.567 \times 0.567 \mathrm{in}^{2}$ throat with an area expansion ratio of $A / A^{*}=1.142297$. The $20 \%$ nozzle heat loss limit was derived from practical experience with water-cooled copper nozzles in high-temperature combustors. The primary nozzle performance characteristics were estimated using a modified version of the CEA code, which incorporates a method for computing plasma electrical transport properties. At the 1.1-MW $\mathrm{MW}_{\mathrm{e}}$ operating design point, the archeater electric-to-thermal conversion efficiency was experimentally determined to be $\approx 63 \%$, yielding an equilibrium total temperature of $3,302 \mathrm{~K}$ at the primary nozzle entrance. Anticipated thermodynamic/ electrical conditions at the accelerator entrance are summarized in figure 5 as a function of actual primary nozzle performance. These chemical equilibrium calculations indicate that acceptable accelerator inlet conditions can be attained even for the worst-case performance scenario. In this performancelimiting situation, the inlet static temperature and electrical conductivity are $2,397 \mathrm{~K}$ and $11 \mathrm{~S} / \mathrm{m}$, respectively. 


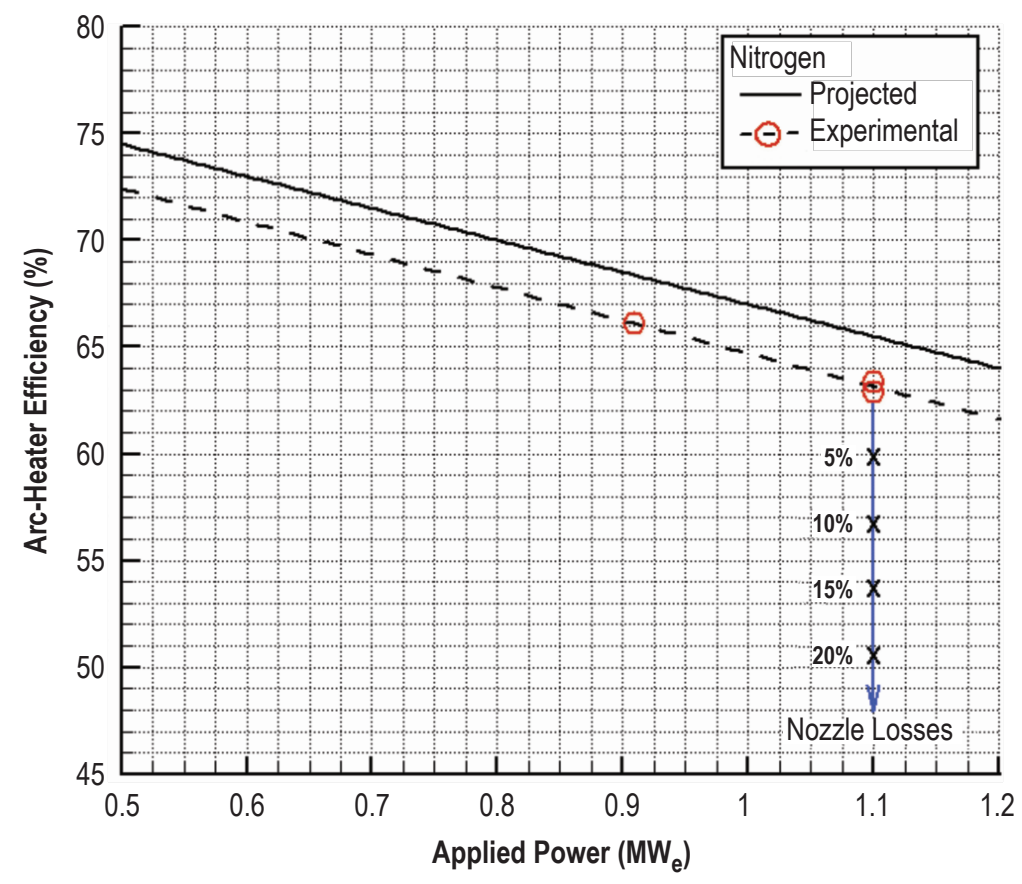

Figure 4. Electric-to-thermal conversion efficiency with assumed nozzle cooling loses.

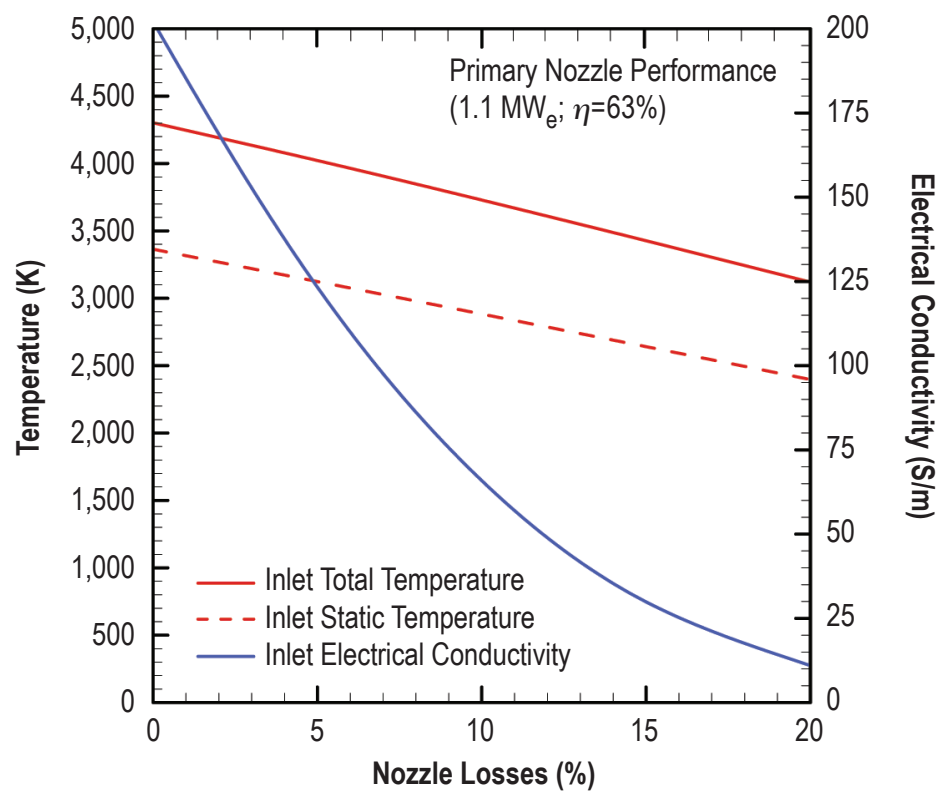

Figure 5. Primary nozzle performance characteristics. 


\subsection{Magnetohydrodynamic Accelerator Channel}

\subsubsection{Electrical Loading Configuration}

Alternative configurations for linear MHD accelerator channels are depicted in figure 6 where the optimal MHD accelerator configuration is determined by the ultimate application needs. From a performance standpoint, the Hall configuration (fig. 6(a)) is more effective for low-density flows whereas the Faraday configuration (fig. 6(b)), with segmentation to neutralize the Hall current, is superior for highdensity flows. The major drawback of the Faraday configuration, however, is the separate power conditioning required for each electrode pair which leads to a complex and expensive system. In many cases, particularly flight applications, multiterminal loading is not practical.

Alternative two-terminal loading schemes have been proposed to avoid the multiterminal complications while attempting to reap the major benefit associated with the Faraday configuration; i.e., Hall current neutralization. For example, the standard segmented Faraday channel may be externally diagonalized in a series connected scheme (fig. 6(c)), or one could adopt a diagonal conducting wall (DCW) configuration in which slanted window frame-like electrode elements are stacked with thin insulators to form a complete channel (fig. 6(d)). The DCW configuration not only simplifies fabrication and improves strength but provides superior performance to the externally shorted (i.e., series connected) device by allowing current to flow to the sidewalls.

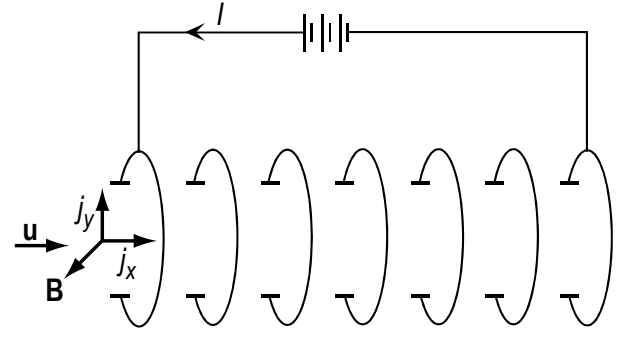

(a) Linear Hall Accelerator

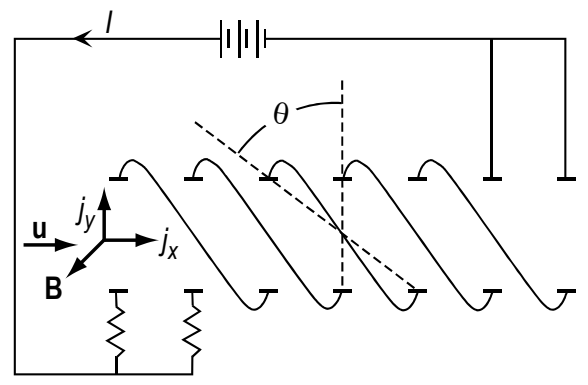

(c) Series Connected Diagonal Accelerator

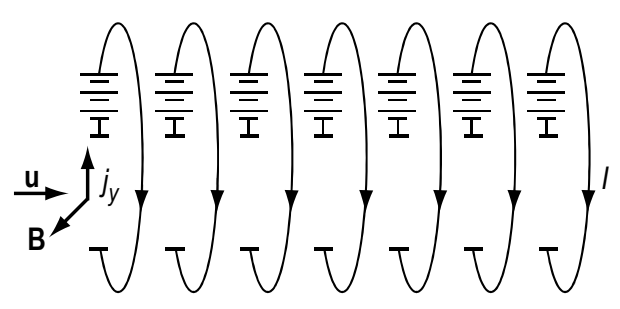

(b) Segmented Faraday Accelerator

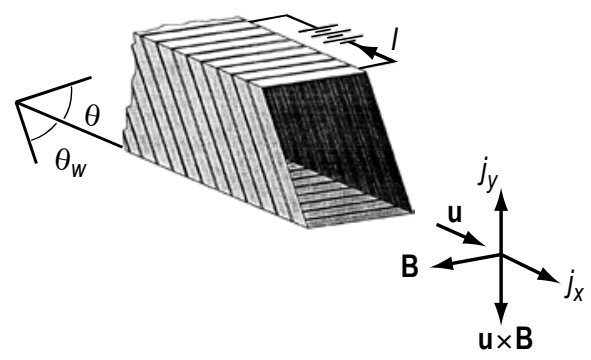

(d) Diagonal Conducting Wall Accelerator

Figure 6. Alternative design configuration for linear MHD accelerator channels: (a) Linear hall accelerator, (b) segmented Faraday accelerator, (c) series connected diagonal accelerator, and (d) diagonal conducting wall accelerator. 
Although the DCW configuration is the overall best candidate for flight implementation, the MAPX design was based on an externally diagonalized series connected configuration for reasons of cost and flexibility (e.g., effective wall angle adjustability).

\subsubsection{Engineering Design and Performance Analysis}

Numerous investigations have clearly established that MHD channel flows are subject to significant three-dimensional effects. Thus, averaging the governing MHD equations (magnetic Reynolds number $<<1$ ) to obtain a quasi-one-dimensional engineering model requires the adoption of major simplifying assumptions. Nevertheless, many of these assumptions including wall friction, wall heat flux, and near-electrode voltage drops may be approximately accounted for through the introduction of appropriate physical wall functions for the boundary layer. 6,7

Engineering design and performance analyses of the MAPX accelerator were carried out using a legacy code based on one such approach. This engineering code was initially developed within the Energy Conversion Division at the University of Tennessee Space Institute (UTSI) in support of the Department of Energy MHD Commercial Power program. Over the years, the code was evolved and expanded to encompass a range of generator and accelerator loading configurations. In most respects, the development is similar to that described for other nonperfect-gas, quasi-one-dimensional analyses, the principle idiosyncrasies being associated with the physical submodeling. The code solves the governing internal duct flow equations for conservation of mass, momentum, and energy together with the equation of state and boundary layer wall functions using a fourth-order Runge-Kutta numerical integration scheme. It uses a real-gas equation of state and assumes local thermodynamic equilibrium as predicted by the CEA code with appropriate modifications for computing electrical transport properties. Values for the empirical constants associated with various physical submodels have been established through extensive benchmarking experience.

Heat transfer and frictional wall losses are computed intrinsically in the code and require input of the wall temperature and roughness height. Near-wall electrical losses are also treated intrinsically through integration of the conductivity profile as defined by velocity and thermal boundary layer correlations for fully turbulent flow. This correlation computes the boundary layer growth along the MHD accelerator duct through definition/input of the initial boundary layer height and shear (viscosity as a function of temperature). The velocity and temperature profiles are taken as $1 / n$ power-law distributions. This approach also relies on user specification of the Rosa $G$ factor to account for plasma nonuniformities and effective voltage drop. A value of $G \approx 2$ was anticipated based on past experience and was therefore utilized for engineering design and performance analyses purposes.

Actual accelerator performance depends, of course, on constraints imposed by the available magnet and power supply equipment. To meet the research goals of this program, a water-cooled, 2-T electromagnet was acquired from UTSI and refurbished to support general MHD research at MSFC. The specifications for this magnet are summarized in table 1. A new 3,000-A, 75-V dc power supply was acquired to power the magnet, and the entire system has been installed and integrated into the MAPX flow train. A shakedown test of the electromagnet system was conducted and the measured field profile at 2,400 $\mathrm{A}$ is shown in figure 7. A 2-MW $\mathrm{MW}_{\mathrm{e}}$, high-voltage dc power supply was also acquired and installed to power the accelerator. The voltage on the unit is variable to $10 \mathrm{kV}$ and is capable of delivering $300 \mathrm{~A}$ at $6,700 \mathrm{~V}$. 
Table 1. Electromagnetic performance specifications.

\begin{tabular}{lc}
\hline Magnetic field strength & $2 \mathrm{~T}$ \\
Air gap & $4 \mathrm{in}$ \\
Pole cap length & $36 \mathrm{in}$ \\
Voltage & $65 \mathrm{~V}$ \\
Maximum current & $2,400 \mathrm{~A}$ \\
Cooling water (70 psig supply) & $50 \mathrm{gal} / \mathrm{min}$ \\
\hline
\end{tabular}

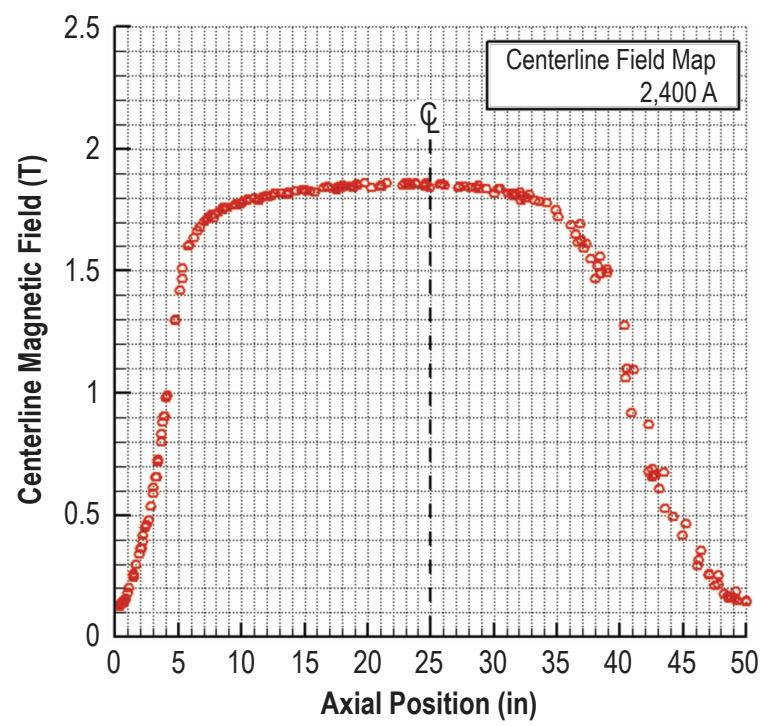

Figure 7. Measured centerline magnetic field strength profiles at 2,400-A applied current.

The detailed design process entailed several iterative calculations in an attempt to optimize stagnation pressure rise by varying load current, channel divergence, and channel length. These engineering calculations were carried out utilizing the measured centerline magnetic field profile at 2,400 A. The height-to-width aspect ratio was unity at the inlet, and the E-field and B-field walls were diverged to accommodate boundary layer growth and flow expansion. Preliminary analysis of the axial current neutralized operation indicated that the optimal diagonalization angle was near $\theta \approx-45^{\circ}$. The resulting physical specifications selected for the accelerator design based on this extensive engineering study are summarized in table 2 . 
Table 2. MAPX accelerator specifications.

\begin{tabular}{lc}
\hline Inlet height $\times$ width & $1.6 \times 1.6 \mathrm{~cm}^{2}$ \\
Channel divergence & $1.0^{\circ}$ \\
Electrode width & $1.0 \mathrm{~cm}$ \\
Insulator width & $0.5 \mathrm{~cm}$ \\
Active length & $90 \mathrm{~cm}$ \\
Powered electrodes & 60 \\
Total length & $96 \mathrm{~cm}$ \\
Total electrodes & 65 \\
Exit height $\times$ width & $3.6 \times 3.6 \mathrm{~cm} 2$ \\
Seed (NaK) & $1.5 \%$ \\
Nitrogen flow rate & $130 \mathrm{~g} / \mathrm{s}$ \\
$T_{0, \text { in }}$ & $3,120 \mathrm{~K}$ \\
$P_{0, \text { in }}$ & $8.5 \mathrm{~atm}$ \\
$u_{\text {in }}$ & $1,312 \mathrm{~m} / \mathrm{s}$ \\
$\sigma_{\text {in }}$ & $25 \mathrm{~S} / \mathrm{m}$ \\
$\beta_{\text {in }}$ & 0.7 \\
\hline
\end{tabular}




\section{MODEL DESCRIPTION}

It is widely recognized that the current and electric field structure in MHD devices is inherently three-dimensional and spatial separation of physical processes is not warranted in general. Generally, it is sufficient to invoke an infinite segmentation assumption (i.e., the streamwise variation in electrical properties is small in comparison to transverse variations) and couple an approximate cross-plane electrical model with a three-dimensional flow analysis, as exemplified for generator configurations by Bityurin et al. ${ }^{8}$ and Ahluwalia et al. ${ }^{9}$

Here, we adopt the multigrid magnetohydrodynamic (MGMHD) model ${ }^{3}$ previously developed by Argonne National Laboratory for the analysis of MHD generators and adapt it for the MHD accelerator configurations. The resulting development, to be described in this TM, is designated as the universal MGMHD model (UMM). ${ }^{10}$ UMM is a PNS computational fluid dynamics- (CFD-) based methodology capable of simulating both generator and accelerator modes of operation in Hall, Faraday, and diagonal configurations. The numerical model utilizes the CEA code for calculating thermodynamic properties and species concentrations and adopts a fundamental kinetics model for the estimation of electrical transport properties from experimental electron-neutral momentum transfer cross sections. Section 3 provides a brief overview of the MGMHD numerical model, including a detailed discussion of its capabilities and limitations.

\subsection{Baseline Modeling Capabilities}

The MGMHD computer code is an updated version of the single-grid, three-dimensional magnetohydrodynamic (TDMHD) code developed at the Argonne National Laboratory in 1982 for the analysis of MHD generators and diffusers. ${ }^{3}$ The MGMHD code retains the TDMHD formulation of threedimensional partial differential equations for flow and electrical fields but incorporates an advanced multigrid solution algorithm to reduce computer convergence time. The MHD equation set comprises the mass continuity equation, three momentum equations, the energy equation, two turbulence model equations, and Maxwell's equations. Turbulence is represented by a two-equation model $(\kappa-\varepsilon)$ in which partial differential equations are solved for the turbulence energy $(\kappa)$ and its dissipation rate $(\varepsilon)$. Lastly, the MGMHD code provides two unique features: (1) A full approximation storage (FAS), block implicit multigrid, finite-difference solution procedure for the cross-stream hydrodynamic equations and (2) a FAS multigrid, finite-difference solution procedure for cross-stream electrical potential equations.

\subsubsection{Fluid Dynamic Model}

Flow processes in an MHD generator can be generally represented by the three-dimensional Navier-Stokes equations. In an MHD channel, however, the flow is predominantly in the stream-wise direction, which allows for certain simplifications when considering the order of magnitude of various terms. The simplification made here is referred to as the PNS approximation, which is akin to the boundary layer approximation made in solving problems such as the flat-plate flow, and consists of the 
following major assumptions: (1) Diffusion flux in the stream-wise coordinate may be neglected and (2) the pressure gradient in the axial momentum equation is assumed to be uniform over the channel cross section.

The PNS approximation permits the solution to be marched forward plane-by-plane from the inlet to the exit of the channel. This approximation does not introduce any significant errors when the flow is predominantly in one direction, and it eliminates the need to iterate between the inlet and exit of the channel, thereby making the calculation converge more quickly. In this case, the pressure field, $P$, is split into two components: (1) $p_{\text {local }}$, the local cross-sectional pressure, and (2) $p_{\text {avg, }}$, the cross-sectional average pressure, which, as the following equation shows, is only a function of the axial coordinate:

$$
P(x, y, z)=p_{\text {local }}(x, y, z)+p_{\text {avg }}(x)
$$

The coordinate system utilized by the MGMHD numerical model is Cartesian; wherein, the mass continuity, momentum, and energy (enthalpy) equations are represented by the following equation set:

Mass:

$$
\frac{\partial}{\partial x}(\rho u)+\frac{\partial}{\partial y}(\rho u)+\frac{\partial}{\partial z}(\rho u)=0
$$

$x$-Momentum (axial direction in channel):

$$
\frac{\partial}{\partial x}(\rho u u)+\frac{\partial}{\partial y}(\rho v u)+\frac{\partial}{\partial z}(\rho w u)=-\frac{\partial p_{a v g}}{\partial x}+\frac{\partial \tau_{x y}}{\partial y}+\frac{\partial \tau_{x z}}{\partial z}+J_{y} B
$$

-Momentum:

$$
\frac{\partial}{\partial x}(\rho u v)+\frac{\partial}{\partial y}(\rho v v)+\frac{\partial}{\partial z}(\rho w v)=-\frac{\partial p_{l o c a l}}{\partial y}+\frac{\partial \tau_{y y}}{\partial y}+\frac{\partial \tau_{y z}}{\partial z}-J_{y} B
$$

$z$-Momentum:

$$
\frac{\partial}{\partial x}(\rho u w)+\frac{\partial}{\partial y}(\rho v w)+\frac{\partial}{\partial z}(\rho w w)=-\frac{\partial p_{l o c a l}}{\partial z}+\frac{\partial \tau_{z y}}{\partial y}+\frac{\partial \tau_{z z}}{\partial z}
$$

Enthalpy (energy):

$$
\frac{\partial}{\partial x}(\rho u h)+\frac{\partial}{\partial y}(\rho v h)+\frac{\partial}{\partial z}(\rho w h)=-\frac{\partial q_{y}}{\partial y}-\frac{\partial q_{z}}{\partial z}+u \frac{\partial p_{\text {avg }}}{\partial x}+v \frac{\partial p_{\text {local }}}{\partial y}+w \frac{\partial p_{\text {local }}}{\partial z}+\frac{J^{2}}{\sigma}+D
$$

where the viscous dissipation rate, $D$, is calculated as follows:

$$
D=\left(\mu_{l}+\mu_{t}\right)\left[2\left(\left(\frac{\partial w}{\partial z}\right)^{2}+\left(\frac{\partial v}{\partial y}\right)^{2}\right)+\left(\frac{\partial w}{\partial y}+\frac{\partial v}{\partial z}\right)^{2}+\left(\frac{\partial u}{\partial z}\right)^{2}+\left(\frac{\partial u}{\partial y}\right)^{2}\right]
$$


In the preceding equations, $u, v$, and $w$ are the $x, y$, and $z$ components of the $\mathbf{u}$ velocity vector, respectively. The symbol $\rho$ represents mass density, $\tau$ represents shear stress, $\sigma$ is electrical conductivity, $h$ is enthalpy, $J$ is current density, and $B$ is the magnetic field (here, $B$ represents only the scalar magnitude of the magnetic field). The MGMHD code assumes steady state; therefore, any time-dependent fluctuations will not be captured with this numerical model. Furthermore, note that equation (6) uses enthalpy instead of $c_{p} T$, the product of specific heat and temperature, in the energy equation. This is because the MGMHD code advances the energy equation using enthalpy, not temperature. Advancing the energy equation using temperature requires that one assume a constant specific heat (i.e., taking $c_{p} T$ out of the derivative in energy equation), which is not true in some temperature ranges. Advancing the energy equation using enthalpy (or internal energy) requires no such assumption.

\subsubsection{Electrical Model}

Slow calculation of the electrical potential solution is one difficulty in performing a complete three-dimensional MHD analyses with finite channel segmentation. In this section, the cross-sectional infinite segmentation electrical model, developed by Ahluwalia ${ }^{9}$ and utilized in the MGMHD model, is summarized. In the infinite segmentation model, axial variations of the flow and electrical variables are negligible in comparison to their cross-plane variations. Thus, a solution may be obtained by marching stepwise from the entrance of the channel to the exit. This model was successfully applied to the threedimensional analysis of Faraday, diagonal insulating, and diagonal conducting sidewall MHD generators and carefully validated against experimental data with good success. The electrical model is coupled to the fluid dynamic model presented above. More extensive discussion and detailed derivations may be found elsewhere. ${ }^{9,10}$

In the MGMHD code, the electrical governing equations consist of Maxwell's steady-state equations and Ohm's Law. The electrical field, E, and the current density, $\mathbf{J}$, are obtained by solving the following equations:

$$
\begin{aligned}
& \nabla \times \mathbf{E}=0, \\
& \nabla \cdot \mathbf{J}=0,
\end{aligned}
$$

and

$$
\mathbf{J}=\sigma(\mathbf{E}+\mathbf{u} \times \mathbf{B})-\frac{\beta}{B} \mathbf{J} \times \mathbf{B} .
$$

In the above equation, $\beta$ is the Hall parameter. As typical for MHD channel flows, these equations are valid for low magnetic Reynolds number flows, where the induced magnetic can be neglected. Equations (8) and (9) make it possible to define an electric potential, $\psi$, such as

$$
\mathbf{E}=-\nabla \psi,
$$

which, because of the steady-state assumption, effectively reduces the vector problem to that of a scalar. Also, note that the MGMHD code assumes that the magnetic field, $\mathbf{B}$, is sectionally uniform and oriented in the positive $z$-direction, as shown in figures 8 and 9 . 

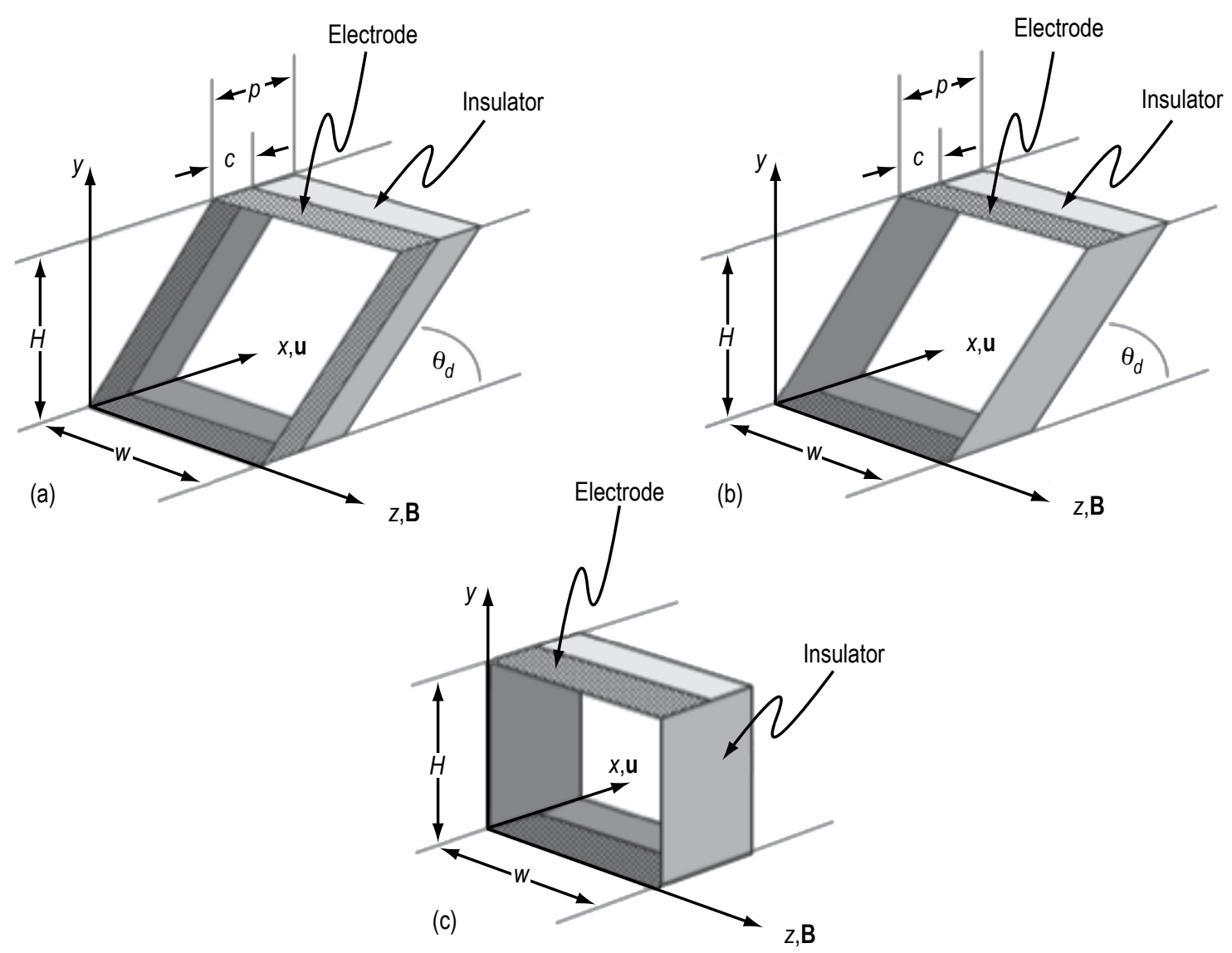

Figure 8. Schematic representation of MHD generator configurations in Cartesian coordinates: (a) Diagonal conducting wall, (b) diagonal insulating sidewall, and (c) Faraday.

The infinite segmentation model is constructed from equations (8) and (9) by neglecting axial variations of the flow and electrical variables in comparison with their cross-plane variations. Consequently, the axial electric field, $E_{x}$, is assumed to be constant in a cross-sectional plane. By defining a set of oblique coordinates in the general case of a diagonal connection, the electric potential can therefore be represented by the relation

$$
\psi=-\left(x-y \cot \theta_{d}\right) E_{x}+\varphi(y, z),
$$

where $\theta_{d}$ is the diagonalization angle (measured in a counterclockwise direction from the positive $x$-axis), and $\varphi(y, z)$ is a two-dimensional potential. The first term on the right side of equation (12) represents the contribution of the axial ( $x$-axis) electric field. Substituting equation (12) into equations (8)(10) results in the following equation for the two-dimensional function, $\varphi(y, z)$ :

$$
\frac{\partial}{\partial y}\left(\sigma_{n} \frac{\partial \varphi}{\partial y}\right)+\frac{\partial}{\partial z}\left(\sigma_{n} \frac{\partial \varphi}{\partial z}\right)=E_{x} \frac{\partial}{\partial y}\left[\left(\beta-\cot \theta_{d}\right) \sigma_{n}\right]-\frac{\partial}{\partial y}\left[\sigma_{n} B(u-\beta v)\right],
$$




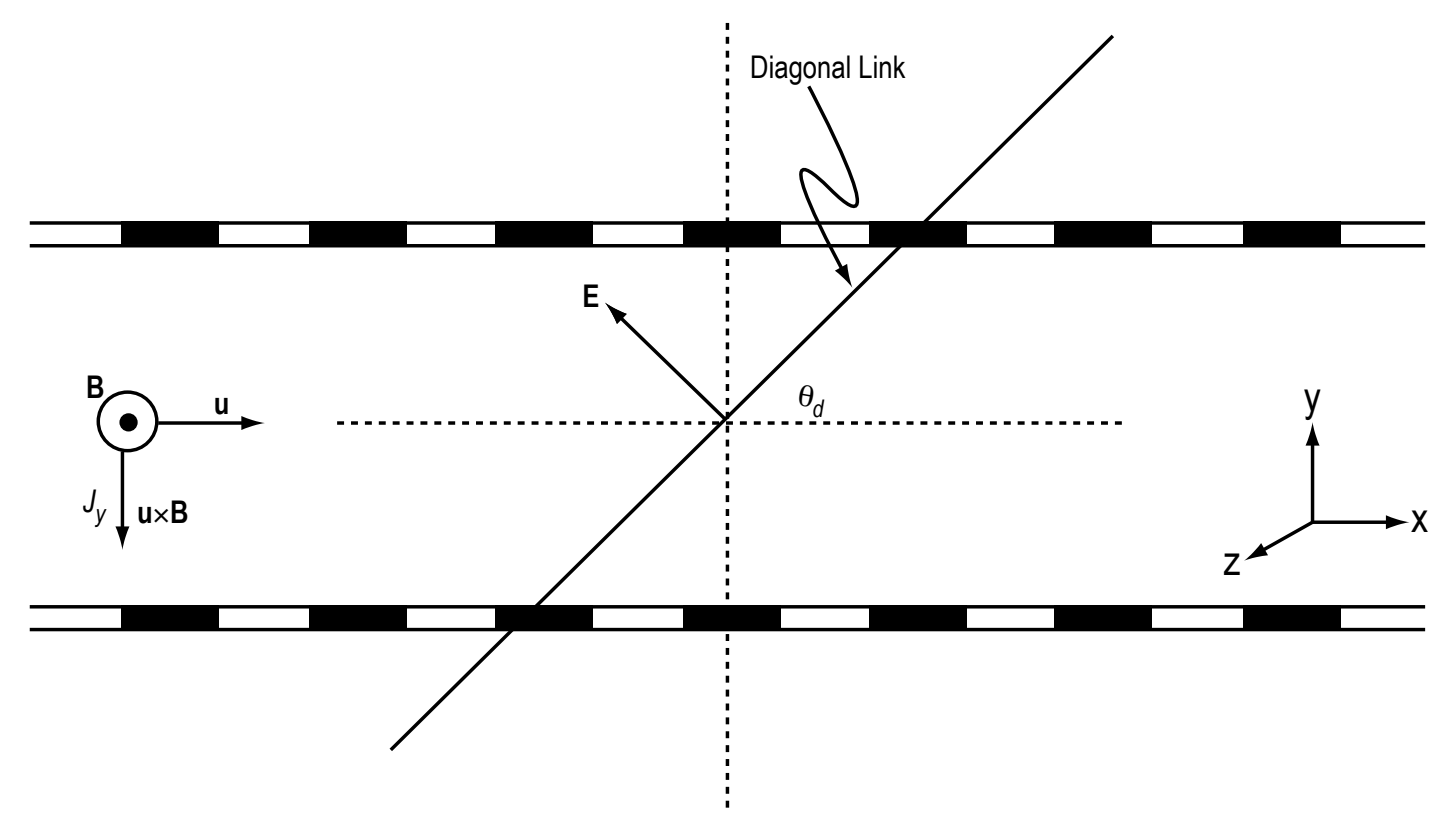

Figure 9. Orientation of field vectors in a diagonally-connected MHD generator channel.

where $\sigma_{n}=\sigma /\left(1+\beta^{2}\right)$. Since $E_{x}$ is not known a' priori, and because equation (13) is linear in $\varphi$, a decomposition of the potential is permitted as follows:

$$
\varphi(y, z)=E_{x} \varphi_{1}+\varphi_{2}
$$

The two functions, $\varphi_{1}$ and $\varphi_{2}$, are governed by the following equation set:

$$
L\left(\varphi_{1}\right)=\frac{\partial}{\partial y}\left[\left(\beta-\cot \theta_{d}\right) \sigma_{n}\right]
$$

and

$$
L\left(\varphi_{2}\right)=-\frac{\partial}{\partial y}\left[\sigma_{n} B(u-\beta v)\right]
$$

where the operator, $L$, is

$$
L=\frac{\partial}{\partial y}\left(\sigma_{n} \frac{\partial}{\partial y}\right)+\frac{\partial}{\partial z}\left(\sigma \frac{\partial}{\partial z}\right)
$$

Note that the function $\varphi_{1}$ is driven by temperature nonuniformities, while $\varphi_{2}$ is driven by temperature and velocity nonuniformities. Both functions are independent of $E_{x}$, which is reduced to a parameter of the problem to be determined later from the specification of the external electrical connection and loading. From Ohm's Law, the current density components $-J_{x}, J_{y}$, and $J_{z}-$ can be expressed as 


$$
\begin{gathered}
J_{x}=E_{x} \sigma_{n}\left(1+\beta \cot \theta_{d}+\beta \frac{\partial \varphi_{1}}{\partial y}\right)+\sigma_{n}\left[(\beta u+v) B+\beta \frac{\partial \varphi_{2}}{\partial y}\right], \\
J_{y}=E_{x} \sigma_{n}\left(\beta-\cot \theta_{d}-\frac{\partial \varphi_{1}}{\partial y}\right)-\sigma_{n}\left[(u-\beta v) B+\frac{\partial \varphi_{2}}{\partial y}\right],
\end{gathered}
$$

and

$$
J_{z}=-E_{x} \sigma \frac{\partial \varphi_{1}}{\partial z}-\sigma \frac{\partial \varphi_{2}}{\partial z} .
$$

To find the cross-sectional averages of $J_{x}$ and $J_{y}$, the cross-sectional spatial average must be defined as

$$
\langle f\rangle=\frac{1}{A} \int f(y, z) d A,
$$

where $A$ is the cross-sectional area normal to the channel, which yields the following average equations:

and

$$
\left\langle J_{x}\right\rangle=E_{x}\left[\left\langle\left(1+\beta \cot \theta_{d}\right) \sigma_{n}\right\rangle+\left\langle\sigma_{n} \beta \frac{\partial \varphi_{1}}{\partial y}\right\rangle\right]+\left\langle\sigma_{n} \beta \frac{\partial \varphi_{2}}{\partial y}\right\rangle+B\left\langle\sigma_{n}(\beta u+v)\right\rangle
$$

$$
\left\langle J_{y}\right\rangle=E_{x}\left[\left\langle\left(\beta-\cot \theta_{d}\right) \sigma_{n}\right\rangle-\left\langle\sigma_{n} \frac{\partial \varphi_{1}}{\partial y}\right\rangle\right]-\left\langle\sigma_{n} \frac{\partial \varphi_{2}}{\partial y}\right\rangle-B\left\langle\sigma_{n}(u-\beta v)\right\rangle .
$$

Applying the boundary conditions for diagonal insulating sidewall and diagonal conducting sidewall MHD generators, the axial electric field is

$$
E_{x}=\frac{J_{l d}-\left\langle\sigma_{n}\left(\beta+\cot \theta_{d}\right)\left(\varphi_{2 y}+u B\right)\right\rangle-B\left\langle\sigma_{n}\left(1-\beta \cot \theta_{d}\right) v\right\rangle}{\left(1+\cot ^{2} \theta_{d}\right)\left\langle\sigma_{n}\left(\beta+\cot \theta_{d}\right) \varphi_{1 y}\right\rangle} .
$$

\subsubsection{State Equations}

In order to close the system of fluid dynamic and electrical equations, it is necessary to establish a relationship between the fluid properties and the chosen solution transport variables $\left(u, v, w, p_{\text {avg }}, h\right)$. In the MGMHD code, electrical and thermodynamic properties are calculated from two independent thermodynamic variables, $\left(p_{\text {avg }}, h\right)$ or $\left(p_{\text {avg }}, T\right)$, depending on which of the four following options are chosen by the user:

(1) Constant fluid properties;

(2) Variable fluid properties with constant electrical properties;

(3) Variable fluid properties with $\sigma$ and $\beta$ calculated from empirical correlations; and, 
(4) Variable fluid and electrical properties calculated from fourth-order interpolation polynomials in $p_{\text {avg }}$ and $h$.

Each of the above-stated property options is detailed in the MGMHD user manual $;{ }^{3}$ however, it is important to note that all of the options use approximations and/or polynomial curve fits to generate the thermodynamic and electrical properties. The baseline MGMHD code does not include capability for generalized calculation of electrical transport properties in partially ionized gases.

\subsubsection{Required Modifications and Improvements}

The baseline MGMHD code is inadequate for three-dimensional modeling of MHD accelerators; however, it does fulfill some of the basic requirements and its structure and flexibility allow for the necessary modifications. As noted above, the PNS formulation of the MGMHD model enables threedimensional calculations to proceed without the penalty of slow convergence times or the need for massive computing resources. Moreover, the MGMHD code is mathematically structured for diagonal configurations, although the diagonalization angle must remain constant over the entire length of the MHD channel. Unfortunately, the thermodynamic and electrical conductivity submodels in the MGMHD code are, at best, generic curve fits, based on a few user-defined constants, but the code does allow the user to supply their own thermodynamic and electrical transport data via an input file.

In order to properly model MHD accelerators, the MGMHD code requires certain specific modifications, as follows:

- Structural modifications to permit both generator and accelerator calculations in Faraday, Hall, and diagonal configurations.

- Modifications to allow arbitrary variations in diagonalization angle along the channel length.

- Addition of thermodynamic and electrical transport property subroutines based on a generalized kinetics model applicable to arbitrary partially-ionized gas mixtures.

Though not necessarily required for the modeling of diagonal accelerators, there are several other desirable features that would enhance and improve the capabilities of the MGMHD code. These additional improvements are as follows:

- The addition of a realistic power-takeoff scheme at the entrance and exit of the simulated MHD channel which would allow for tailored distribution of current flow in these regions help and thereby avoid MHD compression effects at the inlet.

- Incorporation of accelerator efficiency calculations which would allow the user to more quickly determine optimum accelerator configurations.

- A three-dimensional calculation of total pressure as an aid to design optimization. 
- An axial current neutralized optimization option (an extension of the varying diagonal angle), which would facilitate determination of an optimum diagonalization angle and minimization of axial $\left(J_{x}\right)$ current flow.

\subsection{Extended Modeling Capabilities}

As noted above, the baseline MGMHD code was inadequate for proper modeling of diagonalized MHD accelerators, and significant modifications were required to correct these deficiencies. The structure of the modified numerical model, applicable to both MHD generators and accelerators in Faraday, Hall, and diagonal configurations, is referred to as the universal MGMHD model (UMM) and may be separated into two major blocks, as shown in figure 10. The thermoelectric block consists of the thermodynamic submodel (i.e., CEA routine), the electrical transport property submodel, along with library and input files that control the overall thermoelectric computation process. The MHD block consists of a modified version of the baseline MGMHD code.

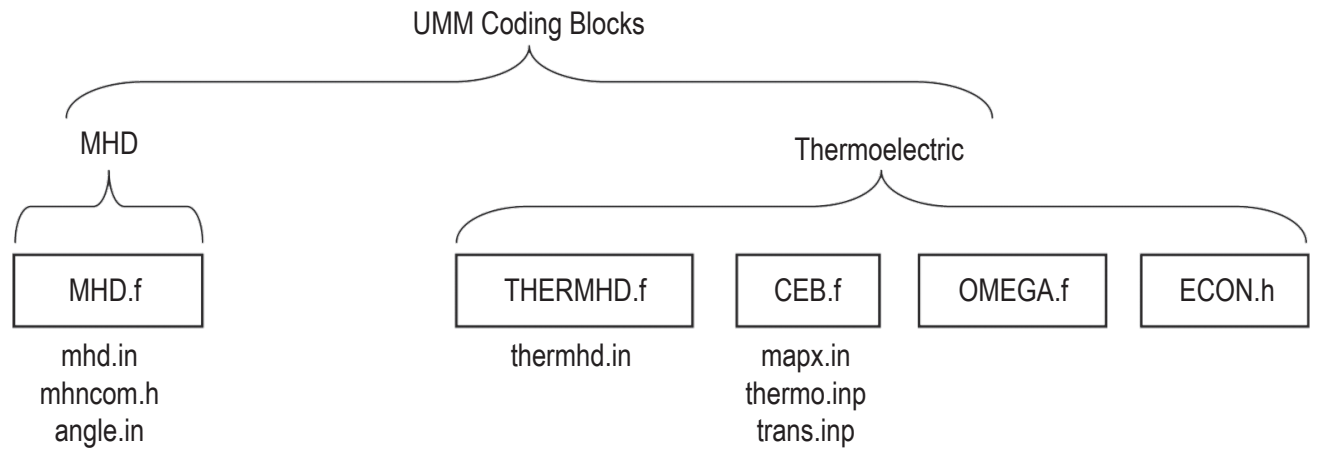

Figure 10. Structural coding blocks for the UMM three-dimensional numerical model.

\subsubsection{Thermodynamic and Electrical Transport Property Models}

The program thermhd.f manages the three routines named ceb.f, omega.f, and econ.h as shown in figure 10, where the ".f" extension denotes a Fortran file and the ".h" denotes an include file. The input file for thermhd.f is thermhd.in, in which the user defines the pressure and enthalpy ranges of interest and the desired tabulation intervals. The primary purpose of the thermoelectric codes is to create a lookup table of thermodynamic and electrical transport property data (temperature, molecular weight, ratio of specific heats, laminar viscosity, electrical conductivity, and total charge) that can be accessed by the MHD numerical block as it solves the coupled fluid dynamic and electromagnetic equations. The ceb.f routine has three input files: (1) thermo.inp, which automatically creates a library of thermodynamic data (no user input required), (2) trans.inp, which automatically creates a library of transport property data (also, no user input necessary), and (3) mapx.in, where the user defines the fluid species, weight percentages, and initial temperatures. The major difference between the ceb.f routine and the CEA code is that ceb.f has been modified to output an unformatted plot file of 39 variables representing the 9 thermodynamic variables and 30 gas species concentrations for which electron-momentum, cross-sectional data are available. It is important to note that all changes made to the CEA code deal with input/ouput (I/O) 
and formatting (i.e., none of the thermodynamic equations in the CEA code were altered). The ceb.f code runs at every step in the pressure and enthalpy looping process, as described in subsequent sections. For every step in the pressure and enthalpy loops, the ceb.f code provides the following information to the MHD numerical model:

- Temperature $(T)$.

- Molecular weight $(M W)$.

- Ratio of specific heats $(\gamma)$.

- Laminar viscosity $\left(\mu_{1}\right)$.

- Total charge $\left(e N_{e}\right)$.

In the UMM code, electrical conductivity is calculated in two parts, based on species collisions, using the omega.f and econ.h routines. Specifically, omega.f is a self-contained (i.e., no input file required) Fortran file that calculates electron-neutral collision integrals for 30 specified species using momentum-transfer collision cross-section data. ${ }^{11,12}$ Charged-particle collision integrals are then estimated in econ.h. All the collision integrals along with the species and total number densities mentioned earlier are then used by econ.h, which is an include file called from thermhd.f, to calculate the electrical conductivity of the gas mixture, based on a method described by Devoto, ${ }^{5}$ from the fundamental equation

$$
\sigma=\frac{e^{2} n}{\rho k T} \sum_{j=1}^{\varsigma} n_{j} m_{j} Z_{j} D_{1 j},
$$

where $e$ is the electron charge, $n$ is the total number density, $\rho$ is the total mass density, $n_{j}$ is the number density of the $j$ th species, $D_{1 j}$ is the multicomponent diffusion coefficient, and $j$ is the counter, with 1 representing electrons and 2 through $\zeta$ being ions (where $>2$ denoted multiple ionic states).

The transport properties and diffusion coefficients in the UMM model are derived from the collisional Boltzmann equation:

$$
\frac{\partial f}{\partial t}+\mathbf{v} \cdot \nabla f+\frac{\mathbf{F}}{m} \cdot \frac{\partial f}{\partial \mathbf{v}}=\left(\frac{\partial f}{\partial t}\right)_{c} .
$$

One solution to the Boltzmann equation is the Chapman-Enskog perturbation method (which is rigorously explained and expanded in Hirschfelder, Curtis, and Bird ${ }^{13}$ and Chapman and Cowling ${ }^{14}$ ) where one assumes that collisions are the driving mechanism for translational nonequilibrium of the gases and are such that the distribution functions of the various species differ only slightly from a Maxwellian distribution function. The Chapman-Enskog method uses a perturbation factor based on a scale length of macroscopic quantities and the mean free path between collisions. The distribution function is expanded, order by order, in the small perturbation parameter: ${ }^{14}$

$$
f(\mathbf{r}, \mathbf{u}, t)=f_{0}(\mathbf{r}, \mathbf{u}, t)+\varepsilon f_{1}(\mathbf{r}, \mathbf{u}, t)+\varepsilon^{2} f_{2}(\mathbf{r}, \mathbf{u}, t)+\cdots,
$$

where $f_{0}, f_{1}$, and $f_{2}$ are assumed to be of the same order of magnitude. If there are no gradients in the composition, velocity, and temperature in the gas, this reduces to a Maxwellian distribution: 


$$
f_{0}(\mathbf{r}, \mathbf{u}, t)=n(\mathbf{r})\left(\frac{m}{2 \pi k T}\right)^{3 / 2} \exp \left(-\frac{m \mathbf{u}}{2 k T}\right) .
$$

Typically, one is interested in the properties of gases that are under conditions only slightly different from equilibrium. In fact, it is only under these conditions that the flux vectors are linear in the derivatives and the usual definitions of the transport coefficients apply. ${ }^{13,14}$ In this limit, the distribution function is nearly Maxwellian, and the Boltzmann equation can be solved by the Chapman-Enskog perturbation method. The resulting solutions are then used to obtain expressions for the fluxes and the transport coefficients. ${ }^{13,14}$

For MHD channel flow, the following assumptions may be applied:

- Inelastic collisions are negligible.

- The number of ions and electrons are equal.

- There is only one user-defined ion species, which is singly ionized.

- The ion and electron temperatures are equal.

- The electron-heavy (i.e., electron-neutral, electron-ion) collision terms are neglected in deriving the expression for the ion and atom transport properties (i.e., electrons do not alter the direction, velocity, or momentum of heavy particles).

- The change in the heavy particle perturbation term during a collision will be neglected in obtaining the expressions for the electron transport properties (i.e., when an electron impacts a heavy particle, the perturbation to the heavy particle is insignificant when compared to the electron perturbation).

- The contribution of ions to the electrical conductivity equation has been neglected (which is consistent with the simplification introduced in solving the linearized electron Boltzmann equation).

Under these assumptions, the electron-neutral conductivity equation reduces to the form

$$
\sigma=\frac{e^{2} n_{1} n m_{1}}{\rho k T} D_{11}
$$

The solution of equation (27) for $f_{1}(\mathbf{r}, \mathbf{v}, t)$ is accomplished through an expansion of the function in a finite series of Sonine polynomials, ${ }^{5}$ with the level of approximation of the transport coefficients being the number of terms in the expansion series. It has been shown that at least the second-order approximation is required for a reasonable level of accuracy. (See ref. 15.) In the fourth-order approximation, the ordinary diffusion coefficient from equation (29) is given by

$$
\left[D_{11}\right]_{4}=\frac{3 n_{1} \rho}{2 n m_{1}} \frac{\sqrt{2 \pi k T / m_{1}}}{|q|}\left|\begin{array}{ccc}
q^{11} & q^{12} & q^{13} \\
q^{21} & q^{22} & q^{23} \\
q^{31} & q^{32} & q^{33}
\end{array}\right|
$$


where $|q|$ is the determinant of the $q^{m n}$ elements. The $q^{m n}$ elements are the coefficients to the simplified electron equilibrium Maxwellian distribution function and are dependent on $\bar{Q}_{i j}^{(l, s)}$, the Maxwellian velocity-averaged collision cross section between species $i$ and $j$. For example, one of the more simple equations for $q^{m n}$ is given by

$$
q^{11}=8 \sqrt{2} n_{1}^{2} \bar{Q}_{11}^{(2,2)}+8 \sum_{i} n_{1} n_{j}\left[\frac{25}{4} \bar{Q}_{1 j}^{(1,1)}-15 \bar{Q}_{1 j}^{(1,2)}+12 \bar{Q}_{1 j}^{(1,3)}\right],
$$

where the equations for $q^{m n}$ become more complex as the values of $m$ and $n$ increase. The Maxwellian velocity-averaged collision cross section can be calculated through the relation

$$
\bar{Q}_{i j}^{(l, s)}(T)=\frac{4(l+1)}{(2+1) !\left[2 l+1-(-1)^{l}\right]} \int_{0}^{\infty} \exp \left[-\delta^{2}\right] \delta^{2 s+3} Q_{i j}^{l}(g) d \delta .
$$

Here, $\delta^{2}=\mu_{i j} g^{2} / 2 k T$, where $\mu_{i j}$ is the reduced mass of the colliding species $i$ and $j$, and $g$ is the initial relative velocity before impact. Fortunately, it turns out that all of the $\bar{Q}_{i j}^{(l, s)}(T)$ terms from equation (32) with $l>1$ have coefficients proportional to a power of $m_{1} / m_{j}$ (where $1=$ electrons and $j \neq 1$ ), can be neglected. ${ }^{16}$ This leaves only values of $Q_{i j}^{1}$, which are the well-known momentum-transfer collision cross sections, which can be experimentally measured as a function of incident electron energy. ${ }^{11,12}$ The integral in equation (32) takes the form of a half-range Gauss-Hermite polynomial, which is a special case of a Gauss-Laguerre integral equation, and can be solved using a 16-point Gauss-Laguerre quadrature (which is done in omega.f), essentially calculating the values of the average collision cross section for the selected species. From these cross-sectional values, the electrical conductivity based on electron-neutral collisions can be calculated from equations (29)-(31). It is known that in some gases, the momentum-transfer approximation is not accurate to the $1 \%$ level and the angular distributions must be taken into account. For MHD applications, however, the use of momentum-transfer cross sections is adequate.

Because the ionization level in MHD devices is so low (say, on the order of $1 \%-2 \%$ ), electronneutral collisions obviously play the dominant role in determining the electrical conductivity of the fluid. However, for completeness, the UMM code has a conductivity model that incorporates charged-particle collisions into the global electrical conductivity of the flow. Charged-particle collision integrals are calculated using a method described by Zollweg, ${ }^{17}$ who modified the Spitzer formula to permit its application in calculating electrical conductivities of partially ionized gases in the ideal and nonideal plasma regions. Zollweg's modifications to the Spitzer formula result in the relation

$$
\bar{Q}_{i j}^{1} \cong 6 \pi \bar{b} \ln \left(1+1.4 \Lambda_{m}^{2}\right)^{1 / 2},
$$

which approximates the momentum-transfer cross section for charged-particle collisions. This result is then used in conjunction with equations (32) and (29) to determine the electrical conductivity due to charged-particle collisions. Note that $b$ is the averaged impact parameter and $A$ is the Coulombic logarithmic term. 


\subsubsection{Electrical Conductivity Sequence}

Electrical conductivity is calculated in the UMM code using the omega.f and econ.h routines. Specifically, equation (25) is used to sum the different contributions from the different species interactions. Equation (32) calculates a collision integral using two methods, depending on the species involved in the collision. Electron-neutral collisions are calculated using a 16-point Gauss-Laguerre quadrature, where the momentum-transfer cross-section data comes from experimental results for the 30 available species. ${ }^{11,12}$ Charged-particle collisions are approximated using the method devised by Zollweg, ${ }^{17}$ which is based on a modified Spitzer formula and assumes charge neutrality.

The calculation of the integral cross section for the charged-particle interactions is fairly straightforward where each cross section is calculated at the temperature of interest. However, the execution of the Gauss-Laguerre quadrature in omega.f is slightly more complex. Figure 11 is a flowchart representing the processes in omega.f, specifically, the approximations to the Chapman-Enskog method and the Gauss-Laguerre quadrature loop.

The electron momentum-transfer cross sections for the 30 species are given as a function of incident electron energy, in units of electronvolts. ${ }^{11,12}$ Using the Gauss-Laguerre quadrature for a given species, which uses abscissa and weight factors, represented by " $k$ " and " $m$ " loops, respectively, in figure 11, omega.f creates a table of nine "omegas" for each of the 801 temperatures of interest (from 1,000 to $9,000 \mathrm{~K}$ at $10 \mathrm{~K}$ intervals). The "omegas" physically represent the different $l$ and $s$ values of the average collision cross sections from equation (32). The program creates a separate file for each species, containing that specie's collision cross-section values, which are later used in the calculation of the $q^{m n}$ elements, such as in equation (31). For each specie cross-sectional table, linear interpolation is used to obtain cross sections for the parameters of the actual potentials (i.e., the actual temperatures of interest). The overall electrical conductivity calculation procedure is as follows: (1) omega.f calculates the "omega" integrals for electron-neutral collisions and creates the omega tables, (2) ceb.f calculates the thermodynamic data (e.g., temperature) and species concentrations (including ions), (3) econ.h calculates the "omega" integrals for charged-particle collisions and interpolates electron-neutral collision cross sections based on temperatures from ceb.f, and (4) econ.h calculates the total electrical conductivity, based on equation (25).

\subsubsection{Thermodynamic and Electrical Property Model Implementation}

The purpose of the UMM thermoelectric routines is to provide thermodynamic property data (temperature, molecular weight, the ratio of specific heats, laminar viscosity, and total charge) and electrical transport property data (electrical conductivity data) to the MHD numerical model. The thermoelectric codes accomplish this by creating a table that loops in pressure and enthalpy and contains the required thermoelectric data, as shown in figure 12.

As discussed in previous sections, the user specifies in thermhd.f the maximum and minimum pressure and enthalpy values, and the number of steps the code should take for the pressure and enthalpy loops. (Note that the code calculates each loop's step size by taking the difference between the maximum and minimum loop values and dividing that by the number of steps that should be taken. By making the step size a calculated value, the user is guaranteed that the thermoelectric codes will use their 


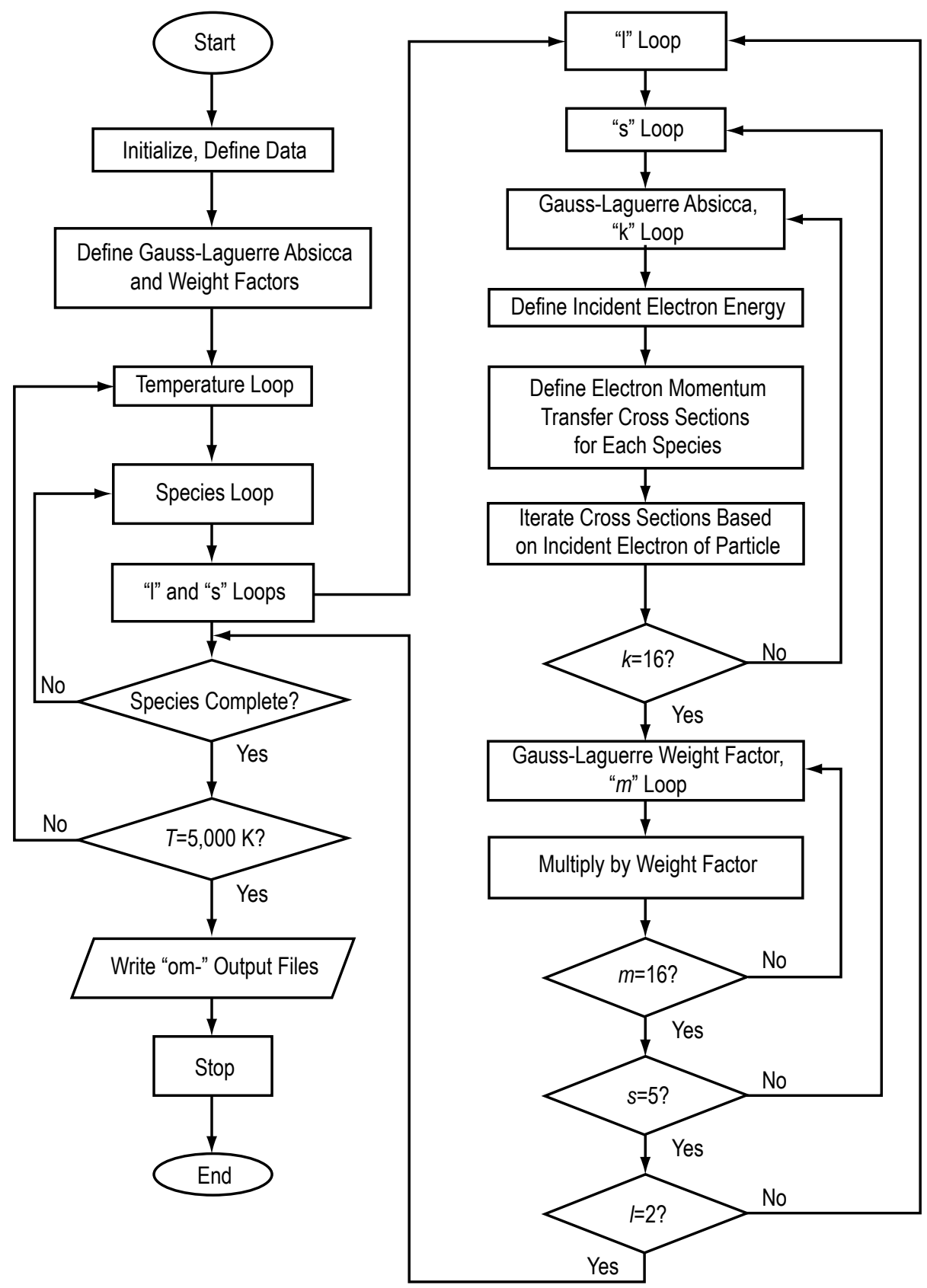

Figure 11. Flowchart of omega.f routine for execution of the Gauss-Laguerre quadrature procedure.

exact minimum and maximum values, and that the step sizes will be constant.) As equation (13) illustrates, for the first pressure step, the thermoelectric codes calculate the required properties at every step in the enthalpy loop. Then, the code advances to the second pressure step, calculates all the required values at every step in the enthalpy loop, again, and continues to advance in the same manner until both the enthalpy and pressure loops are completed. Currently, the maximum number of steps for pressure and enthalpy are 60 and 500, respectively; however, both values can be increased if necessary. 


$$
\begin{aligned}
& \text { OMEGA } \\
& P_{\text {min }}, P_{\text {max }}, \# P, h_{\text {min }}, h_{\text {max }}, \# \text { } \longrightarrow \text { THERMHD } \\
& \text { OX, Fuel, WT\%, } T_{\mathrm{OX}}, T_{\mathrm{F}} \longrightarrow \quad \mathrm{CEB} \\
& \text { ELCON } \\
& {\left[\begin{array}{cccccccc}
P_{01} & h_{01} & T_{1,1} & M W_{1,1} & \gamma_{1,1} & \mu_{1,1} & \sigma_{1,1} & \mathrm{eN}_{\mathrm{e}_{1,1}} \\
P_{01} & h_{02} & T_{1,2} & M W_{1,2} & \gamma_{1,2} & \mu_{1,2} & \sigma_{1,2} & \mathrm{eN}_{\mathrm{e}_{1,2}} \\
& \vdots & & & \vdots & \vdots & \vdots \\
P_{01} & h_{n} & T_{1, n} & M W_{1, n} & \gamma_{1, n} & \mu_{1, n} & \sigma_{1, n} & \mathrm{eN}_{\mathrm{e}_{1, n}} \\
\vdots & \vdots & \vdots & \vdots & \vdots & \vdots & \vdots & \vdots \\
P_{m} & h_{01} & T_{m, 1} & M W_{m, 1} & \gamma_{m, 1} & \mu_{m, 1} & \sigma_{m, 1} & \mathrm{eN}_{\mathrm{e}_{m, 1}} \\
\downarrow_{m} & h_{n} & T_{m, n} & M W_{m, n} & \gamma_{m, n} & \mu_{m, n} & \sigma_{m, n} & \mathrm{eN}_{\mathrm{e}_{m, n}}
\end{array}\right]}
\end{aligned}
$$

Figure 12. Structure of the thermoelectric property table created by the thermodynamic and electrical transport property submodels in the UMM code.

As previously stated, thermhd.f is the "managing" program of the thermoelectric routines. It initiates the thermodynamic and/or electrical transport property calculations, runs the main pressure and enthalpy loops, and controls most of the important I/O, including writing the thermoelectric data output file (illustrated in fig. 12) as required by the MHD block. Figure 13 is a flowchart representing the processes in thermhd.f, which includes the calculations made by econ.h and ceb.f.

\subsection{Universal MGMHD Model Modifications and Improvements}

This section details the modifications and additions made to the baseline MGMHD code. The resulting modified code, called mhd.f (with input files mhd.in, mhncom.h, and angle.in) defines the MHD block of the UMM code.

\subsubsection{Accelerator Mode}

For a given MHD channel, the primary difference between generator and accelerator modes is the direction of the $J_{y}$ current. The reversal of the $J_{y}$ current from generator mode to accelerator mode is typically accomplished with an applied current. That is, an external power supply is used to overpower the naturally occurring $J_{y}$ current, which stems from the $\mathbf{u} \times \mathbf{B}$ Lorentz force. This applied $J_{y}$ 


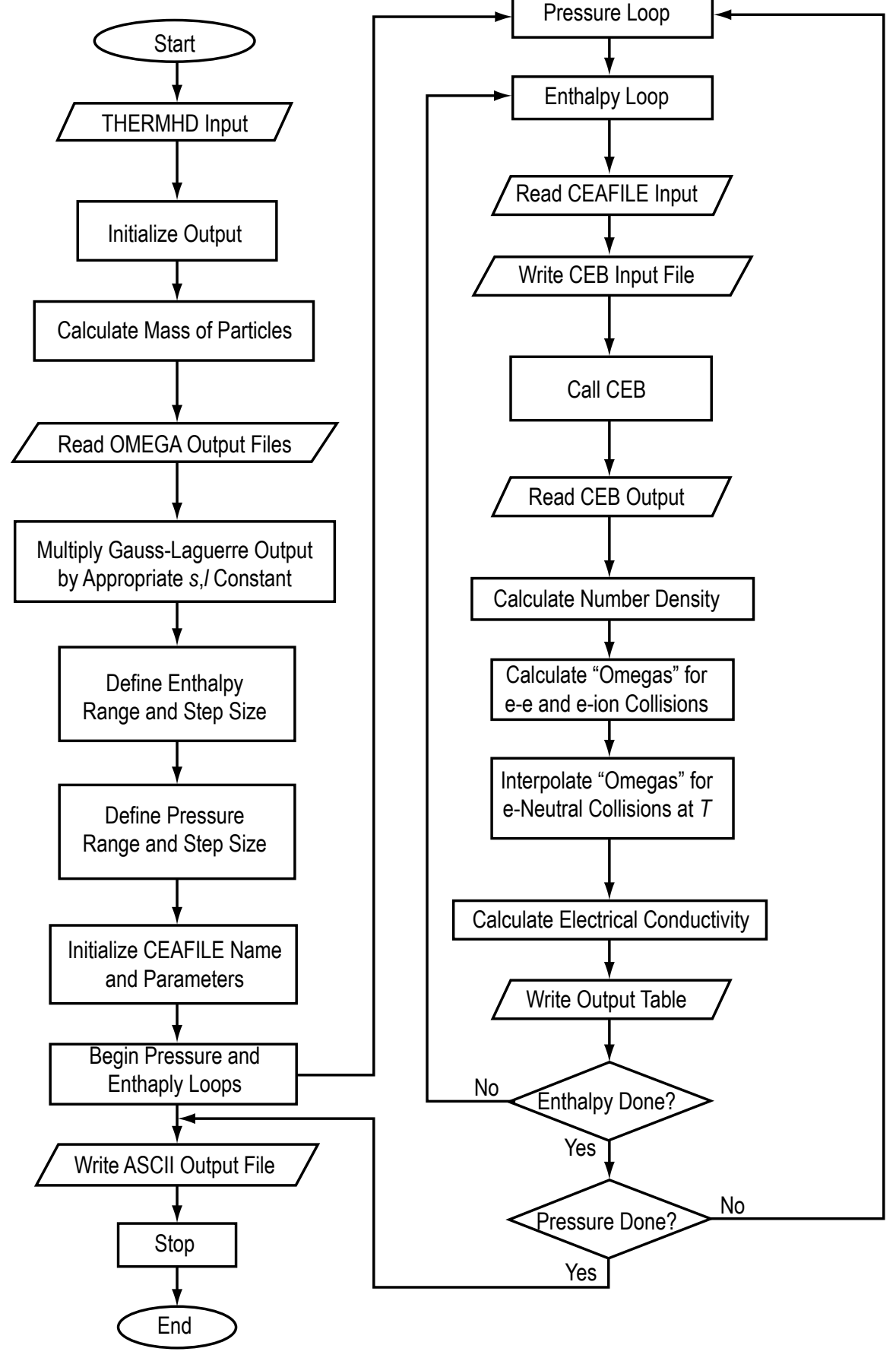

Figure 13. Flowchart for the thermhd.f program - includes the thermodynamic calculations made by ceb.f and the electrical calculations made by econ.h.

current results in a $J_{y} B$ Lorentz force that accelerates the flow in the positive $\mathbf{u}$ direction. Furthermore, in an MHD accelerator, since the user applies/specifies the $J_{v}$ current instead of one being induced by the interaction of the moving particles and the magnetic field, it can alter the solution procedure for the problem. 
The following sections explain how the MGMHD code was modified to achieve MHD accelerator operation in the respective configurations (Faraday, diagonal, and Hall). In all cases, even where potentials and currents are calculated in different ways, these modifications were additions to the MGMHD code and none of the original MGMHD functionality was lost. It is also important to note that when running the UMM code in any MHD accelerator configuration, the user should always doublecheck the output to ensure the proper orientation of the $J_{y}$ current. In accelerator mode, the $J_{y}$ current, under certain circumstances, sometimes reverses direction, essentially changing the accelerator to generator mode.

The primary goal of this research is to numerically model a diagonal MHD accelerator. Therefore, the majority of the modifications to the MGHMD code deal with facilitating the simulation of a diagonal accelerator. This section will focus on the modifications required to convert from a diagonal generator model to a diagonal accelerator model. For details of the other modifications and additions, including varying of the diagonal angle, axial current neutralized mode, power-takeoff, electrical efficiency, and three-dimensional total pressure, see the detailed descriptions in Turner. ${ }^{10}$

It is important to note that the governing equations and assumptions outlined in the "Baseline Modeling Capabilities" continue to hold. As explained in figures 9 and 10, the physical difference between a diagonal accelerator and generator is the diagonal link between the electrodes. Therefore, for an MHD accelerator, a new set of oblique coordinates must be defined, and for the purposes of this derivation, a new diagonal angle, $\alpha_{d}$, will be used. Using these new oblique coordinates and $\alpha_{d}$, it is possible to define the electric potential as

$$
\psi=-\left(x+y \cot \alpha_{d}\right) E_{x}+\varphi(y, z)
$$

where the relationship $\alpha_{d}$ and $\theta_{d}$ is as follows:

$$
\alpha_{d}=180-\theta_{d}
$$

Note that equation (12) is recovered when equation (35) is substituted into equation (34). Substituting equation (34) into equations (8)-(10) results in the following equation for the two-dimensional potential function:

$$
\frac{\partial}{\partial y}\left(\sigma_{n} \frac{\partial \varphi}{\partial y}\right)+\frac{\partial}{\partial z}\left(\sigma_{n} \frac{\partial \varphi}{\partial z}\right)=E_{x} \frac{\partial}{\partial y}\left[\left(\beta+\cot \alpha_{d}\right) \sigma_{n}\right]-\frac{\partial}{\partial y}\left[\sigma_{n} B(u-\beta v)\right],
$$

which differs from equation (13) only in the angle and the sign preceding the cotangent. Therefore, the same linear decomposition used on equation (13) can be applied to equation (36).

In the accelerator case as in the generator case, both $\varphi_{1}$ and $\varphi_{2}$ are independent of $E_{x}$, which is determined by the external connection and loading of the accelerator. Applying Ohm's Law, the current densities are as follows:

$$
J_{x}=E_{x} \sigma_{n}\left(1-\beta \cot \alpha_{d}+\beta \frac{\partial \varphi_{1}}{\partial y}\right)+\sigma_{n}\left[(\beta u+v) B+\beta \frac{\partial \varphi_{2}}{\partial y}\right]
$$




$$
J_{y}=E_{x} \sigma_{n}\left(\beta+\cot \alpha_{d}-\frac{\partial \varphi_{1}}{\partial y}\right)-\sigma_{n}\left[(u-\beta v) B+\frac{\partial \varphi_{2}}{\partial y}\right]
$$

and

$$
J_{z}=-E_{x} \sigma \frac{\partial \varphi_{1}}{\partial z}-\sigma \frac{\partial \varphi_{2}}{\partial z}
$$

where, again, the only differences from the generator equations are the angle and sign before the cotangent. The cross-sectional, spatial-averaged current densities are represented as follows:

$$
\left\langle J_{x}\right\rangle=E_{x}\left[\left\langle\left(1-\beta \cot \alpha_{d}\right) \sigma_{n}\right\rangle+\left\langle\sigma_{n} \beta \frac{\partial \varphi_{1}}{\partial y}\right\rangle\right]+\left\langle\sigma_{n} \beta \frac{\partial \varphi_{2}}{\partial y}\right\rangle+B\left\langle\sigma_{n}(\beta u+v)\right\rangle
$$

and

$$
\left\langle J_{y}\right\rangle=E_{x}\left[\left\langle\left(\beta+\cot \alpha_{d}\right) \sigma_{n}\right\rangle-\left\langle\sigma_{n} \frac{\partial \varphi_{1}}{\partial y}\right\rangle\right]-\left\langle\sigma_{n} \frac{\partial \varphi_{2}}{\partial y}\right\rangle-B\left\langle\sigma_{n}(u-\beta v)\right\rangle .
$$

For the boundary conditions of a diagonal connection, the axial electric field is determined by the following equation:

$$
E_{x}=\frac{J_{l d}-\left\langle\sigma_{n}\left(\beta-\cot \alpha_{d}\right)\left(\varphi_{2 y}+u B\right)\right\rangle-B\left\langle\sigma_{n}\left(1+\beta \cot \alpha_{d}\right) v\right\rangle}{\left(1+\cot ^{2} \alpha_{d}\right)\left\langle\sigma_{n}\left(\beta-\cot \alpha_{d}\right) \varphi_{1 y}\right\rangle} .
$$

The purpose of this brief derivation was to show the differences and similarities in the generator and accelerator cases. A quick comparison of equations (18) and (37), equations (19) and (38), equations (22) and (40), equations (23) and (41), and equations (24) and (42), respectively, shows that the only difference between the generator and accelerator cases is the sign preceding the cotangent of the angle. Here, it is important to recall a basic property of the cotangent. That is, the cotangent of an angle is equal to the negative value of the cotangent of the supplement of that angle. In other words, if one uses an obtuse diagonalization angle (if $\theta_{d}$ is $>180^{\circ}$ ), the result would be the same as if one used the angle $\alpha_{d}$, described in this section. Therefore, in order to modify the MGMHD code to run in accelerator mode, one needs only to define the diagonalization angle in terms of the obtuse angle, $\theta_{d}$. Thus, in the MHD block of the UMM numerical model (i.e., mhd.f), the baseline MGMHD code was altered to accept obtuse diagonalization angles, which the user inputs in mhd.in.

\subsubsection{Integration of Thermodynamic and Electrical Property Models}

Figure 13 shows the structure of the UMM thermoelectrical data table, while figure 14 illustrates how these thermoelectrical data are used by the MHD code in three-dimensional calculations. The UMM MHD block solves for pressure at each axial location using the value of static pressure at the previous axial station and the pressure gradient from the momentum equation, and solves for enthalpy in each 


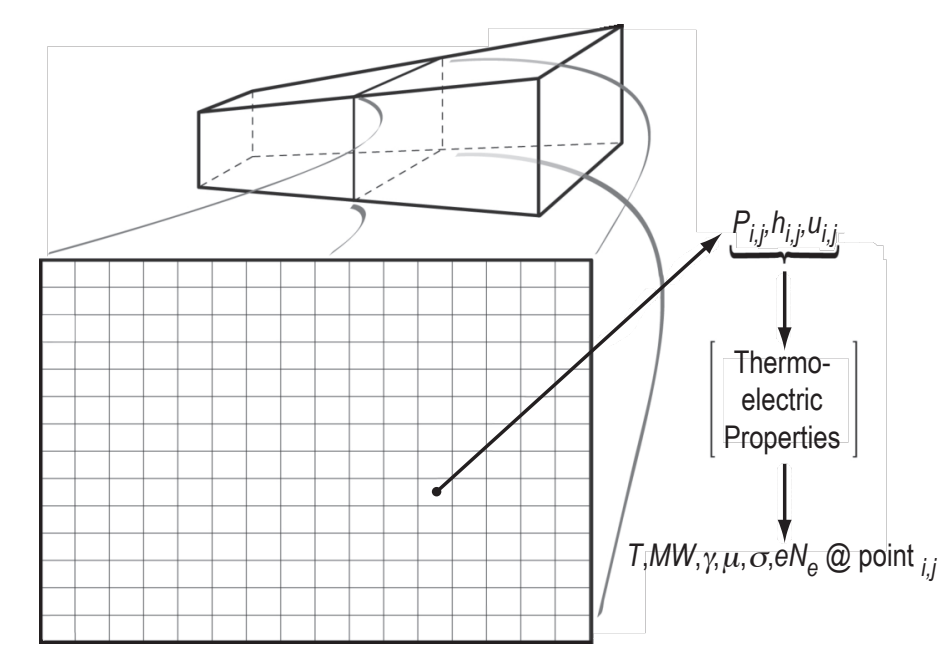

Figure 14. Illustration of UMM MHD block using the thermoelectrical data in three-dimensional calculations.

cell at each axial position using the energy equation. (See sec. 3.1.1, Fluid Dynamic Model). Similarly, using the momentum equation and mass continuity equation, the UMM MHD block solves for the value of axial velocity in each cell at each axial location. For each cell at each axial location, the code interpolates for the values of temperature $(T)$, molecular weight $(M W)$, ratio of specific heats $(\gamma)$, laminar viscosity $\left(\mu_{1}\right)$, electrical conductivity $(\sigma)$, and total charge $\left(e N_{e}\right)$ using the calculated values of pressure and enthalpy and the values of pressure and enthalpy from the thermoelectrical data tabulation. With this information and the aforementioned axial velocity measurements, virtually any required magnetohydrodynamic parameter (e.g., gas constant, sonic speed, Mach number, total pressure, and Hall parameter) may be calculated. Within the mhd.f program, tabular data look-up, two-dimensional interpolation, and pointwise calculation of thermoelectrical properties in the cross-sectional plane occur in different subroutines. 


\section{MAGNETOHYDRODYNAMIC AUGMENTED PROPULSION EXPERIMENT REGIME AND ANALYSIS PARAMETERS}

Because the point of focus of this research is directed at three-dimensional simulation of the MAPX accelerator, all other external factors are considered frozen for this analysis. In other words, all upstream conditions are considered to be constant as defined at the accelerator channel inlet. Therefore, all user-defined input parameters to the thermoelectric codes will remain constant in the following MAPX analyses. The pressure range, enthalpy range, working fluids, weight percentages, and inlet temperature are all predetermined by the arc-heater stagnation conditions and primary nozzle expansion characteristics. Therefore, the same thermoelectric data table was used for all MAPX performance analyses. This is also helpful in determining the causes of certain phenomena observed in the accelerator channel calculations. After a synopsis of the input and flow parameters, the results of an exploratory analysis over the trade-space were presented followed by detailed three-dimensional calculations for the selected design point.

\subsection{Input and Flow Parameters}

The thermodynamic flow parameters are presented in table 3. These values are input for the thermodynamic models (the modified CEA code, called ceb.f, and the managing thermodynamic file, thermhd.f). All of these values are held constant throughout the analysis described in this TM.

Table 3. MAPX defined thermodynamic parameters.

\begin{tabular}{lc}
\hline Working fluid, $\mathrm{N}_{2}$ & $100 \mathrm{wt} . \%$ \\
Working fluid temperature & $2,700 \mathrm{~K}$ \\
Seed, $\mathrm{Na}$ & $22 \mathrm{wt} . \%$ \\
Seed, $\mathrm{K}$ & $78 \mathrm{wt} . \%$ \\
Seed, NaK temperature & $300 \mathrm{~K}$ \\
Seeding percentage & $1 \%$ \\
Pressure range & $0.001-5 \mathrm{bar}$ \\
Pressure steps & 60 \\
Enthalpy range & $7.0 \times 10^{5}-3.5 \times 10^{7} \mathrm{~J} / \mathrm{kg}$ \\
Enthalpy steps & 500 \\
\hline
\end{tabular}

Table 4 shows the entrance input parameters as they are in the MHD input file. These parameters define the flow that enters the MAPX accelerator, and define some of the physical parameters and connections of the accelerator. For all analyses presented in this TM, these values were held constant. Note that "PTO steps" defines the number of staircase steps in the power-takeoff current distribution at the entrance of the MAPX channel. 
Table 4. MAPX defined input parameters to UMM.

\begin{tabular}{lc}
\hline Flow rate & $0.130 \mathrm{~kg} / \mathrm{s}$ \\
Flow temperature & $2,700 \mathrm{~K}$ \\
Wall temperature & $1,000 \mathrm{~K}$ \\
Static pressure & $3.24240 \times 10^{5} \mathrm{~Pa}$ \\
Velocity in y-direction & - \\
Velocity in z-direction & - \\
Wall roughness height & - \\
Axial pressure gradient & - \\
Electrode pitch & $0.015 \mathrm{~m}$ \\
PTO length (\% of total length) & $0.083 \%$ \\
PTO steps & 5 \\
\hline
\end{tabular}

Figure 15 shows the physical characteristics of the MAPX channel. These are the same values presented in table 1; however, figure 15 depicts the difference in the length between the actual powered length and the total channel length. Note that the powered length is $9 \mathrm{~cm}$ shorter than the total channel length, and the UMM code only models the powered region of the channel. It is also important to note that the MAPX channel begins and ends with an insulator. The magnetic field used in these analyses is presented in figure 7 .

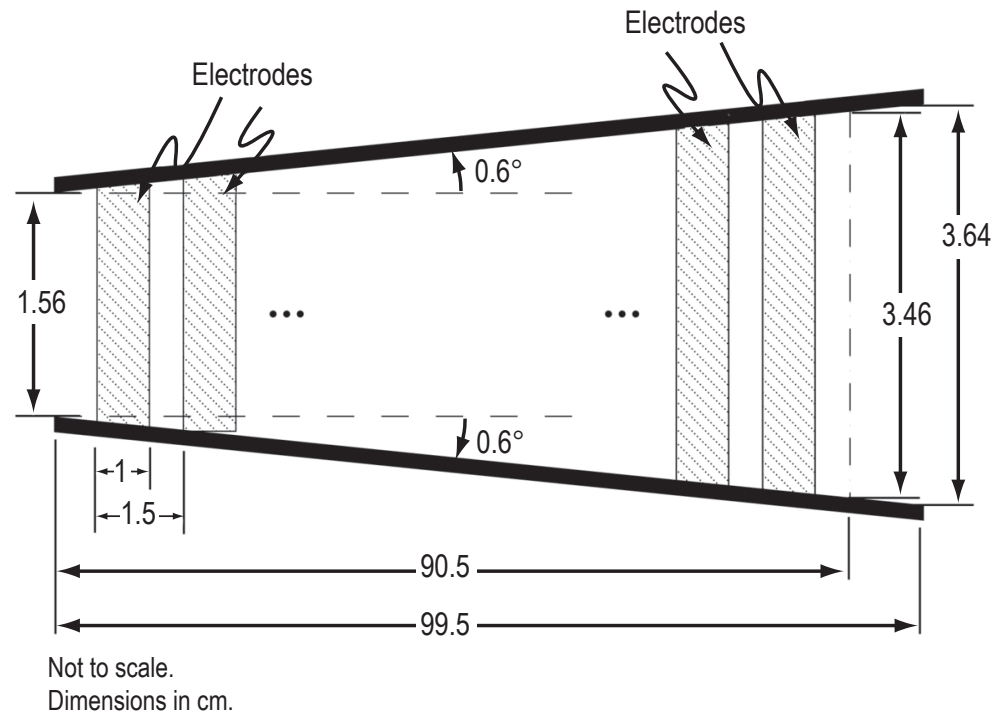

Figure 15. Physical characteristics of the MAPX accelerator.

\subsection{Thermodynamic/Electrophysical Regime}

Figure 16 shows some of the major parameters over the regime of interest to the MAPX accelerator. Please note that in order to see details in each plot, the axes are not oriented in the same direction. 

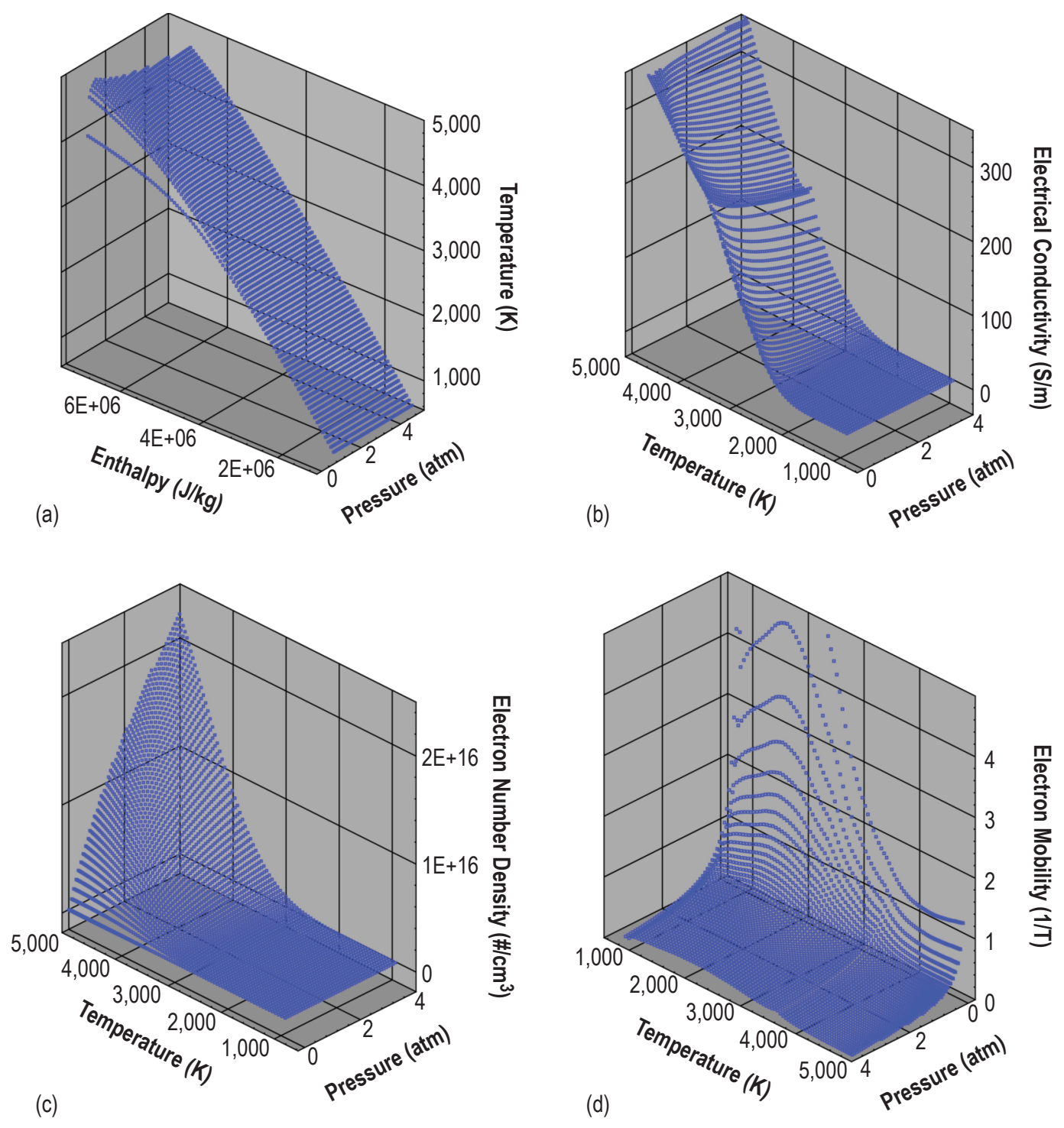

Figure 16. Major thermodynamic/thermoelectric parameters over regime of interest: (a) Temperature as a function of pressure and enthalpy, (b) electrical conductivity as a function of temperature and pressure, (c) electron number density as a function of temperature and pressure, and (d) electron mobility as a function of temperature and pressure.

From figure 16, temperature increases with increasing enthalpy, but seems to remain nearly constant for a given enthalpy with increasing pressure. The same is true for electrical conductivity - as temperature increases, conductivity also increases (after temperature reaches $\approx 2,500 \mathrm{~K}$ ). However, as pressure increases, this seems to cause the conductivity to decrease slightly, for a given temperature. This is logical—increasing pressure would inhibit electron movement and therefore conductivity. In part (c), electron number density increases with increasing temperature (after 3,000 K) and pressure. This is also logical - increasing temperature releases more electrons, and increasing pressure adds more particles per unit area. Electron mobility also seems to increase with temperature, but decrease with increasing pressure. 


\section{PARAMETRIC TRADE STUDY AND DESIGN OPTIMIZATION ANALYSES}

In the parametric analysis of the trade space, applied currents from zero through $300 \mathrm{~A}(25-\mathrm{A}$ increments) were tested against different diagonal angles ranging from $0^{\circ}$ to $60^{\circ}$, as measured from the positive $y$-axis. In addition to this diagonal angle trade study, the UMM code was also run in "axial current neutralized mode," which, in principle, should provide a reliable indication of the optimum angle for superior performance given a specific channel configuration. Since only the $J_{y} B$ Lorentz force pushes the flow downstream, axial current provides no positive acceleration effect and therefore reduces overall performance. Hence, the axial current neutralized mode, which uses the cross-sectional averaged current values, yields an estimate of the optimum diagonalization angle for best performance.

Figure 17 shows the axial variation of diagonalization angle, $\theta_{d}$, for axial current neutralized case with 100-A applied current. According to this figure, the optimum diagonalization angle $\left(\theta_{d}\right.$, as measured from the positive $x$-axis) is between $140^{\circ}$ and $150^{\circ}$ over most of the channel. However, due to physical constraints, a $135^{\circ}$ diagonalization angle is the maximum that can be practically attained in the accelerator, and is therefore chosen for the remainder of the parametric analyses in this TM.

It is important to how the diagonalization angle is defined. In this case, $\theta_{d}$ is measured from the positive $x$-axis and is always obtuse in accelerator mode. However, the diagonalization configuration may also be adequately defined with reference to the electric field angle, $\theta$, which is measured from the positive $y$-axis. This angle is always an acute value (for generators and accelerators), and one must simply know whether a generator or accelerator is being discussed. Therefore, the 135 diagonalization angle $\left(\theta_{d}\right)$ is equivalent to a 45 electric field angle $(\theta)$.

Figure 18 shows the plots of cross-sectional averaged (a) velocity and (b) temperature as a function of downstream axial location in the MAPX accelerator. The different lines and numbers on each plot represent different levels of applied current (zero to $300 \mathrm{~A}$, where the "hydro" case has no applied current and no magnetic field). From figure 18(a), it is observed that the highest levels of applied current do not result in the highest cross-sectional averaged axial velocities. This is due, in large part, to the excessive joule heating under high current loading conditions, as indicated in figure 18(b). Specifically, the highest cross-sectional averaged velocity occurs in the 125-A case. Higher current levels show reduced axial velocities. Part (b) shows that both the 125- and 100-A applied current cases have very stable cross-sectional temperatures. After some initial heating, they remain constant through the remainder of the channel. The highest levels of applied current show excessive and runaway heating and detrimental performance effects. Also note that in some cases (e.g., 300 A), the UMM code terminated before reaching the end of the channel due to the occurrence of flow reversal, which violates the PNS approximation.

Figure 19 shows the centerline total pressure as a function of the downstream axial distance at various levels of applied current. The flow enters the channel with a total pressure of $\approx 8.4 \mathrm{~atm}$. Depending on the level of applied current, the flow exits the accelerator channel with a net gain or loss in total pressure. 


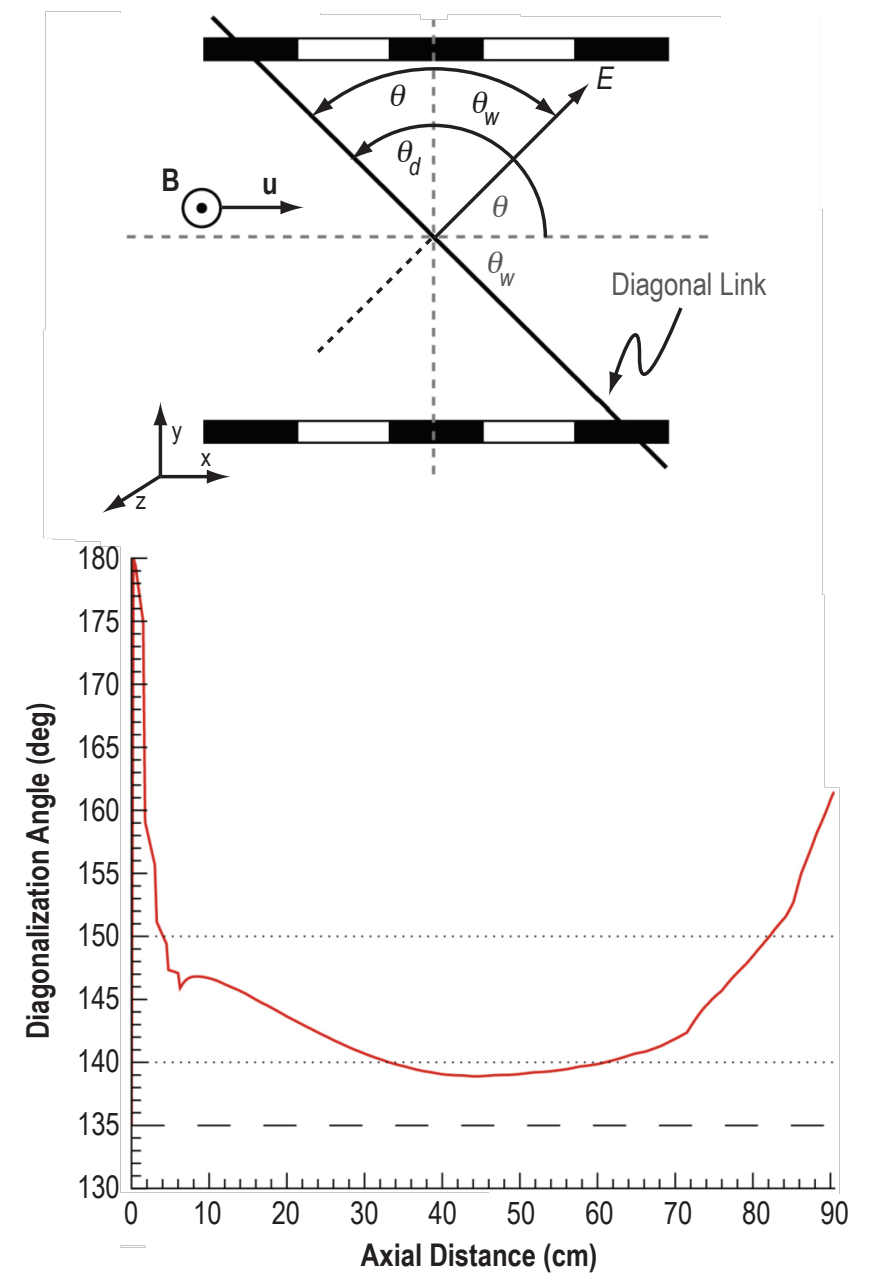

Figure 17. Axial variation of diagonalization angle, $\theta_{d}$, for axial current neutralized case with 100-A applied current. Definition of $\theta$ and $\theta_{d}$ illustrated above.

Observed was that the highest levels of applied current (175 to $300 \mathrm{~A}$ ) experience the severest losses in total pressure. This is consistent with figure 18(a) in which the highest levels of applied current cause axial velocity deceleration in the second half of the channel. Furthermore, all levels of applied current experience some form of total pressure loss in the channel. In the 100-A case, this total pressure loss occurs $\approx 75 \mathrm{~cm}$ downstream, suggesting that frictional forces begin to overpower the flow at this location. As seen in figure 18(a), the slope of the axial velocity curve also begins to flatten out at this location, suggesting that the decrease in axial acceleration is related to the decrease in total pressure.

The cross-sectional averaged $J_{y}$ current density is shown in figure 20 at various levels of applied current. Note that the stair-step distribution at the entrance to the channel is associated with the powertakeoff region. Of particular relevance, however, is the fact that $J_{y}$ tends to decrease down the channel. This is a critical effect since it is $J_{y}$ that controls the magnitude of the $\mathbf{J} \times \mathbf{B}$ Lorentz force and thereby determines acceleration effectiveness. The resulting $J_{y}$ current arises from the net electric field, which is the difference between the induced electric field $(\mathbf{u} \times \mathbf{B})$ and the externally applied field. Therefore, as 

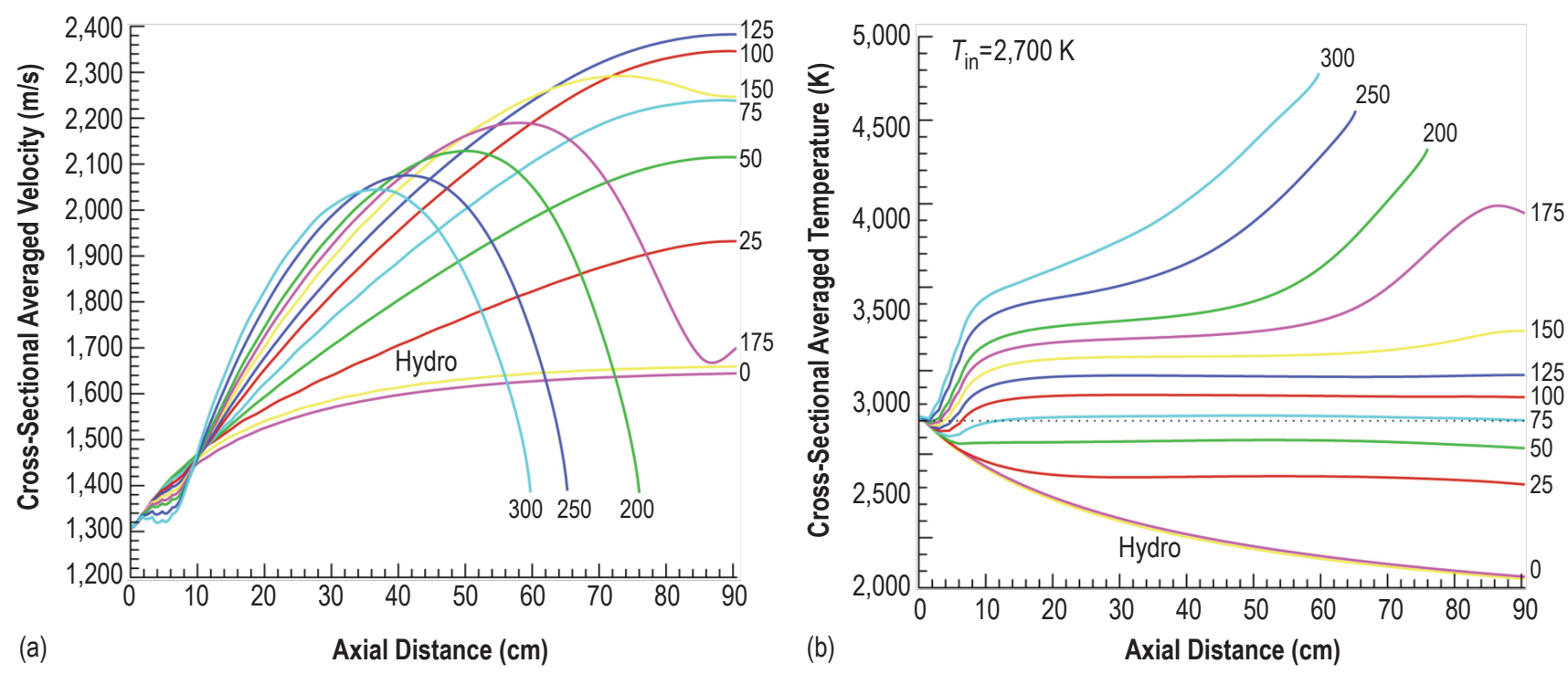

Figure 18. Cross-sectional averaged (a) velocity and (b) temperature as a function of axial distance in the MAPX accelerator. Levels of applied current are indicated.

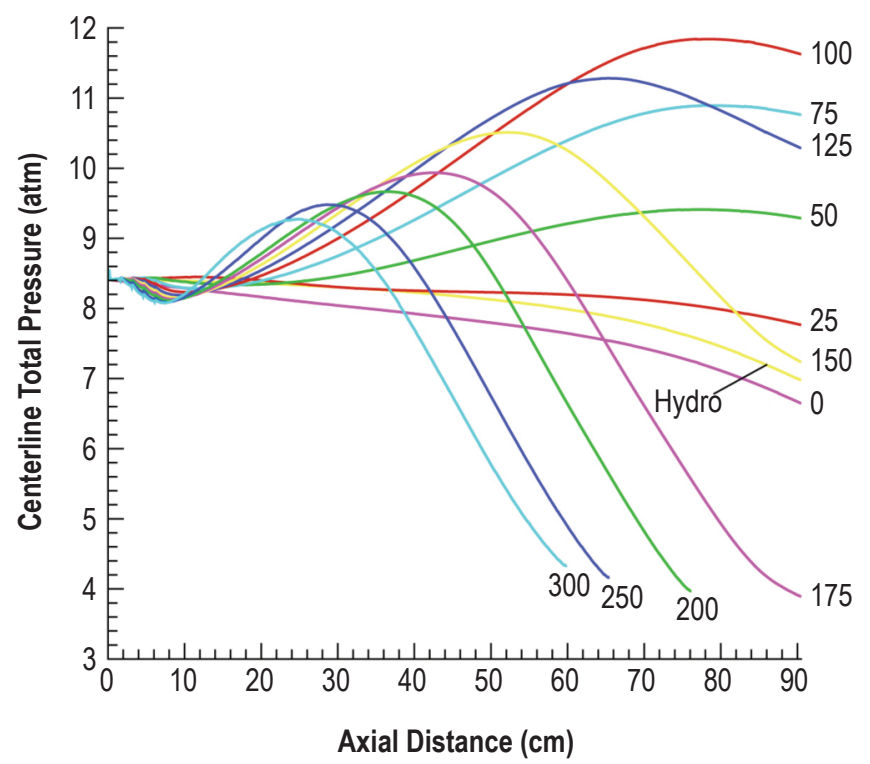

Figure 19. Centerline total pressure as a function of axial position. Levels of applied current are indicated.

flow velocity increases (as indicated in fig. 18), the induced electric field increases, yielding an overall net reduction in the transverse electric field. The net decrease in the transverse electric field therefore results in a net decrease in the transverse current, although total current is conserved. It is also important to realize that $J_{y}$ is a measure of current density, and as the cross-sectional area of the channel increases, the current density will naturally decrease, while the velocity will naturally increase due to supersonic 


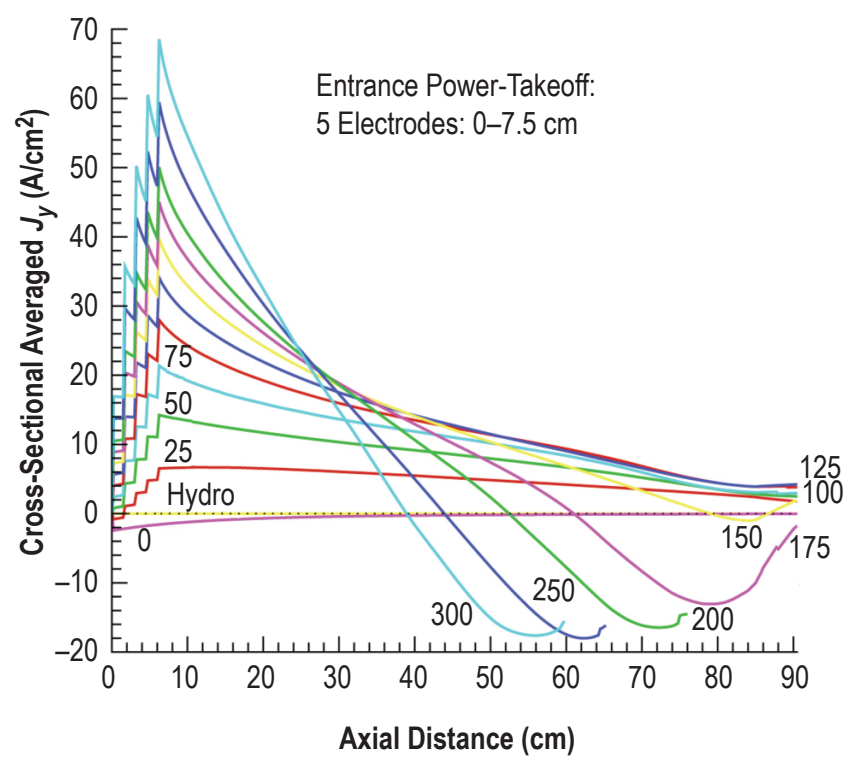

Figure 20. Cross-sectional averaged transverse current density, $J_{y}$, as a function of axial position. Levels of applied current are indicated.

expansion. Based on the results presented in figure 20, the 100- and 120-A applied current cases maintain the highest levels of $J_{y}$ current density throughout most of the MAPX channel, and are therefore near optimum from a performance perspective.

Figure 21 shows the axial current density, $J_{x}$, as a function of axial distance-part (a) showing the cross-sectional average $J_{x}$ values and part (b) giving the centerline values for $J_{x}$. The differences between the cross-sectional averaged and centerline axial current densities are significant. The crosssectional averaged current densities are all $<0.05 \mathrm{~A} / \mathrm{cm}^{2}$, while the centerline values for $J_{x}$ are as high as $70 \mathrm{~A} / \mathrm{cm}^{2}$. The large centerline values coupled with the very small cross-sectional averaged values indicate that within each cross-sectional plane, there exist upstream and downstream axial currents of approximately the same magnitude.

Recall that the axial current neutralized mode uses the cross-sectional averaged values of $J_{x}$ to calculate the appropriate angle. Since the diagonal angle is applied across the entire cross section, it is appropriate for the algorithm to use the cross-sectional values of $J_{x}$. The nonzero values for the crosssectional averaged $J_{x}$, as indicated in figure 21 , are a result of the physical limitations of the diagonal angle configuration. Because the recommended axial current neutralized angle of $55^{\circ}$ is not physically practical, a $45^{\circ}$ angle was selected for evaluation, hence, the observance of small but finite crosssectional averaged $J_{x}$ values. The axial current neutralized mode does, however, allow for localized nonzero values of $J_{x}$, and the local values can be quite large as shown in figure 21 .

Power levels are also of great importance when designing and optimizing an MHD accelerator. Figure 22(a) shows the total cumulative power that the MHD accelerator delivers to flow at various levels of applied current. Note that the pure hydrodynamic and unpowered cases show no power delivered by the MHD channel. Also note that, for the cases that did not terminate prematurely, there is a 

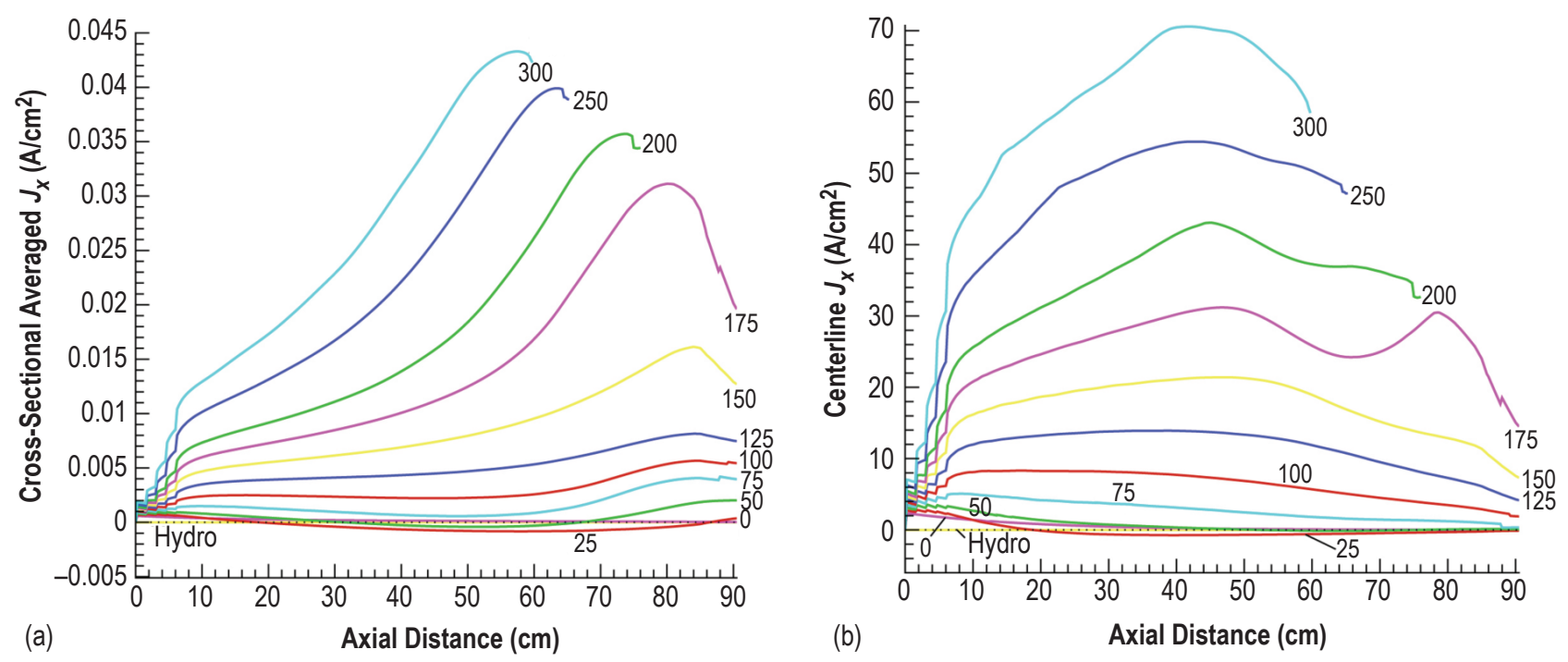

Figure 21. Axial current density, $J_{x}$, as a function of axial position: (a) Cross-sectional averaged $J_{x}$ and (b) centerline $J_{x}$. Levels of applied current are indicated.

diminishing rate of return for increasing levels of applied current. For example, between 75 and $100 \mathrm{~A}$, the difference in total cumulative power is $68 \mathrm{~kW}$; whereas, between 100 and $125 \mathrm{~A}$, the difference in total cumulative power is $\approx 45 \mathrm{~kW}$. As can easily be seen on the plot, the increase in total power diminishes for each additional 25-A increment for those cases for which the calculation did not terminate before the end of the channel. Since this graph shows a rate of diminishing total power with increased applied current, one would assume that there is an optimum level of applied current, above which losses would dominate.

The total cumulative power delivered to the flow, as shown in figure 22(a), either serves to accelerate the flow or heat the flow. Figure 22(b) shows the amount of total power delivered to the flow that was used to accelerate, or actually "push" the flow, as accumulated over the length of the MAPX accelerator. This graph shows the same diminishing returns on push power as were seen for total power; however, in this case, the 150- and 175-A cases show that push power drops off in the downstream region. Note, however, that between 75 and $100 \mathrm{~A}$, the difference in push power is $\approx 36 \mathrm{~kW}$, while the difference in push power between 100 and $125 \mathrm{~A}$ is only $\approx 15 \mathrm{~kW}$. So, although the 125 -A level of applied current does represent the highest level of accumulated push power, the difference from the previous level of applied current is less. It is also interesting to note that the unpowered case shows a small, but negative, push power. This is consistent with having no applied current, but allowing the magnetic field to induce a small, internal negative $J_{y}$ current, even though this is an open-circuit case.

Aside from acceleration, the total applied power can serve to heat the flow through joule heating or joule dissipation. Figure 22(c) shows the amount of the total power that was used to heat the flow providing no propulsive benefits whatsoever. The graph shows a similar "diminishing returns" phenomenon as seen in parts (a) and (b); however, the 150- and 175-A cases show enormous, increasing jumps in dissipated heat losses. Furthermore, the difference between the 75- and 100-A cases and between the 100 - and 125-A cases is almost negligible. Between 75 and $100 \mathrm{~A}$, the difference is $\approx 32 \mathrm{~kW}$, while the 

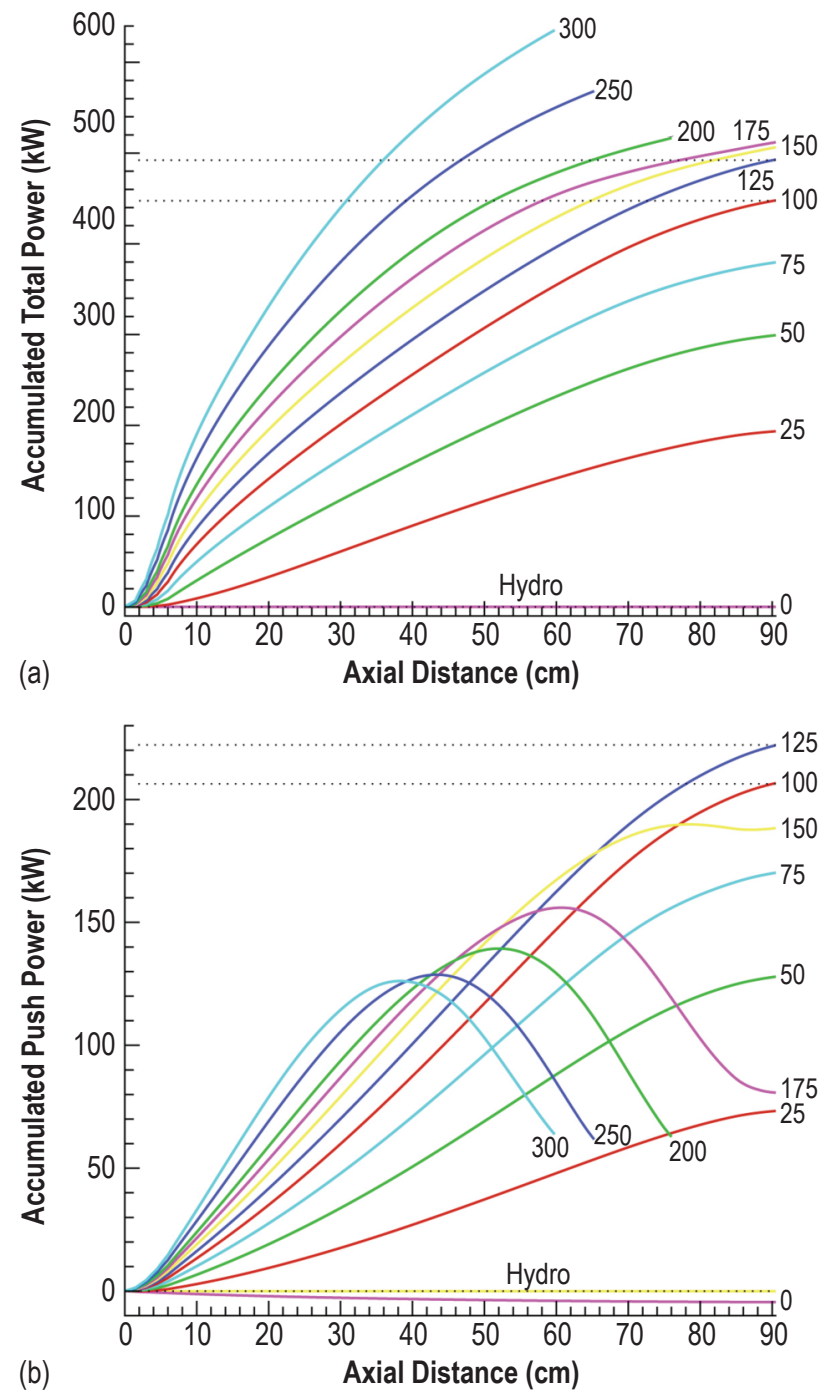

(b)

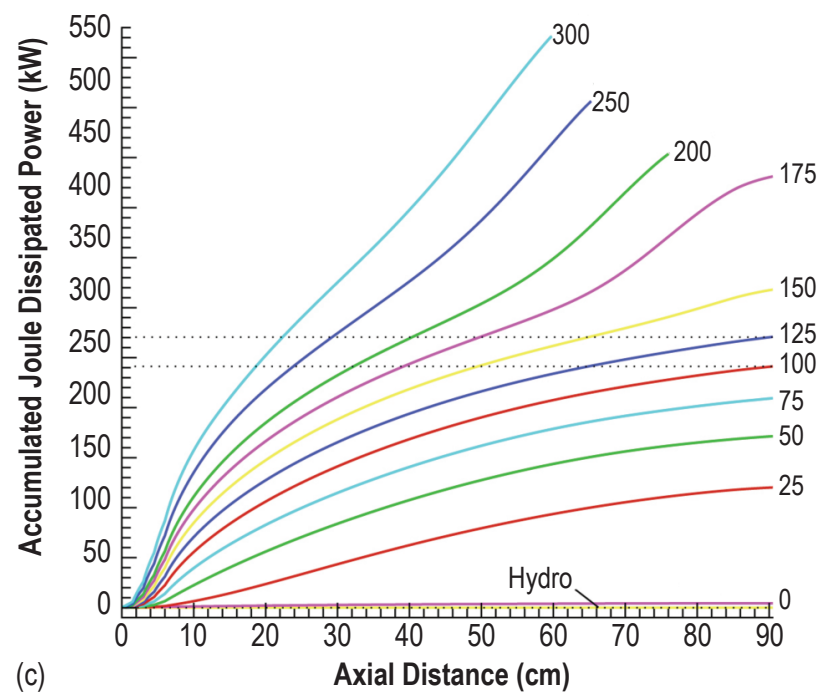

Figure 22. Various power levels along the channel: (a) Accumulated total power, (b) push power, and (c) joule dissipated power. 
difference between the 100 - and 125 -A cases is $\approx 30 \mathrm{~kW}$. Also note that the unpowered case shows a small, positive joule dissipation loss equal and opposite to the push power in (b), thereby yielding a zero summation.

Figure 22 tells an important story. While it is true that the 125-A case has the highest accumulated push-power level, one should note that only $15 \mathrm{~kW}$ of the $45 \mathrm{~kW}$ added total power (above the 100-A case) went toward flow acceleration. The other $30 \mathrm{~kW}$, twice the push-power value, went directly into joule dissipation losses (i.e., heating). Thus, only one-third of the added total power between 100 and $125 \mathrm{~A}$ was used to accelerate the flow at the 125 -A level. Moreover, $\approx 52 \%$ of the added total power between the 75- and 100-A current levels was used for acceleration in the 100-A case. Although the 125-A case does give higher total and push-power values, more of the additional applied power (above the $100-A$ case) is used to heat the flow rather than to accelerate the flow.

The differences in the push and applied power levels can best be explained through the global electrical efficiency, shown in figure 23. This graph clearly shows that, from a standpoint of power utilization, the 100-A case is superior to the 125-A case. Furthermore, since the 75- and 125-A cases are so near to each other (and both approximately equidistance from the 100-A case), the 100-A case seems to represent the highest electrical efficiency (i.e., 46\%) for the MAPX accelerator configuration. It should also be noted that the initial decrease in global efficiency in figure 23 is due to the low current levels in the power-takeoff region. In short, for an MHD accelerator used as an onboard propulsive device, efficiency is paramount, and the 100-A level of applied current yields the best efficiency.

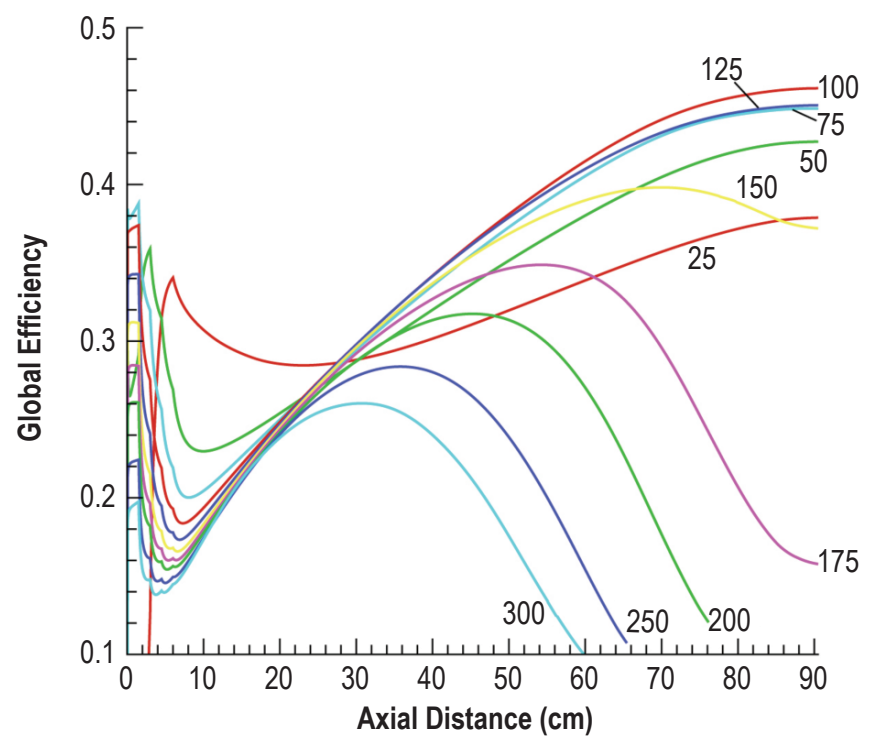

Figure 23. Cumulative global efficiency for the MAPX accelerator. Levels of applied current are indicated. 


\section{PRETEST DESIGN POINT ANALYSIS}

The optimal MAPX design point, as predicted by the UMM numerical model, occurs near 100-A applied current when diagonally connected with $\theta=45^{\circ}\left(\theta_{d}=135^{\circ}\right)$. Under these conditions, the crosssectional averaged velocity should almost double, the centerline total pressure should increase by $\approx 33 \%$, and the cross-sectional averaged temperature should remain approximately constant following a slight initial increase. The overall accelerator efficiency is $\approx 46 \%$. This section provides a detailed discussion of the three-dimensional simulation for this optimized design point.

Figure 24 shows the three-dimensional axial velocity at various downstream locations. The UMM code treats the flow at the entrance of the MAPX channel like a plug flow; therefore, the plot at $0.2 \mathrm{~cm}$ is still plug shaped. As the flow develops downstream, however, one notices that the bulk of the flow is attracted to the bottom wall (i.e., the electron accepting electrode surface). Therefore, the bulk of the flow seems to be pushed in the direction of the transverse electron flow.

Figure 25 shows a contour plot of the axial velocity, with a vector overlay representing the secondary flow velocity components (i.e., $v$ and $w$ ). MHD effects tend to increase the amount of secondary flow in a channel, as evident in figure 25. Generally, the secondary flow seems to be driving the axial core flow toward the bottom electrode. The center of the secondary flow, which appears to feed off the high-velocity regions in the primary axial flows, moves downward in the channel as the flow develops downstream. This behavior has been previously observed in MHD channels. ${ }^{18}$ The upward-directed secondary flow regions seem to be pushing the axial flow into the upper corners of the cross section, while simultaneously pushing the flow away from the center of the top electrode. This cross-plane circulation causes a concave region to form at the top of the axial profile, which, if allowed to develop further, might cause a separation in the core flow field.

Figure 18 shows that, following an initial increase, the cross-sectional averaged temperature profile for the 100-A case remained fairly constant. Figure 26, however, tells a different story. Figure 26 shows that, while the cross-sectional averaged temperature may be constant, there are large fluctuations in the temperature profile as the flow moves downstream. Most noticeable is the hot spot that begins to develop along the bottom electrode (i.e., the electron accepting electrode surface). This high-temperature region also corresponds with the downward skewing of the velocity profile and the strong secondary flow effects.

Figure 19 demonstrated that the centerline total pressure increased by $\approx 30 \%$ above the inlet condition. However, figure 27 shows that, while centerline total pressure does increase, there are severe viscous losses near the channel sidewalls. The flow is forced to enter as a plug, but sidewall shear quickly acts to retard the near-wall flow, thereby degrading total pressure in the region. Near the exit of the channel, the viscous losses from the sidewalls have actually started to encroach into the central region of the channel, limiting total pressure gains to a small region near the centerline. 


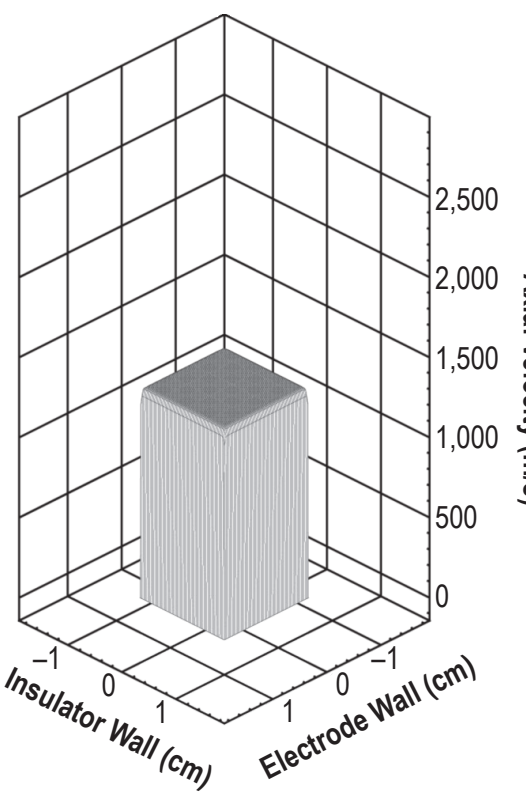

(a) $0.2 \mathrm{~cm}$

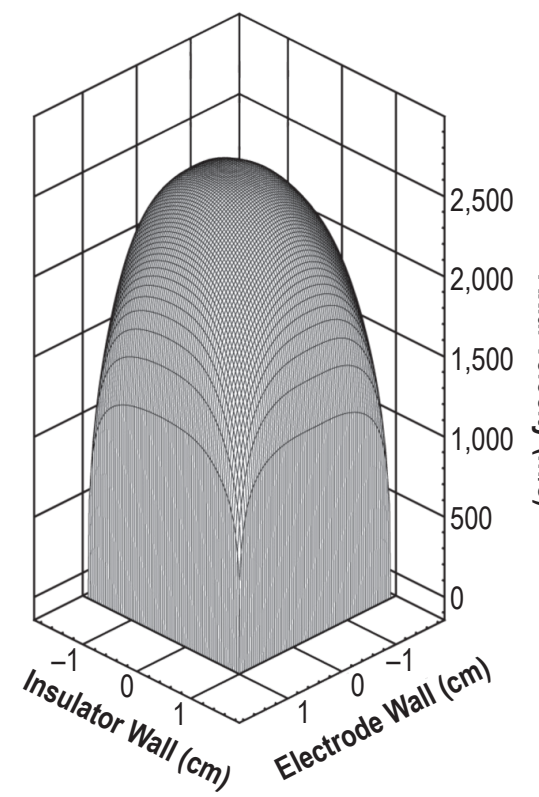

(d)

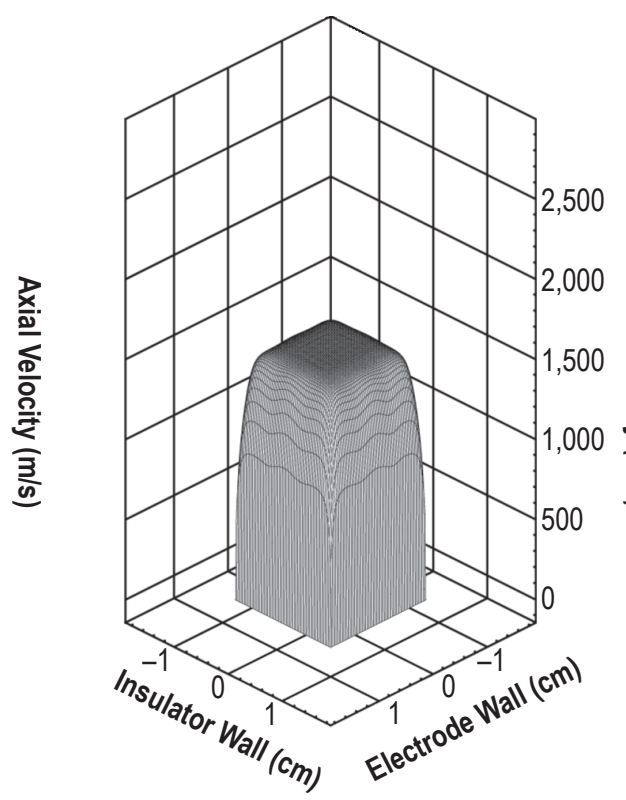

(b) $10 \mathrm{~cm}$

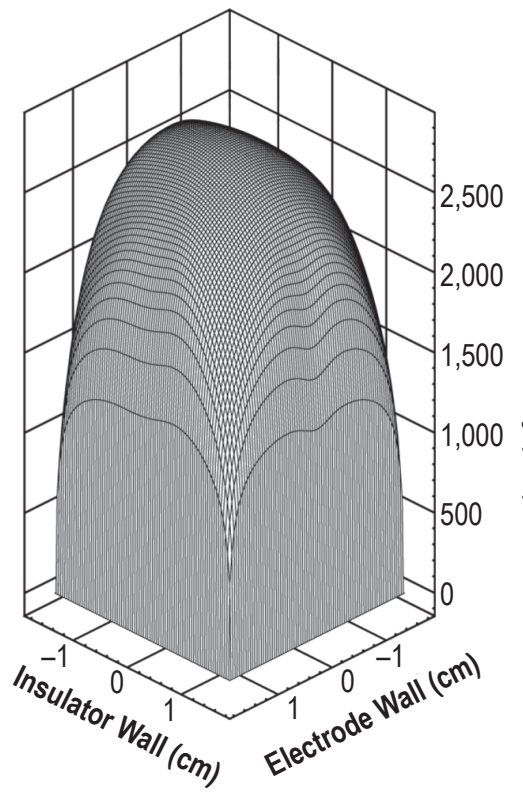

(e) $80 \mathrm{~cm}$

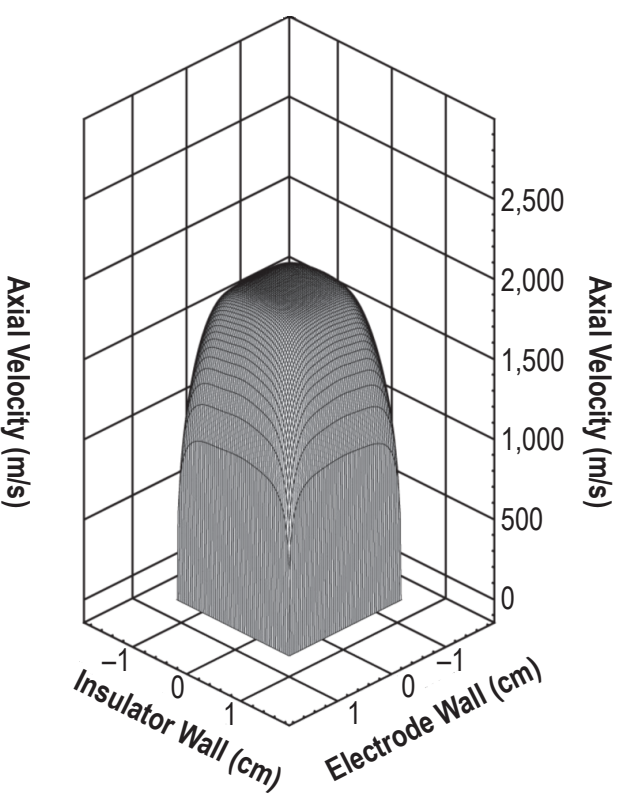

(c) $25 \mathrm{~cm}$

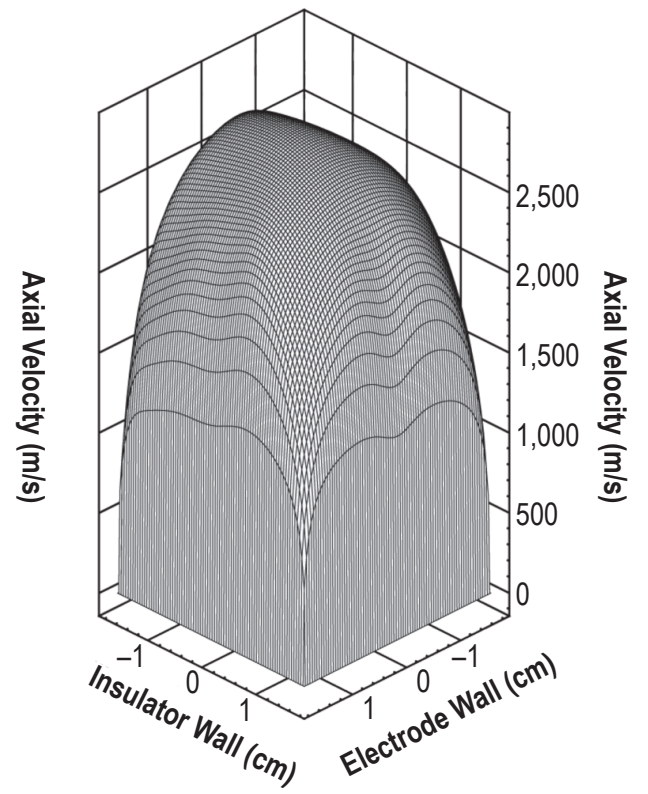

(f) $\quad 90.5 \mathrm{~cm}$

Figure 24. Three-dimensional axial velocity, $u$, plotted at (a) 0.2, (b) 10, (c) 25, (d) 60, (e)80, and (f) $90.5 \mathrm{~cm}$ along the MAPX accelerator channel. The last position corresponds to the end of the powered region in the channel. The positive $J_{y}$ current density flows from the bottom electrode (background) to the top electrode (foreground) in a direction out of the page. All velocities are in $\mathrm{m} / \mathrm{s}$. 

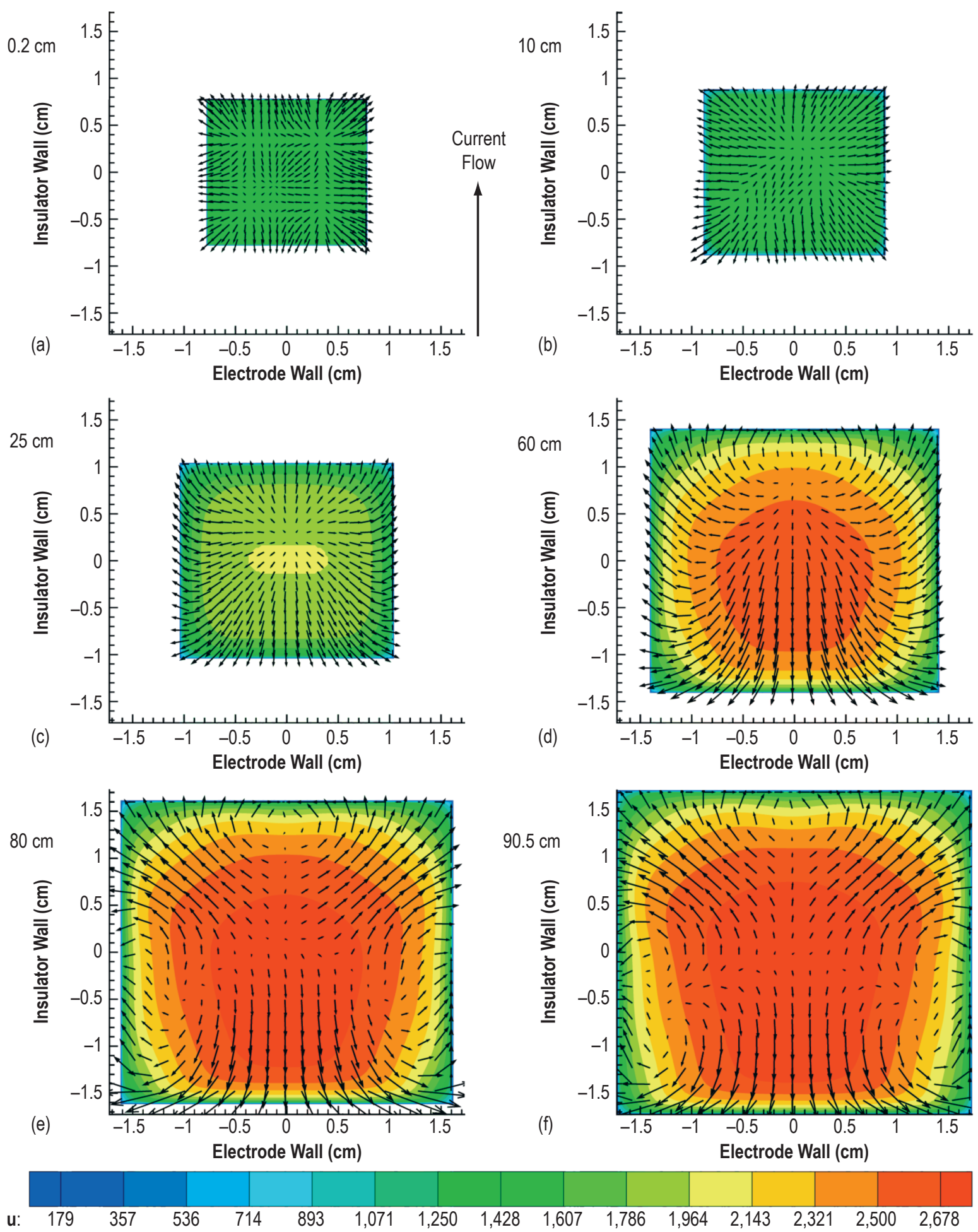

Figure 25. Vector plots of cross-sectional secondary flow velocities for (a) $0.2 \mathrm{~cm}$, (b) $10 \mathrm{~cm}$, (c) $25 \mathrm{~cm}$, (d) $60 \mathrm{~cm}$, (e) $80 \mathrm{~cm}$, and (f) $90.5 \mathrm{~cm}$ with the axial velocity, $\mathbf{u}$, shown as a background contour plot. Positive $J_{y}$ current flows from the bottom electrode to the top electrode. The length of the vectors is representative of their relative intensities. All velocities are in $\mathrm{m} / \mathrm{s}$. 


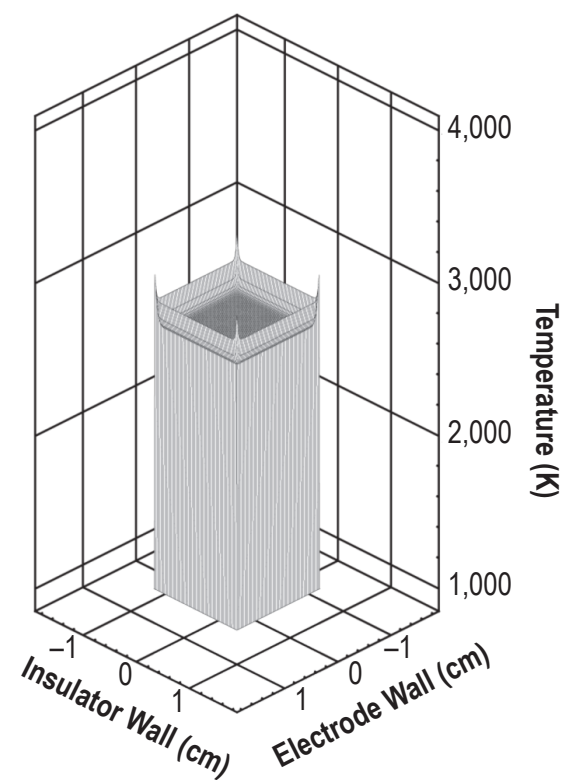

(a) $0.2 \mathrm{~cm}$

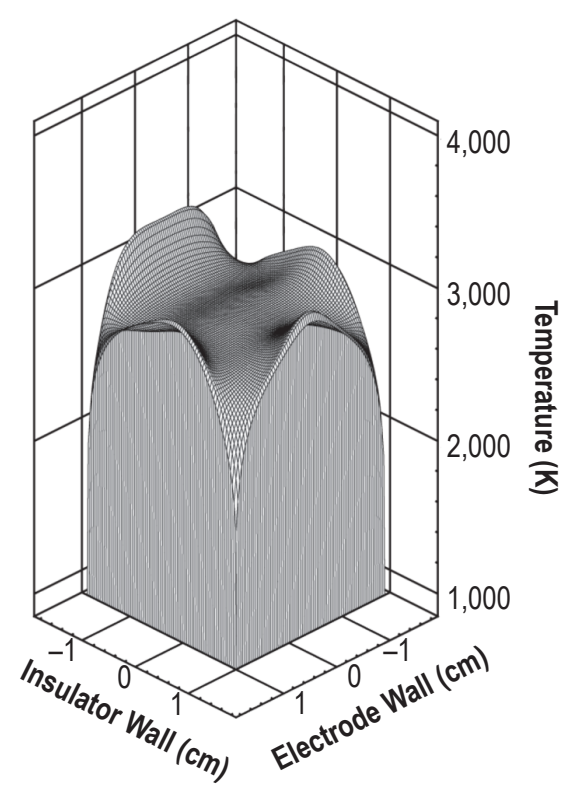

(d) $60 \mathrm{~cm}$

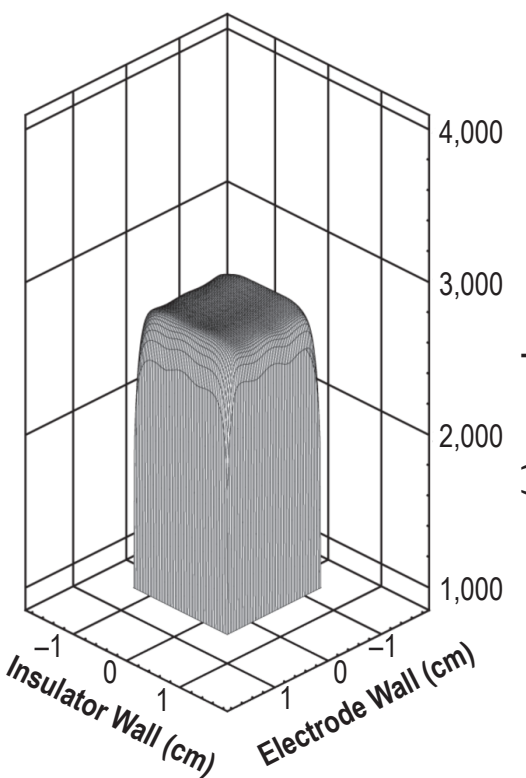

(b) $10 \mathrm{~cm}$

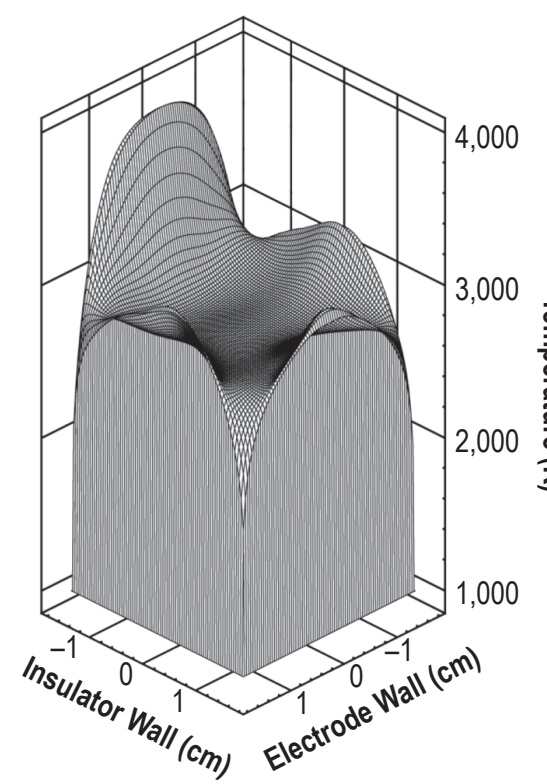

(e) $80 \mathrm{~cm}$

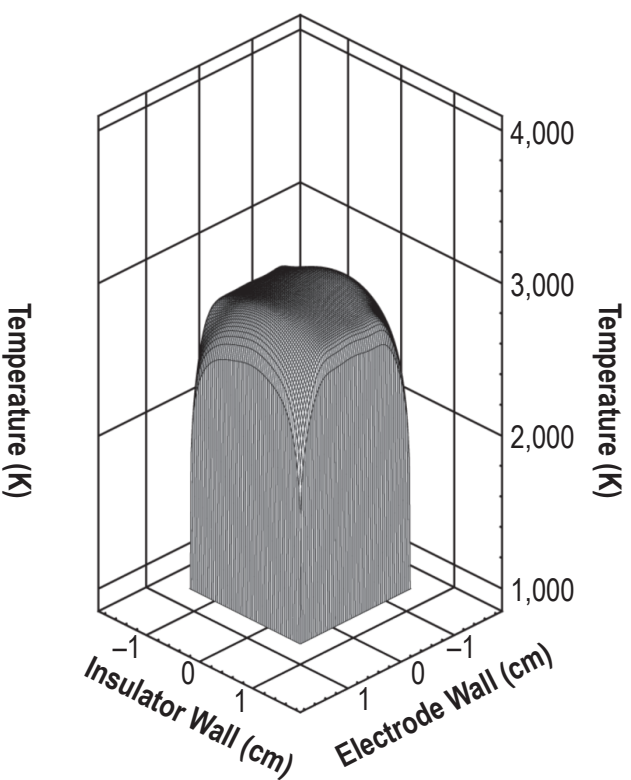

(c) $25 \mathrm{~cm}$

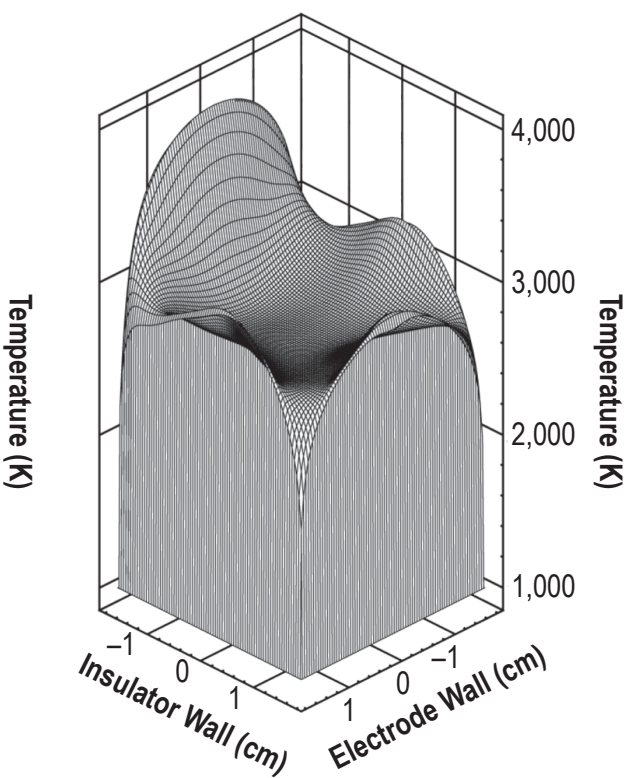

(f) $90.5 \mathrm{~cm}$

Figure 26. Three-dimensional temperature plots at various downstream locations in the MAPX accelerator channel: (a) $0.2 \mathrm{~cm}$, (b) $10 \mathrm{~cm}$, (c) $25 \mathrm{~cm}$, (d) $60 \mathrm{~cm}$, (e) $80 \mathrm{~cm}$, and (f) $90.5 \mathrm{~cm}$. Positive $J_{y}$ current density flows from the bottom electrode (background) to the top electrode (foreground) in accelerator mode. 


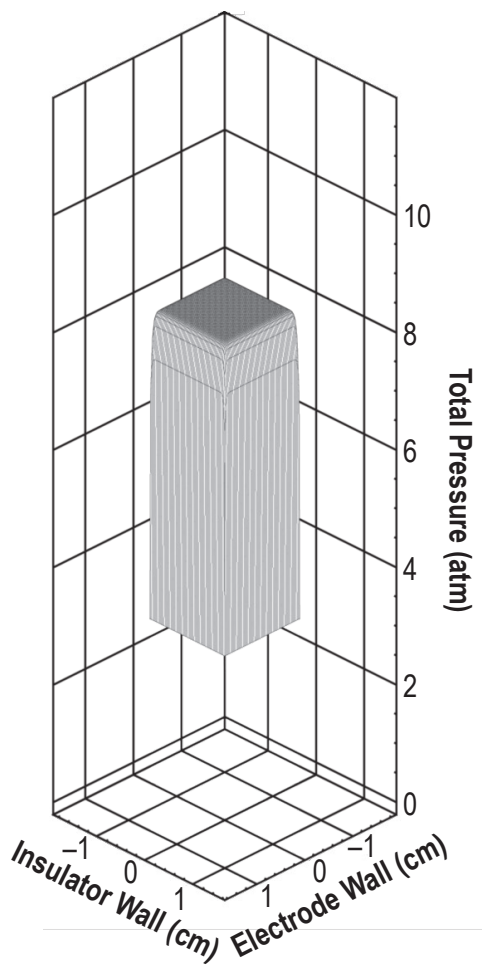

(a) $0.2 \mathrm{~cm}$

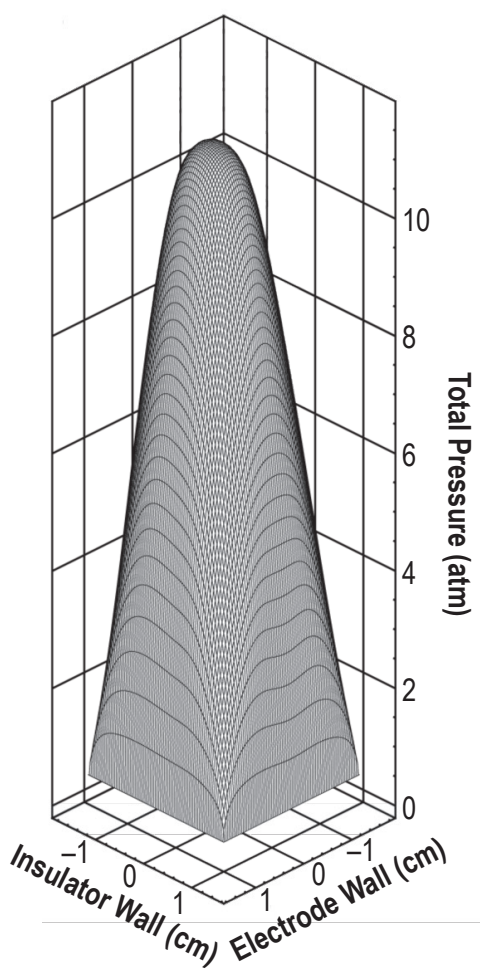

(d) $60 \mathrm{~cm}$

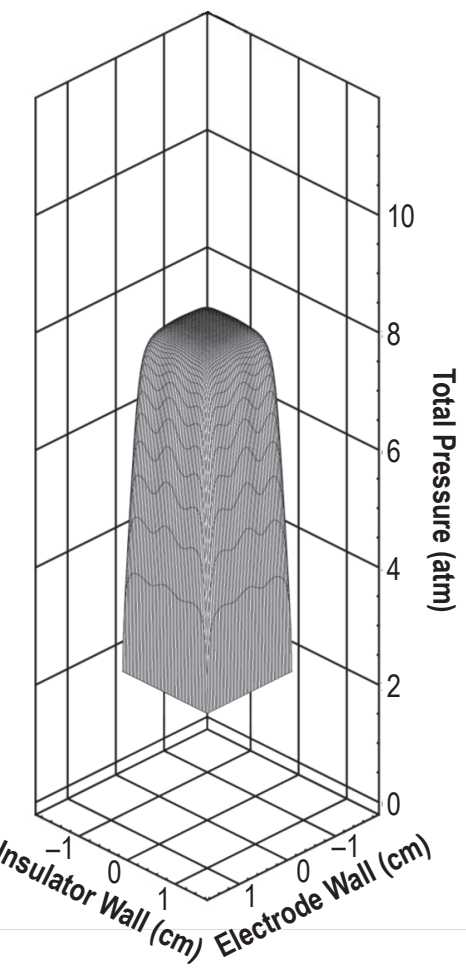

(b) $10 \mathrm{~cm}$

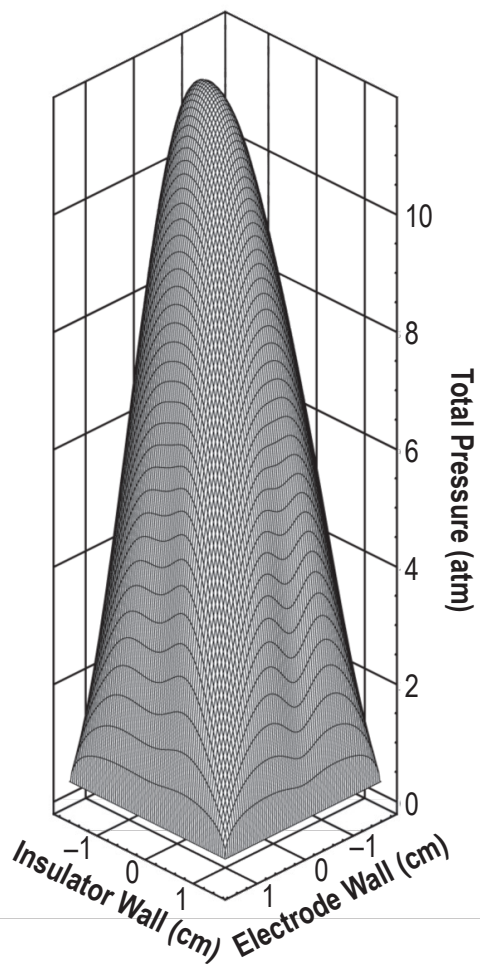

(e) $80 \mathrm{~cm}$

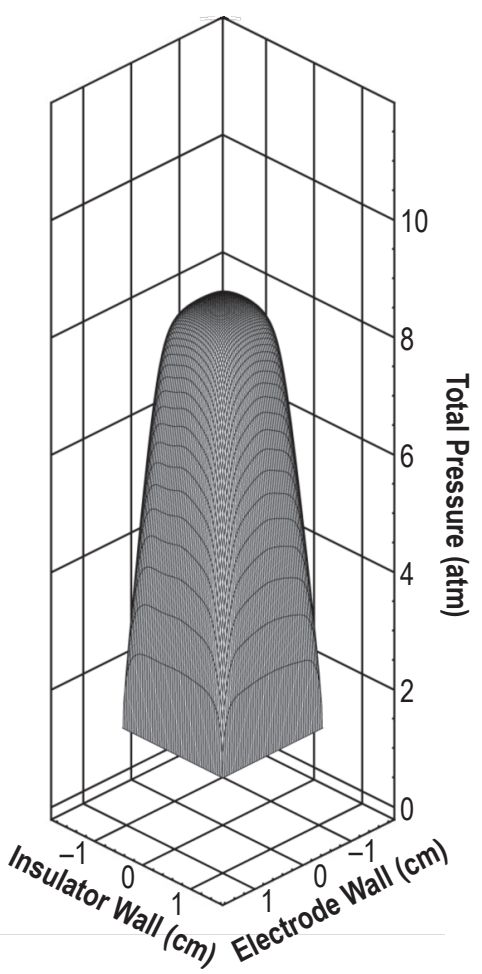

(c) $25 \mathrm{~cm}$

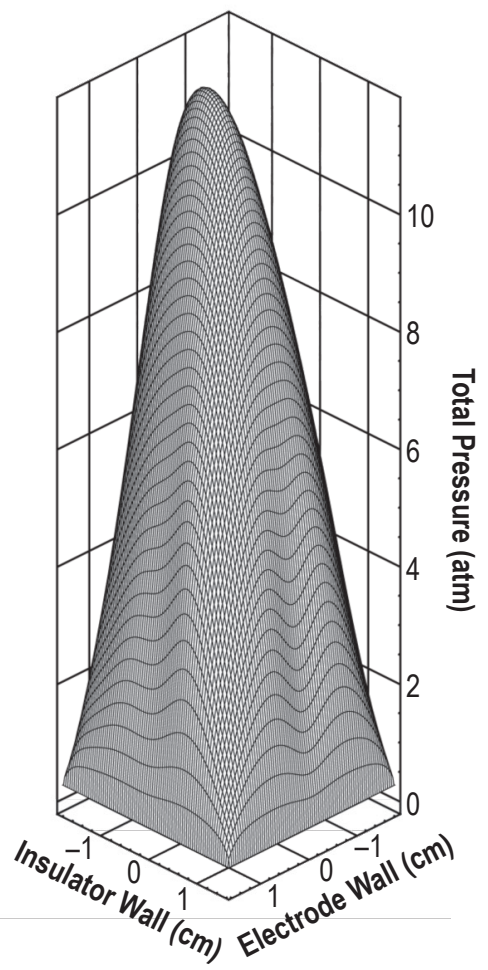

(f) $90.5 \mathrm{~cm}$

Figure 27. Three-dimensional total pressure plots at various downstream locations: (a) $0.2 \mathrm{~cm}$, (b) $10 \mathrm{~cm}$, (c) $25 \mathrm{~cm}$, (d) $60 \mathrm{~cm}$, (e) $80 \mathrm{~cm}$, and (f) $90.5 \mathrm{~cm}$. Positive $J_{y}$ current flow is from the background to the foreground. 
Figure 20 shows that the cross-sectional averaged transverse current density, $J_{y}$, declines along the accelerator channel, but never turns negative. Figure 28 reiterates that point with a contour plot of the transverse current density. The most intense current density occurs at $\approx 10 \mathrm{~cm}$. Beyond that axial location, the current density declines, and the lowest current densities occur near the center of the channel. Again, a portion of the decline in current density may be simply attributed to the increase in channel cross-sectional area.

Because $J_{y}$ and $J_{z}$ currents flow in the cross-sectional plane, they can be accurately visualized in relation to the secondary fluid flow. Figure 29 shows the total current flow in the cross-sectional plane, which is basically the vector sum of the $J_{y}$ and $J_{z}$ current densities. The most important aspect of the cross-sectional current is that it does not reverse direction and begin flowing in the negative direction. As long as $J_{y}$ is positive, the $J_{y} B$ Lorentz force still provides some push to the flow. If the current reverses direction to flow negative, then the Lorentz force would act to decelerate the flow.

In comparison to figure 29, figure 30 shows the vector plot of cross-sectional current densities for the 175-A applied current level. Here, the high applied current level yields a positive $J_{y}$ somewhat beyond the $40-\mathrm{cm}$ location. At the $60-\mathrm{cm}$ position, however, $J_{y}$ begins flowing in the negative direction and continues to do so through the $80-\mathrm{cm}$ location. This phenomenon sets up a decelerating force in opposition to the bulk flow direction.

One explanation for the current reversal depicted in figure 30 is the intense heat generation shown in figure 31. Starting as early as $45 \mathrm{~cm}$, the lower half of the channel begins to experience excessive heating. Between 70 and $90 \mathrm{~cm}$, an intense hot spot develops along the bottom electrode, much like the 100-A case of figure 26, but to a lesser degree. The intense heating at 175 A may also be seen in figure 18, thereby explaining the low efficiency, current reversal, and overall poor performance.

Figure 21 shows the centerline and cross-sectional averaged axial current density, $J_{x}$, as a function of axial location. The large differences between the centerline and cross-sectional averaged current densities in figure 21 indicate strong localized, opposing currents in the $x$-direction. Figure 32 confirms that the MAPX accelerator channel does have very intense opposing local axial currents in the crosssectional plane. At the $80-\mathrm{cm}$ position, for instance, very strong, local positive currents exist along the bottom electrode. 

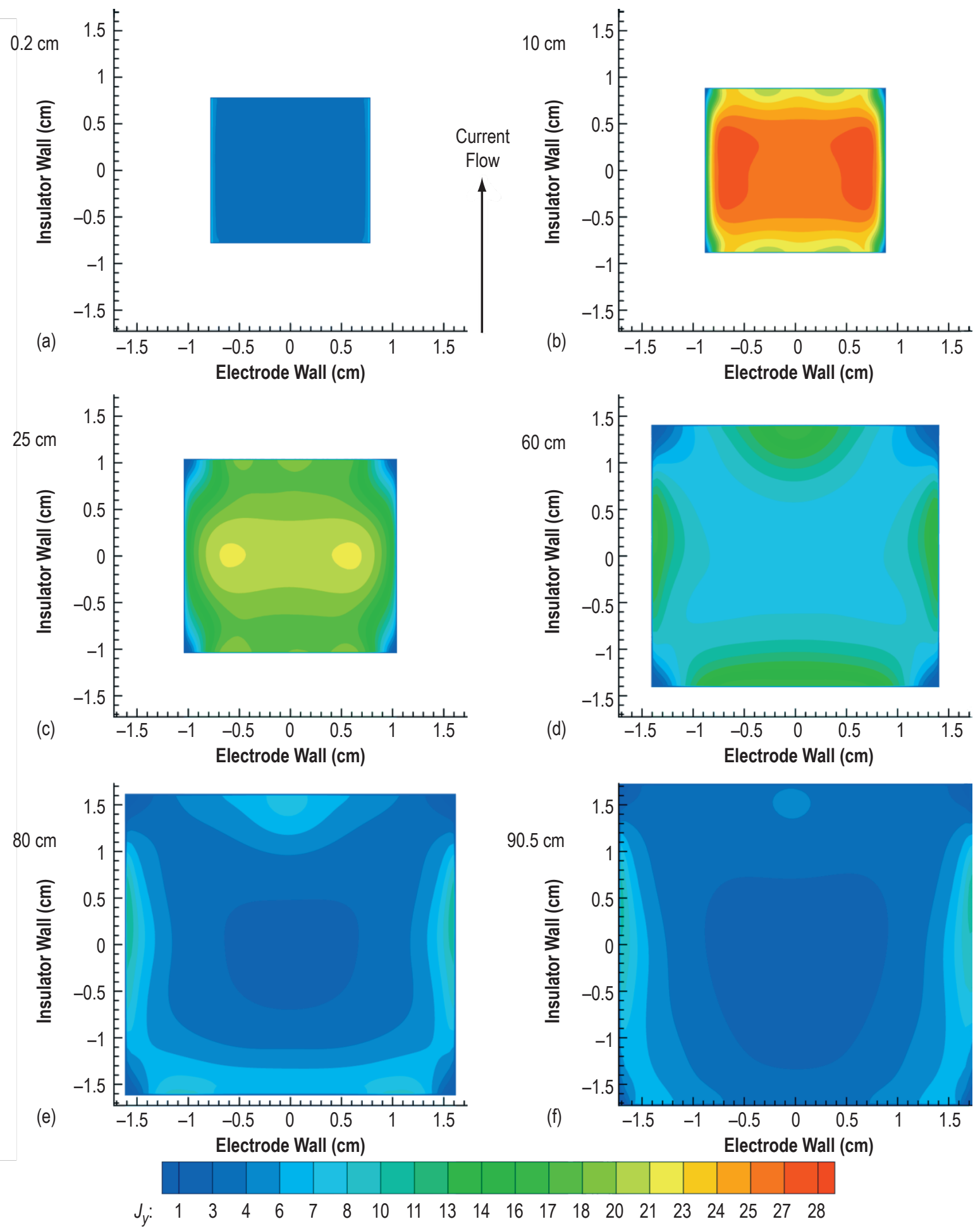

Figure 28. Contour plots of the $J_{y}$ current density $\left(\mathrm{A} / \mathrm{cm}^{2}\right)$ at various downstream locations: (a) $0.2 \mathrm{~cm}$, (b) $10 \mathrm{~cm}$, (c) $25 \mathrm{~cm}$, (d) $60 \mathrm{~cm}$, (e) $80 \mathrm{~cm}$, and (f) $90.5 \mathrm{~cm}$. Positive $J_{y}$ current flow goes from the bottom electrode to the top electrode. 

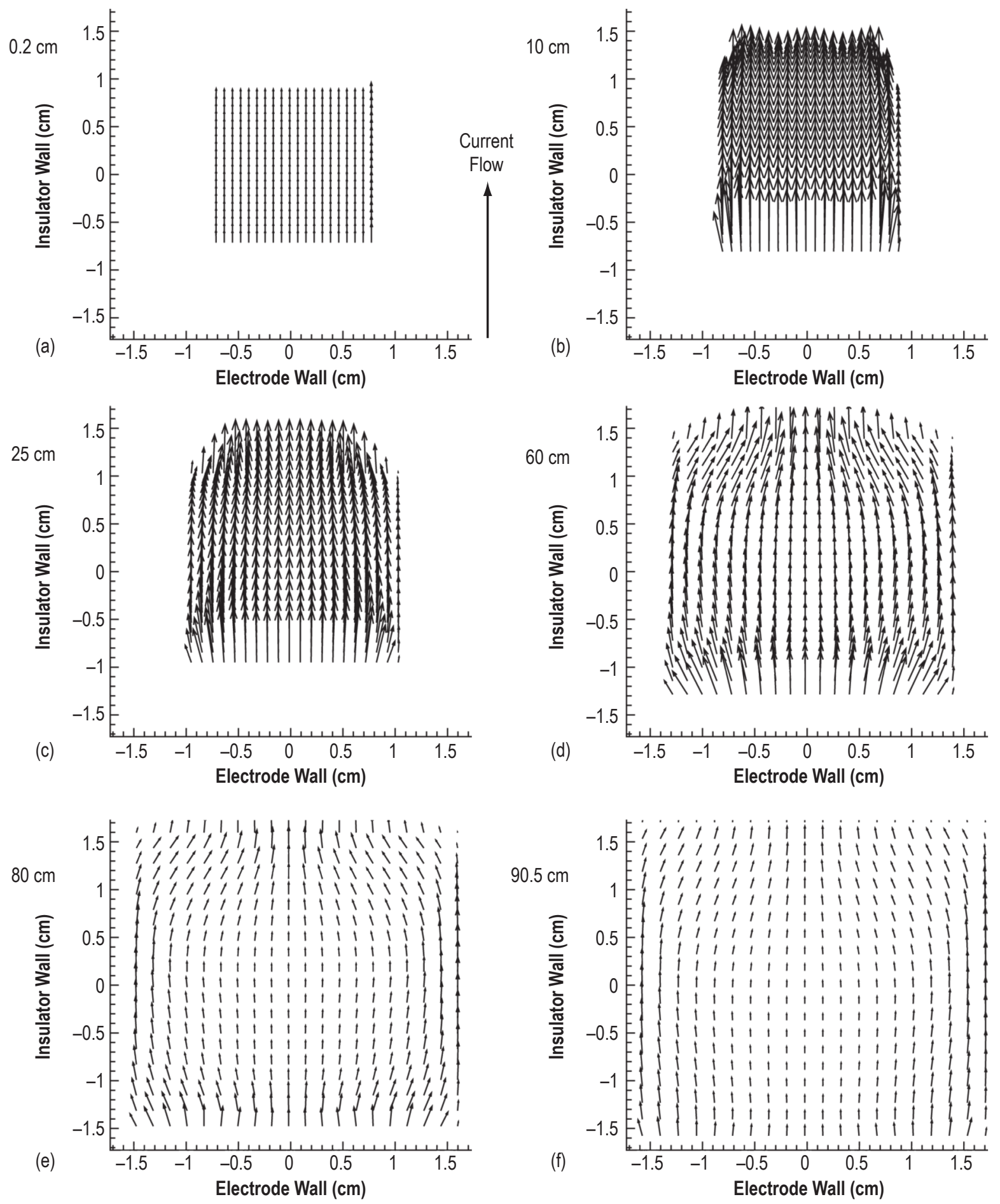

Figure 29. Vector plots of cross-sectional current densities $J_{y}$ and $J_{z}$ at various downstream locations in the MAPX accelerator channel for the 100-A case: (a) $0.2 \mathrm{~cm}$, (b) $10 \mathrm{~cm}$, (c) $25 \mathrm{~cm}$, (d) $60 \mathrm{~cm}$, (e) $80 \mathrm{~cm}$, and (f) $90.5 \mathrm{~cm}$. Positive $J_{y}$ current flow is from the bottom to top electrodes. 

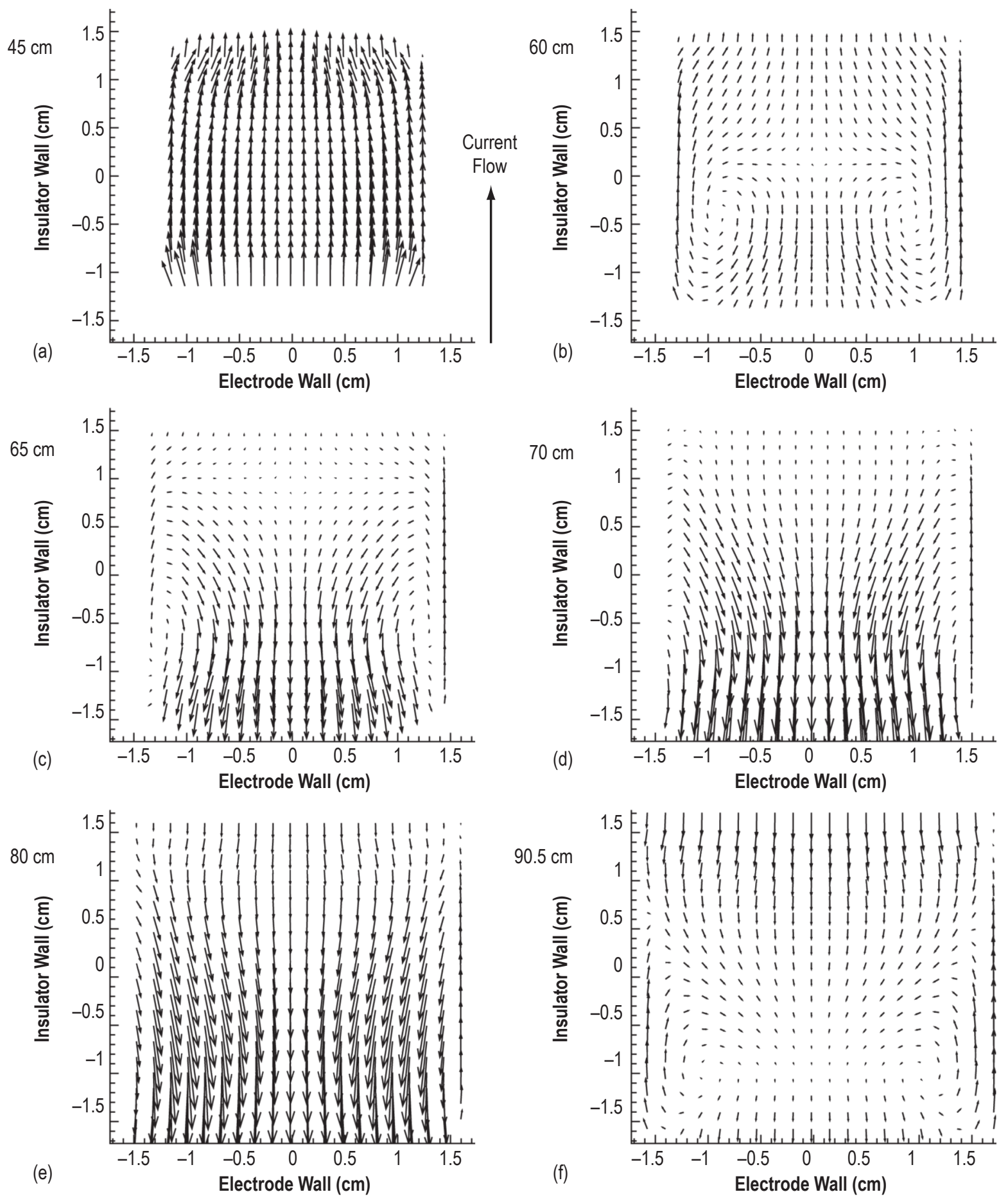

Figure 30. Vector plots of the cross-sectional current flow at various downstream locations for the 175-A case: (a) $45 \mathrm{~cm}$, (b) $60 \mathrm{~cm}$, (c) $65 \mathrm{~cm}$, (d) $70 \mathrm{~cm}$, (e) $80 \mathrm{~cm}$, and (f) $90.5 \mathrm{~cm}$. The positive $J_{y}$ current density is from the bottom to top electrode when in accelerator mode. 

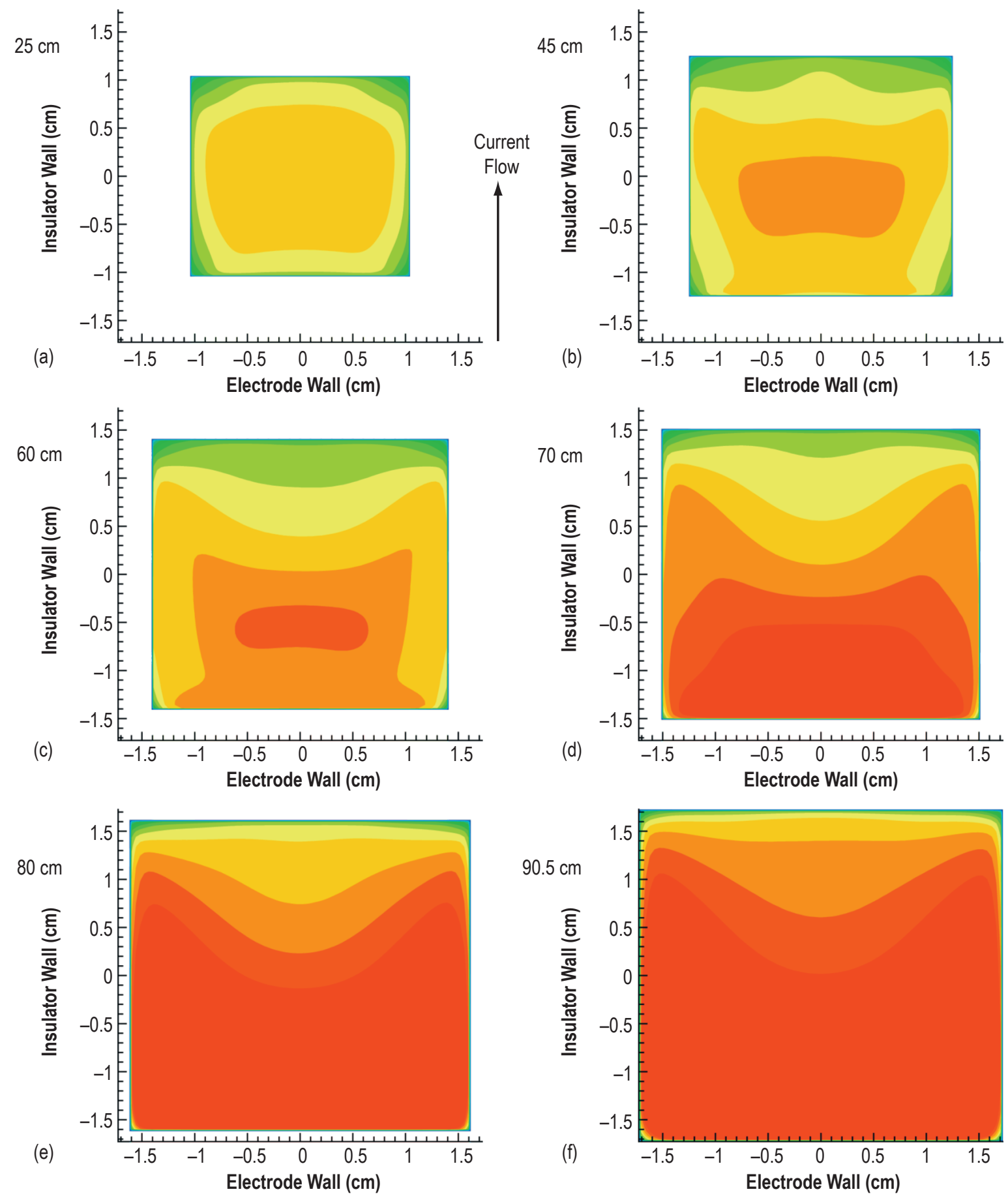

$T$ :

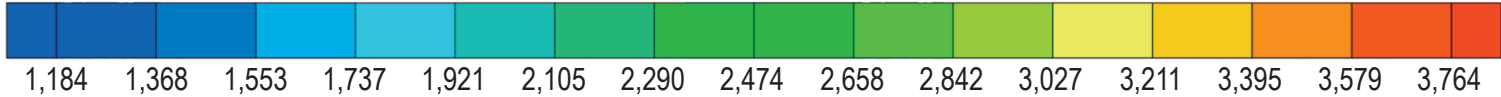

Figure 31. Contour plot of channel temperature $(\mathrm{K})$ at various downstream locations for the 175-A case: (a) $25 \mathrm{~cm}$, (b) $45 \mathrm{~cm}$, (c) $60 \mathrm{~cm}$, (d) $70 \mathrm{~cm}$, (e) $80 \mathrm{~cm}$, and (f) $90.5 \mathrm{~cm}$. Positive $J_{y}$ current flow occurs from the bottom to top electrode when in accelerator mode. 


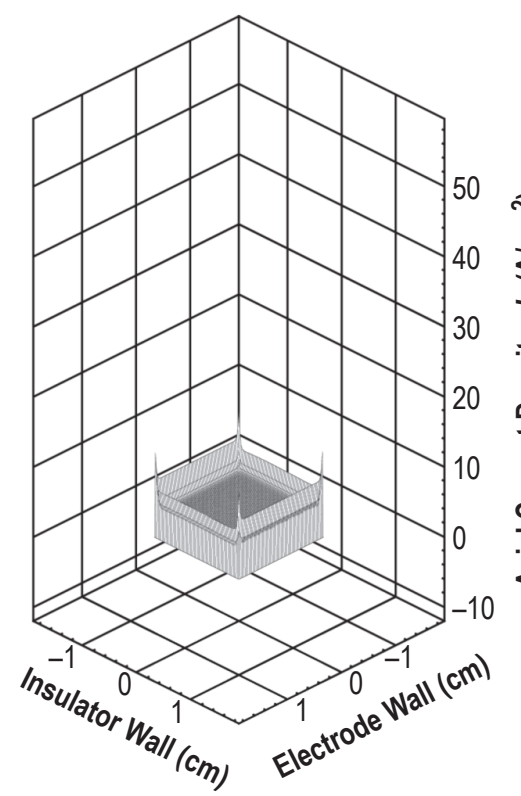

(a) $0.2 \mathrm{~cm}$

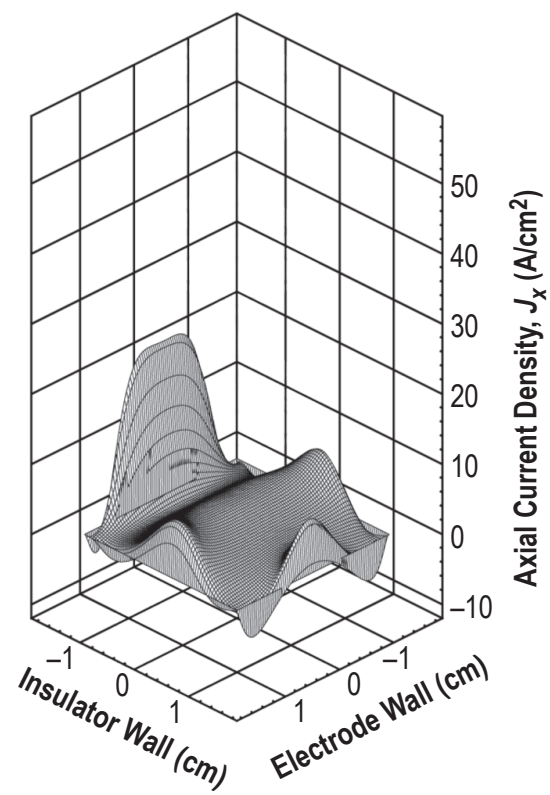

(d) $60 \mathrm{~cm}$

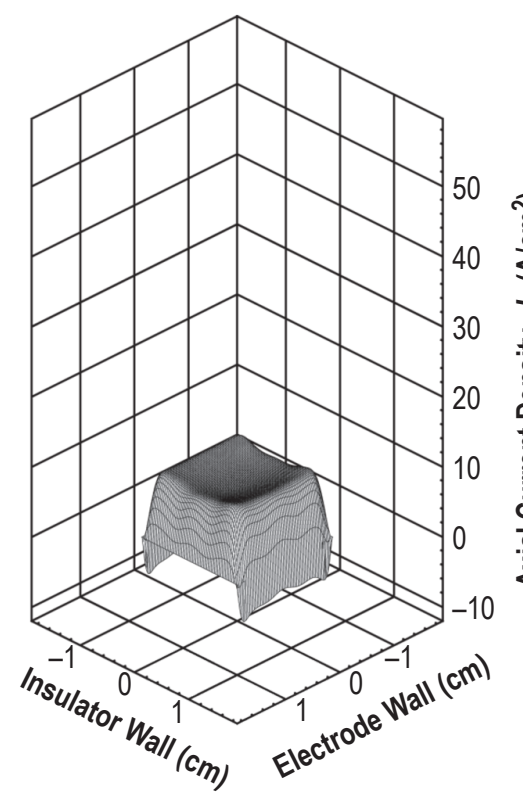

(b) $10 \mathrm{~cm}$

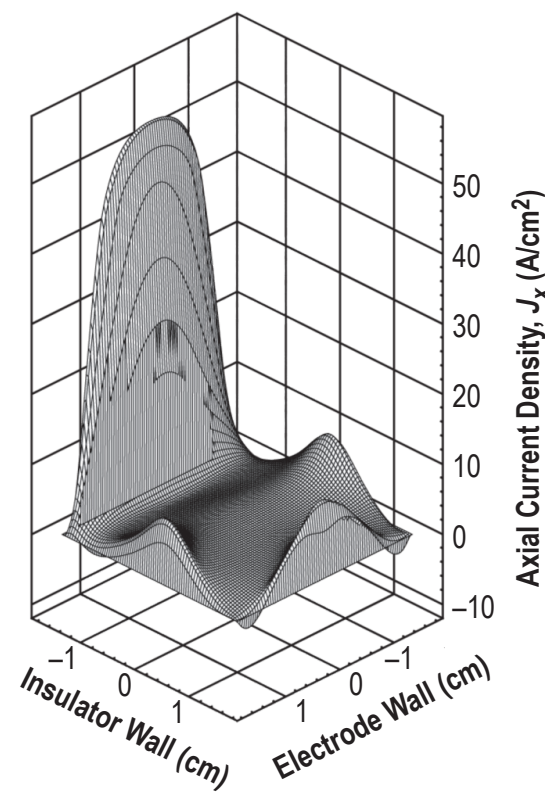

(e) $80 \mathrm{~cm}$

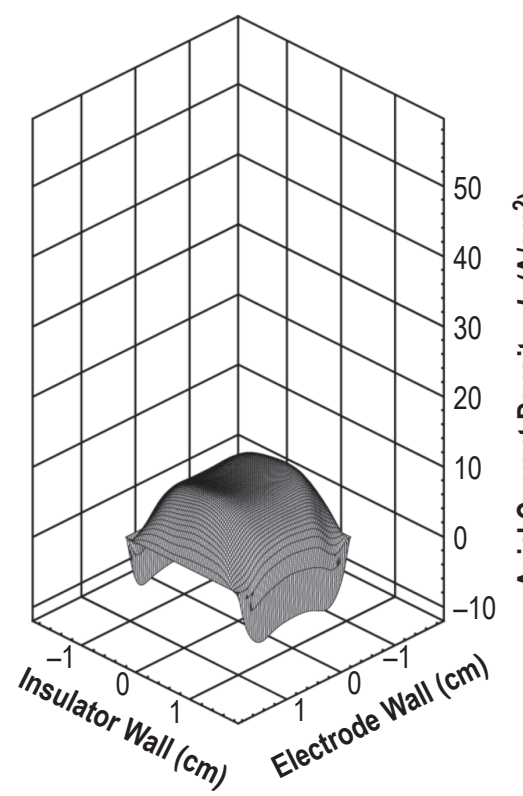

(c) $25 \mathrm{~cm}$

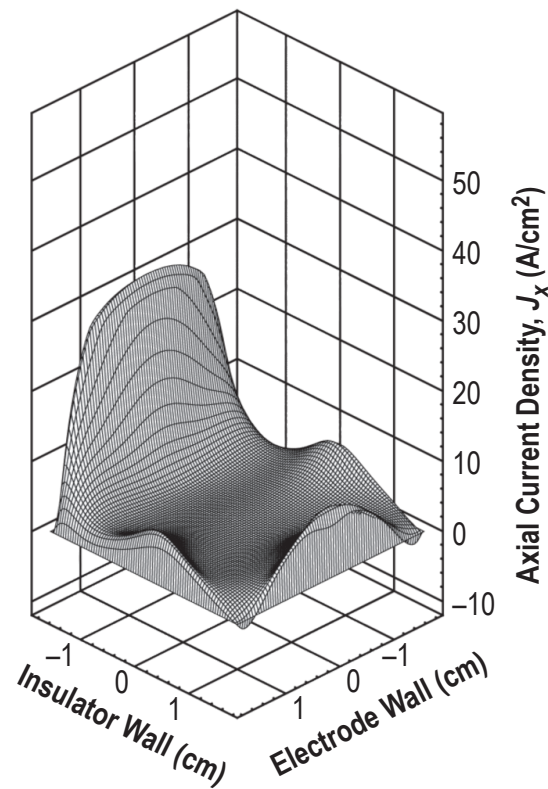

(f) $90.5 \mathrm{~cm}$

Figure 32. Three-dimensional axial current, $J_{x}$, plots at various downstream locations: (a) $0.2 \mathrm{~cm}$, (b) $10 \mathrm{~cm}$, (c) $25 \mathrm{~cm}$, (d) $60 \mathrm{~cm}$, (e) $80 \mathrm{~cm}$, and (f) $90.5 \mathrm{~cm}$. The positive $J_{y}$ current flows from the bottom electrode (background) to top electrode (foreground) in accelerator mode. 



\section{CONCLUSION}

A three-dimensional numerical MHD model was developed for the purpose of analyzing and optimizing the MAPX. The results of this analysis were intended to offer a better understanding of the critical physical processes within the MHD accelerator channel, to assist in the identification of an optimized configuration for initial testing, and to provide detailed pretest performance predictions of that optimized configuration.

The three-dimensional nature of the coupled fluid dynamic and electromagnetic effects in MHD flows, particularly enclosed channel flows, requires the use of a proper three-dimensional numerical model. Unfortunately, no such model was generally available for accelerator configurations at the beginning of the MAPX project. Therefore, in order to achieve the capabilities and accuracy required for a comprehensive analysis, modifications and additions were made to an existing three-dimensional PNS model previously developed by the Argonne National Laboratory for MHD generator channels. The resulting model, with integrated thermodynamic and electrical transport property routines established on the basis of a rigorous kinetic theory treatment with reliable collisional cross-section data, was then used to simulate the partially ionized flow inside the MAPX accelerator channel.

The results of an extensive three-dimensional analysis and trade study of the MAPX accelerator revealed critical effects affecting performance and provided assistance in the definition of an optimized configuration for initial testing. These results clearly indicate that entropy-producing mechanisms dominate the flow due to the high surface-to-volume ratio of the MAPX channel. More specifically, accelerator performance was found to be extremely sensitive to turbulent shear losses, which are difficult to simulate with high precision. Furthermore, large local axial currents cause asymmetries in the flow, resulting in localized runaway heating. The results from this extensive performance optimization study indicated that the MHD accelerator should utilize a $45^{\circ}$ diagonalization angle with the applied current evenly distributed over the first five inlet electrode pairs. When powered at $100 \mathrm{~A}$, this configuration is expected to yield a $50 \%$ global efficiency with an $80 \%$ increase in axial velocity and a $50 \%$ increase in centerline total pressure. 


\section{REFERENCES}

1. Litchford, R.J.; Cole, J.W.; Lineberry, J.T.; Chapman, J.N.; Schmidt, H.J.; and Lineberry, C.W.: "Magnetohydrodynamic Augmented Propulsion Experiment: Performance Analysis and Design," AIAA-2002-2184, 33rd Plasmadynamics and Lasers Conference/14th International Conference on MHD Power Generation and High Temperature Technologies, May 2002.

2. Litchford, R.J.; and Lineberry, J.T.: "Status of Magnetohydrodynamic Augmented Propulsion Experiment," AIAA-2007-3884, 38th Plasmadynamics and Lasers Conference/16th International Conference on MHD Energy Conversion, Miami, FL, June 25-28, 2007.

3. Bouillard, J.X.; Krazinski, J.L.; Vanka, S.P.; and Berry, G.F.: 'User's Manual for MGMHD: A Multi-Grid Three-Dimensional Computer Code for the Analysis of Magnetohydrodynamic Generators and Diffusers," ANL/MHD-89/1, Argonne National Laboratory, Argonne, IL, 1989.

4. McBride, J.; and Gordon, S.: "Computer Program for Calculation of Complex Chemical Equilibrium Compositions and Applications: II. Users Manual and Program Description," NASA RP-1311, NASA Lewis Research Center, Cleveland, OH, June 1996.

5. Devoto, R.S.: “The Transport Properties of a Partially Ionized Monatomic Gas," Ph.D. Dissertation, UMI: 65-9464, Stanford University, Stanford, CA, 1965.

6. Wu, Y.C.L.: "Performance Theory of Diagonal Conducting Wall MHD Generators," AIAA Journal, Vol. 14, No. 10, pp. 1362-1368, October 1976.

7. Litchford, R.J.: "Performance Theory of Diagonal Conducting Wall MHD Accelerators," AIAA Journal of Propulsion \& Power, Vol. 20, No. 4, pp. 742-750, July-August 2004.

8. Bityurin, V.A.; Zatelepin, V.N.; and Lyubimov, G.A.: "A Consideration of Some Three-Dimensional Effects in MHD Channels," Proceedings of 16th Symposium on Engineering Aspects of Magnetohydrodynamics (SEAM), Pittsburgh, PA, May 1977.

9. Ahluwalia, R.K.; Vanka, S.P.; Im, K.H.; and Zwick, S.A.: "Formulation and Assessment of a Cross-Plane Electrical Model for Magnetohydrodynamic Channels," Journal of Energy, Vol. 6, No. 5, pp. 314-322, September-October 1982.

10. Turner, M.W.: "Three-Dimensional Numerical Modeling of a Diagonal Magnetohydrodynamic Accelerator," Ph.D. Dissertation, The University of Alabama in Huntsville, Huntsville, AL, 2007.

11. Spencer, F.E.; and Phelps, A.V.: "Momentum Transfer Cross-Sections and Conductivity Integrals for Gases of MHD Interest," 15th Symposium of the Engineering Aspects of Magnetohydrodynamics (SEAM), University of Pennsylvania, Philadelphia, PA, 1976. 
12. Phelps, A.V.: JILA Collision Data Compilation, < $<$ http://jila.colorado.edu/collision_data/>, Accessed on June 10, 2004.

13. Hirschfelder, J.O.; Curtis, C.F.; and Bird, R.B.: Molecular Theory of Gases and Liquids, John Wiley and Sons, New York, 2nd Edition, 1964.

14. Chapman, S.; and Cowling, T.G.: The Mathematical Theory of Non-Uniform Gases, Cambridge University Press, Cambridge, 1970.

15. Mitchner, M.; and Kruger, C.H.: Partially Ionized Gases, John Wiley and Sons, New York, 1973.

16. Devoto, R.S.: "Simplified Expressions for the Transport of Properties of Ionized Monatomic Gases," The Physics of Fluids, Vol. 10, No. 2, pp. 354-364, October 17, 1967.

17. Zollweg, R.J.; and Liebermann, R.W.: "Electrical Conductivity of Nonideal Plasmas," Journal of Applied Physics, Vol. 62, No. 6, pp. 3621-3627, November 1, 1987.

18. Vanka, S.P.; and Ahluwalia, R.K.: "Three-Dimensional Flow and Thermal Development in Magnetohydrodynamic Channels," Journal of Energy, Vol. 6, No. 3, pp. 218-224, May-June 1982. 


\section{REPORT DOCUMENTATION PAGE}

The public reporting burden for this collection of information is estimated to average 1 hour per response, including the time for reviewing instructions, searching existing data sources, gathering and maintaining the data needed, and completing and reviewing the collection of information. Send comments regarding this burden estimate or any other aspect of this collection of information, including suggestions for reducing this burden, to Department of Defense, Washington Headquarters Services, Directorate for Information Operation and Reports (0704-0188), 1215 Jefferson Davis Highway, Suite 1204, Arlington, VA 22202-4302. Respondents should be aware that notwithstanding any other provision of law, no person shall be subject to any penalty for failing to comply with a collection of information if it does not display a currently valid OMB control number

PLEASE DO NOT RETURN YOUR FORM TO THE ABOVE ADDRESS.
1. REPORT DATE (DD-MM-YYYY)
2. REPORT TYPE

01-10-2009

Technical Memorandum

4. TITLE AND SUBTITLE

Three-Dimensional Numerical Modeling of Magneto-

hydrodynamic Augmented Propulsion Experiment

6. AUTHOR(S)

5d. PROJECT NUMBER

M.W. Turner,* C.W. Hawk,* and R.J. Litchford

5e. TASK NUMBER

5f. WORK UNIT NUMBER

7. PERFORMING ORGANIZATION NAME(S) AND ADDRESS(ES)

George C. Marshall Space Flight Center

Marshall Space Flight Center, AL 35812

8. PERFORMING ORGANIZATION

REPORT NUMBER

9. SPONSORING/MONITORING AGENCY NAME(S) AND ADDRESS(ES)

$\mathrm{M}-1268$

National Aeronautics and Space Administration

Washington, DC 20546-0001

NASA/TM-2009-216113

12. DISTRIBUTION/AVAILABILITY STATEMENT

Unclassified-Unlimited

Subject Category 20

Availability: NASA CASI (443-757-5802)

13. SUPPLEMENTARY NOTES

*The University of Alabama in Huntsville, Huntsville, AL 35899

Prepared for the Propulsion Systems Department, Engineering Directorate

14. ABSTRACT

Over the past several years, NASA Marshall Space Flight Center has engaged in the design and development of an experimental research facility to investigate the use of diagonalized crossed-field magnetohydrodynamic (MHD) accelerators as a possible thrust augmentation device for thermal propulsion systems. In support of this effort, a three-dimensional numerical MHD model has been developed for the purpose of analyzing and optimizing accelerator performance and to aid in understanding critical underlying physical processes and nonideal effects. This Technical Memorandum fully summarizes model development efforts and presents the results of pretest performance optimization analyses. These results indicate that the MHD accelerator should utilize a $45^{\circ}$ diagonalization angle with the applied current evenly distributed over the first five inlet electrode pairs. When powered at $100 \mathrm{~A}$, this configuration is expected to yield a $50 \%$ global efficiency with an $80 \%$ increase in axial velocity and a $50 \%$ increase in centerline total pressure.

15. SUBJECT TERMS

propulsion, electric thruster, magnetohydrodynamics, plasmadynamics, computational fluid dynamics

\begin{tabular}{|l|c|c|c|c|}
\hline \multicolumn{2}{|l|}{ 16. SECURITY CLASSIFICATION OF: } & 17. LIMITATION OF ABSTRACT & $\begin{array}{c}\text { 18. NUMBER OF } \\
\text { PAGES }\end{array}$ \\
\cline { 1 - 3 } a. REPORT & b. ABSTRACT & c. THIS PAGE & & 68 \\
& $\mathrm{U}$ & $\mathrm{U}$ & $\mathrm{UU}$ & 6 \\
\end{tabular}

19a. NAME OF RESPONSIBLE PERSON STI Help Desk at email: help@sti.nasa.gov 19b. TELEPHONE NUMBER (Include area code) STI Help Desk at: 443-757-5802 

National Aeronautics and

Space Administration

IS20

George C. Marshall Space Flight Center

Marshall Space Flight Center, Alabama

35812 